\title{
Relations among partitions
}

\author{
R. A. Bailey
}

\begin{abstract}
Combinatorialists often consider a balanced incomplete-block design to consist of a set of points, a set of blocks, and an incidence relation between them which satisfies certain conditions. To a statistician, such a design is a set of experimental units with two partitions, one into blocks and the other into treatments; it is the relation between these two partitions which gives the design its properties. The most common binary relations between partitions that occur in statistics are refinement, orthogonality and balance. When there are more than two partitions, the binary relations may not suffice to give all the properties of the system. I shall survey work in this area, including designs such as double Youden rectangles.
\end{abstract}

\section{Introduction}

Many combinatorialists think of a balanced incomplete-block design (BIBD) as a set $\mathcal{P}$ of points together with a collection $\mathcal{B}$ of subsets of $\mathcal{P}$, called blocks, which satisfy various conditions. For example, see [52]. Some papers, such as $[16,65,199]$, call a BIBD simply a design. Others think of it as the pair of sets $\mathcal{P}$ and $\mathcal{B}$ with a binary incidence relation between their elements. These views are both rather different from that of a statistician who is involved in designing experiments. The following examples introduce the statistical point of view, as well as serving as a basis for the combinatorial ideas in this paper.

Example 1.1 A horticultural enthusiast wants to compare three varieties of lettuce for people to grow in their own gardens. He enlists twelve people in his neighbourhood. Each of these prepares three patches in their vegetable garden, and grows one of the lettuce varieties on each patch, so that each gardener grows all three varieties.

Here the patches of land are experimental units. There may be some differences between the gardeners, so the three patches in a single garden form what is called a block. Each variety occurs just once in each block, and so the blocks are said to be complete. Complete-block designs were advocated by Fisher in [78], and are frequently used in practice.

Example 1.2 Now suppose that the number of lettuce varieties is increased to nine. It is not reasonable to expect an amateur gardener to 


\begin{tabular}{|c|c|c|c|c|}
\hline$A$ & $C$ & $A$ & $B$ & $C$ \\
\hline$B$ & $D$ & $F$ & $D$ & $E$ \\
\hline$C$ & $H$ & $H$ & $I$ & $G$ \\
\hline
\end{tabular}

Figure 1: Balanced incomplete-block design in Example 1.2: columns represent blocks and letters represent varieties

grow nine different varieties, so each gardener still uses only three patches of ground, and thus can grow only three varieties. The blocks are now incomplete, in the terminology of Yates [225].

One possible layout is shown in Figure 1. This incomplete-block design has the property that each pair of distinct varieties concur in the same number of blocks (here, exactly one). Yates originally called incompleteblock designs with this property symmetrical, but the adjective had been changed to balanced within a few years $[46,80]$.

To a statistician, the partition of the set of experimental units into blocks is inherent and is known before the decision is taken about which variety to allocate to each unit. This allocation gives another partition of the set of experimental units, and it is the relation between these two partitions that is regarded as balance. It is not a symmetric relation, in general. In Example 1.2 the varieties are balanced with respect to the blocks, but the blocks are not balanced with respect to the varieties because some pairs of blocks have one variety in common while others have none. This relation is discussed in more detail in Section 5.

In fact, statisticians usually call these partitions factors, because the names of the parts are relevant. In Example 1.2 the names of the varieties are not interchangeable; we probably want to find out which one does best. Thus a factor is typically regarded as a function from the set of experimental units to a finite set: if $B$ and $L$ denote the factors for blocks and lettuce varieties respectively and $\omega$ is a vegetable patch then $B(\omega)$ is the block (garden) containing $\omega$ and $L(\omega)$ is the variety grown on $\omega$. Furthermore, $|B(\omega)|$ is the size of the block containing $\omega$, while $|L(\omega)|$ is the number of patches with the same variety as that grown on $\omega$.

A response $Y_{\omega}$, such as total yield of edible lettuce in kilograms, is measured on each patch $\omega$. It is usually assumed that $Y_{\omega}$ is a random variable and that there are constants $\tau_{i}$ and $\beta_{j}$ such that

$$
Y_{\omega}=\tau_{L(\omega)}+\beta_{B(\omega)}+\varepsilon_{\omega}
$$

where the final terms $\varepsilon_{\omega}$ are independent random variables with zero mean and the same variance $\sigma^{2}$; often they are assumed to be normally dis- 

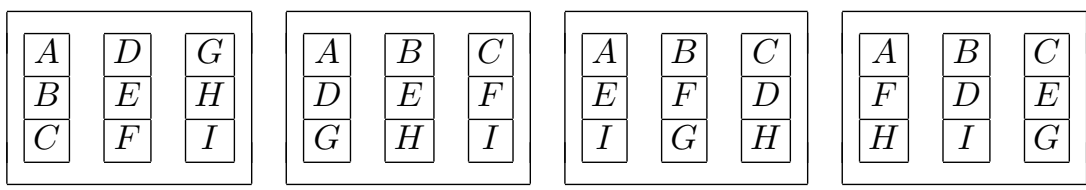

Figure 2: Resolved balanced incomplete-block design in Example 1.3: columns represent blocks, rectangles represent districts and letters represent varieties

tributed. The purpose of the experiment is to estimate the constants $\tau_{i}$. Of course, this is impossible, because equation (1.1) is unchanged if a constant is added to every $\tau_{i}$ and subtracted from every $\beta_{j}$, but we aim to estimate differences such as $\tau_{1}-\tau_{2}$, that is, to estimate the $\tau_{i}$ up to an additive constant.

Thus the two partitions have different roles. One (the partition $B$ ) is inherent, and we are usually not interested in the effects $\beta_{j}$ of the different parts. The other (the partition $L$ ) has its parts allocated by the experimenter, and the purpose of the experiment is to find out what differences there are between its parts. Nonetheless, this paper will concentrate on the combinatorial relation between them. Before doing so, we give some examples with three partitions.

Example 1.3 Suppose that the twelve gardeners in Example 1.2 do not all live in the same neighbourhood. Instead, they are spread over four different districts, with three per district. If the first three blocks in Figure 1 represent the gardens in the first district, and so on, then each variety is grown once in each district, as shown in Figure 2. This is convenient if other people want to look at the different varieties during the course of the experiment.

Each block is contained within a single district, so the partition into blocks is a refinement of the partition into districts. Section 3 discusses refinement in more detail. On the other hand, the partitions into districts and into varieties have the property that each part of one (a district) meets each part of the other (a variety) in a single experimental unit. This is a special case of strict orthogonality, which is explained in Section 4.

The assumption about $Y_{\omega}$ might remain as in (1.1) or it might be

$$
Y_{\omega}=\tau_{L(\omega)}+\beta_{B(\omega)}+\gamma_{D(\omega)}+\varepsilon_{\omega},
$$

where $D(\omega)$ is the district containing $\omega$. Of course, if the $\beta_{j}$ and $\gamma_{k}$ are all constants then they are not estimable, because we can add a constant 


\begin{tabular}{|c|c|c|c|c|c|c|}
\hline$A$ & $B$ & $C$ & $D$ & $E$ & $F$ & $G$ \\
\hline$C$ & $D$ & $E$ & $F$ & $G$ & $A$ & $B$ \\
\hline$D$ & $E$ & $F$ & $G$ & $A$ & $B$ & $C$ \\
\hline$E$ & $F$ & $G$ & $A$ & $B$ & $C$ & $D$ \\
\hline
\end{tabular}

Figure 3: Row-column design in Example 1.4: rows represent months, columns represent people and letters represent exercise regimes

to $\gamma_{1}$ and subtract it from $\beta_{j}$ for all blocks $j$ in district 1 . However, it is sometimes assumed that the $\beta_{j}$ are independent random variables with zero mean and the same variance $\sigma_{B}^{2}$. Section 6.2 discusses further the potential difficulty in an assumption like (1.2) when one partition is a refinement of another.

An incomplete-block design whose blocks can be grouped into collections each of which contains each variety just once, as in Example 1.3, is called resolvable. Section 15 gives more information about such designs.

Example 1.4 In order to assess the benefits of different exercise regimes, a health scientist asks seven healthy people to participate in an experiment over four months. Each month each person will be allocated one of seven exercise regimes. At the end of each month, the change in some measure of fitness, such as heart rate, will be recorded for each person.

Now each experimental unit is one person for one month. The partitions into months and into people are inherent, but the scientist chooses the partition into exercise regimes. Figure 3 shows one possible design for this experiment. The partitions into months and into people are strictly orthogonal to each other, as are the partitions into months and into exercise regimes. The partitions into people and into exercise regimes are both balanced with respect to each other.

Example 1.5 A small modification of Example 1.4 has five months, six people and ten exercise regimes. One possible design is shown in Figure 4, where rows represent months, columns respresent people and letters represent exercise regimes.

Example 1.6 A modification of Example 1.2 has ten gardens of three vegetable patches each, and six varieties of lettuce. In addition, there are are five possible watering regimes. Each patch must have one variety of lettuce and one watering regime. The design in Figure 4 can be used, but now rows represent watering regimes, columns represent lettuce varieties and letters represent gardens. 


\begin{tabular}{|c|c|c|c|c|c|}
\hline$H$ & $J$ & $I$ & $G$ & $F$ & $E$ \\
\hline$J$ & $I$ & $H$ & $C$ & $B$ & $D$ \\
\hline$D$ & $F$ & $A$ & $J$ & $G$ & $C$ \\
\hline$A$ & $B$ & $G$ & $E$ & $D$ & $I$ \\
\hline$E$ & $A$ & $C$ & $B$ & $H$ & $F$ \\
\hline
\end{tabular}

Figure 4: Combinatorial design used in Examples 1.5 and 1.6

Denote by $R, C$ and $L$ the partitions into rows, columns and letters in the design in Figure 4. From the point of view of the statistician, the uses of this design in Examples 1.5 and 1.6 are quite different. In the former, the partitions $R$ and $C$ are inherent while $L$ is at the choice of the experimenter; in the latter, $L$ is inherent while the experimenter chooses $R$ and $C$. However, in both cases it may be assumed that

$$
Y_{\omega}=\alpha_{R(\omega)}+\phi_{C(\omega)}+\tau_{L(\omega)}+\varepsilon_{\omega} .
$$

From a combinatorial point of view, Figure 4 simply shows a set with three partitions. The partitions $R$ and $C$ are strictly orthogonal to each other, while each of $R$ and $C$ is balanced with respect to letters. In fact, there is a third property, called adjusted orthogonality, that will be defined in Section 8.

For further explanation of how combinatorial design problems arise from statistically designed experiments, see [22, 33, 177, 202].

The remainder of this paper treats a combinatorial design as a collection of partitions of a finite set. Section 2 establishes some notation for partitions and their associated matrices and subspaces. Sections 3-5 discuss the three most important binary relations between partitions, all of which have been seen in the examples so far. Section 6 explains more about the background to equations (1.1)-(1.3). Section 7 discusses the relations between the subspaces defined by partitions, and shows that sometimes there is a need for a ternary relation. Sections 8 and 9 give more details of two important non-binary relations. These are used in Section 10, which considers possibilities for three partitions. This leads to several different types of combinatorial design, considered in the remaining sections. Each type is defined by three partitions, or is a simple generalization with more partitions but no need for any further non-binary relations.

\section{Partitions on a finite set}

Let $\Omega$ be a finite set of size $e$, where $e>1$. The elements of $\Omega$ will be called experimental units, or just units. The rest of this paper deals with 
partitions of $\Omega$.

If $F$ is such a partition, denote by $n_{F}$ its number of parts. The $e \times n_{F}$ incidence matrix $X_{F}$ has $(\omega, i)$-entry equal to 1 if unit $\omega$ is in part $i$ of $F$; otherwise, this entry is zero. Thus $X_{F} X_{F}^{\top}$ is the $e \times e$ relation matrix for $F$, with $\left(\omega_{1}, \omega_{2}\right)$-entry equal to 1 if $\omega_{1}$ and $\omega_{2}$ are in the same part of $F$, and equal to 0 otherwise. The $n_{F} \times n_{F}$ matrix $X_{F}^{\top} X_{F}$ is diagonal, with $(i, i)$-entry equal to the size of the $i$-th part of $F$.

Definition A partition is uniform if all of its parts have the same size.

Many statisticians, including Tjur [206, 207], call uniform partitions balanced, but this conflicts with the notion of balance introduced in Section 1. This terminology is discussed again in Section 9. Preece reviewed the overuse of the word balance in design of experiments in [155]. The adjectives homogeneous [44], proper [151] and regular [66] are also used.

If $F$ is uniform, denote the size of all its parts by $k_{F}$. Then $n_{F} k_{F}=e$ and $X_{F}^{\top} X_{F}=k_{F} I_{n_{F}}$, where $I_{n}$ is the identity matrix of order $n$.

Denote by $\mathbf{R}^{\Omega}$ the real vector space of dimension $e$ whose coordinates are labelled by the elements of $\Omega$, so that each vector may be regarded as a function from $\Omega$ to $\mathbf{R}$. If $F$ is a partition of $\Omega$, denote by $V_{F}$ the subspace of $\mathbf{R}^{\Omega}$ consisting of vectors which are constant on each part of $F$. Then $\operatorname{dim}\left(V_{F}\right)=n_{F}$.

We assume the standard inner product on $\mathbf{R}^{\Omega}$. Denote by $P_{F}$ the matrix of orthogonal projection onto $V_{F}$. Then $P_{F}$ replaces the coordinate $y_{\omega}$ of any vector $y$ by the average value of $y_{\nu}$ for $\nu$ in $F(\omega)$, which is the part of $F$ containing $\omega$. In fact, $P_{F}=X_{F}\left(X_{F}^{\top} X_{F}\right)^{-1} X_{F}^{\top}$. If $F$ is uniform then $X_{F} X_{F}^{\top}=k_{F} P_{F}$.

Equations (1.1)-(1.3) all have the form

$$
Y=\sum_{F \in \mathcal{F}} X_{F} \psi_{F}+\varepsilon
$$

where $Y$ and $\varepsilon$ are random vectors of length $e, \mathcal{F}$ is a set of partitions of $\Omega$, and, for $F$ in $\mathcal{F}, \psi_{F}$ is a real vector of length $n_{F}$. Thus the expectation $\mathbb{E}(Y)$ of $Y$ is in the subspace $\sum_{F \in \mathcal{F}} V_{F}$.

There are two trivial partitions on $\Omega$, which are different when $e>1$. The parts of the equality partition $E$ are singletons, so $k_{E}=1, n_{E}=e$ and $X_{E}=I_{e}=P_{E}$. At the other extreme, the universal partition $U$ has a single part, so $n_{U}=1, k_{U}=e, X_{U} X_{U}^{\top}=J_{e e}$ and $P_{U}=e^{-1} J_{e e}$, where $J_{n m}$ denotes the $n \times m$ matrix with all entries equal to 1 . Moreover, $V_{E}$ is the whole space $\mathbf{R}^{\Omega}$, while $V_{U}$ is the 1-dimensional subspace of constant vectors. 
If $F$ and $G$ are two partitions of $\Omega$, their $n_{F} \times n_{G}$ incidence matrix $N_{F G}$ is defined by $N_{F G}=X_{F}^{\top} X_{G}$. The $(i, j)$-entry is the size of the intersection of the $i$-th part of $F$ with the $j$-th part of $G$. In particular, $N_{E F}=X_{F}$.

Given a set $\mathcal{F}$ of partitions of $\Omega$, denote by $\mathcal{A}_{\mathcal{F}}$ the algebra of $e \times e$ real matrices generated by the projection matrices $P_{F}$ for $F$ in $\mathcal{F}$, and denote by $\mathcal{J}_{\mathcal{F}}$ the algebra generated by the relation matrices $X_{F} X_{F}^{\top}$ for $F$ in $\mathcal{F}$. These are the same if all partitions in $\mathcal{F}$ are uniform. James called $\mathcal{J}_{\mathcal{F}}$ the relationship algebra of $\mathcal{F}$ in [99], but it was shown in [100, 115] that $\mathcal{A}_{\mathcal{F}}$ is more useful for understanding the properties of $\mathcal{F}$ relevant to a designed experiment.

\section{Refinement}

Definition If $F$ and $G$ are partitions of $\Omega$, then $F$ is finer than $G$ (equivalently, $G$ is coarser than $F$ ) if every part of $F$ is contained in a single part of $G$ but at least one part of $G$ is not a part of $F$. This relation is denoted $F \prec G$ or $G \succ F$.

In Example 1.3, $B \prec D$. If $F \prec G$ then $n_{F}>n_{G}$ and $V_{G}<V_{F}$.

Write $F \preccurlyeq G$ (or $G \succcurlyeq F$ ) to mean that either $F \prec G$ or $F=G$. Then $\preccurlyeq$ is a partial order. For every partition $F$, it is true that $E \preccurlyeq F \preccurlyeq U$ and $V_{U} \leq V_{F} \leq V_{E}$.

Proposition 3.1 Let $F$ and $G$ be partitions of $\Omega$. If $F \preccurlyeq G$ then $P_{F} P_{G}=$ $P_{G} P_{F}=P_{G}$.

As with any partial order, there is a choice about which of the two objects should be considered 'smaller'. Some statisticians write the refinement partial order in the opposite way to that used here. For example, see [31, 206, 207].

Since there are only a finite number of partitions of $\Omega$, there is no difficulty with the next definition.

Definition Let $F$ and $G$ be partitions of $\Omega$. The infimum $F \wedge G$ of $F$ and $G$ is the coarsest partition $H$ satisfying $H \preccurlyeq F$ and $H \preccurlyeq G$; its parts are the non-empty intersections of a part of $F$ and a part of $G$. Thus $F \wedge G=E$ if and only if no part of $F$ intersects any part of $G$ in more than one unit. The supremum $F \vee G$ of $F$ and $G$ is the finest partition $K$ satisfying $F \preccurlyeq K$ and $G \preccurlyeq K$; its parts are the connected components of the graph with vertex-set $\Omega$ and an edge between $\omega_{1}$ and $\omega_{2}$ if $F\left(\omega_{1}\right)=F\left(\omega_{2}\right)$ or $G\left(\omega_{1}\right)=G\left(\omega_{2}\right)$. 
Thus if $F \preccurlyeq G$ then $F \wedge G=F$ and $F \vee G=G$. In the design in Figure $4, R \wedge C=R \wedge L=C \wedge L=E$ and $R \vee C=R \vee L=C \vee L=U$.

Proposition 3.2 If $F$ and $G$ are partitions of $\Omega$ then $V_{F} \cap V_{G}=V_{F \vee G}$.

\section{Orthogonality}

\subsection{Definitions}

As Preece noted in [154], the word orthogonal has many different meanings in the statistical literature. Here I use the terminology in $[23,25,32$, 206].

Proposition 3.2 shows that subspaces $V_{F}$ and $V_{G}$ can never be orthogonal to each other. This motivates the following definition, from [206].

Definition Let $V$ and $W$ be subspaces of $\mathbf{R}^{\Omega}$. Then $V$ and $W$ are geometrically orthogonal to each other if the subspaces $V \cap(V \cap W)^{\perp}$ and $W \cap(V \cap W)^{\perp}$ are orthogonal to each other.

Proposition 4.1 Let $F$ and $G$ be partitions of $\Omega$. The following statements are equivalent:

(i) $V_{F}$ is geometrically orthogonal to $V_{G}$;

(ii) $P_{F} P_{G}=P_{G} P_{F}$;

(iii) $P_{F} P_{G}=P_{F \vee G}$;

(iv) for every unit $\omega$, we have $|F(\omega)||G(\omega)|=|(F \wedge G)(\omega)||(F \vee G)(\omega)|$.

The second statement above is sometimes called 'projectors commute', and the fourth 'proportional meeting within each class of the supremum'.

Definition Let $F$ and $G$ be partitions of $\Omega$. Then $F$ is orthogonal to $G$, written $F \perp G$, if $P_{F} P_{G}=P_{G} P_{F}$; and $F$ is strictly orthogonal to $G$, written $F \perp G$, if $P_{F} P_{G}=P_{G} P_{F}=P_{U}$.

Duquenne calls these two concepts local orthogonality and orthogonality respectively in [66]; the latter agrees with Gilliland's definition of orthogonality in [83]. Some authors split the definitions further according to whether or not $F \wedge G$ is uniform.

Proposition 3.1 shows that if $F \preccurlyeq G$ then $F \perp G$. In particular, all partitions are orthogonal to both $E$ and $U$, and every partition is orthogonal to itself. 


\begin{tabular}{|c|c|c|}
\hline$A \quad B$ & $\begin{array}{ll}B & C\end{array}$ & $A \quad C$ \\
\hline$C$ & $A$ & $B$ \\
\hline
\end{tabular}

Figure 5: A $2 \times 3$ row-column design with nine units and three letters, giving mutually orthogonal partitions into rows, columns and letters
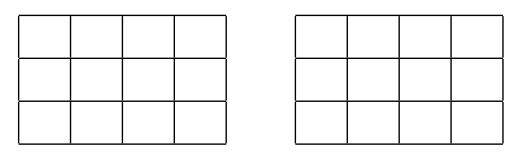

Figure 6: Two blocks, each of which is a $3 \times 4$ rectangle, so that there are 6 rows and 8 columns

In the design in Figure $4, R \perp C$. If $R, C$ and $L$ denote the partitions into rows, columns and letters in Figure 5, then $R \perp C, R \perp L$ and $C \perp L$ even though $R, R \wedge C$ and $R \wedge L$ are not uniform, because the 'proportional meeting' condition in Proposition 4.1(iv) is satisfied for all pairs and all pairwise suprema are equal to $U$. If $B, R$ and $C$ denote the partitions into blocks, rows and columns in Figure 6 , then $R \perp C$ but $R$ is not strictly orthogonal to $C$ because $R \vee C=B \neq U$.

Proposition 4.2 Let $F$ and $G$ be partitions of $\Omega$. Then $F \perp G$ if and only if $N_{F G}=e^{-1}\left(X_{F}^{\top} X_{F}\right) J_{n_{F} n_{G}}\left(X_{G}^{\top} X_{G}\right)$.

\subsection{Orthogonal arrays}

Definition An orthogonal array of strength two on $\Omega$ is a collection $\mathcal{F}$ of at least two uniform partitions of $\Omega$ with the property that every pair of distinct partitions is strictly orthogonal. Inductively, for $m \geq 3$, a collection $\mathcal{F}$ of at least $m$ partitions of $\Omega$ is an orthogonal array of strength $m$ if it is an orthogonal array of strength $m-1$ and, whenever $F_{1}, \ldots, F_{m}$ are distinct partitions in $\mathcal{F}$, the infimum $F_{1} \wedge F_{2} \wedge \cdots \wedge F_{m-1}$ is strictly orthogonal to $F_{m}$.

Figure 7 shows an orthogonal array of strength two with $e=12,|\mathcal{F}|=$ 11 , and $n_{F}=2$ for all $F$ in $\mathcal{F}$. It is equivalent to that given by Plackett and Burman [142]. Replacing each 0 by -1 and adjoining a row of $1 \mathrm{~s}$ gives a Hadamard matrix of order 12. The paper [142] inspired Rao to define orthogonal arrays and begin to develop a general theory of them in $[179,180]$. 


$\begin{array}{lllllllllllll}F_{1} & 0 & 0 & 1 & 0 & 0 & 0 & 1 & 1 & 1 & 0 & 1 & 1 \\ F_{2} & 1 & 0 & 0 & 1 & 0 & 0 & 0 & 1 & 1 & 1 & 0 & 1 \\ F_{3} & 0 & 1 & 0 & 0 & 1 & 0 & 0 & 0 & 1 & 1 & 1 & 1 \\ F_{4} & 1 & 0 & 1 & 0 & 0 & 1 & 0 & 0 & 0 & 1 & 1 & 1 \\ F_{5} & 1 & 1 & 0 & 1 & 0 & 0 & 1 & 0 & 0 & 0 & 1 & 1 \\ F_{6} & 1 & 1 & 1 & 0 & 1 & 0 & 0 & 1 & 0 & 0 & 0 & 1 \\ F_{7} & 0 & 1 & 1 & 1 & 0 & 1 & 0 & 0 & 1 & 0 & 0 & 1 \\ F_{8} & 0 & 0 & 1 & 1 & 1 & 0 & 1 & 0 & 0 & 1 & 0 & 1 \\ F_{9} & 0 & 0 & 0 & 1 & 1 & 1 & 0 & 1 & 0 & 0 & 1 & 1 \\ F_{10} & 1 & 0 & 0 & 0 & 1 & 1 & 1 & 0 & 1 & 0 & 0 & 1 \\ F_{11} & 0 & 1 & 0 & 0 & 0 & 1 & 1 & 1 & 0 & 1 & 0 & 1\end{array}$

Figure 7: Orthogonal array of strength two, consisting of 11 partitions of a set of size 12 into two parts: columns represent elements of the set, and each row shows one partition

For $n \geq 2$, the rows, columns and letters of any Latin square of order $n$ give an orthogonal array of strength two on a set of size $n^{2}$, with three partitions into parts of size $n$. See [95] for many uses and constructions of orthogonal arrays, as well as more theory. Eendebak and Schoen maintain a catalogue on the web page [76].

From Finney [77] onwards, finite Abelian groups have been a fruitful source of orthogonal arrays, under the name fractional factorial designs. For $i=1, \ldots, s$ let $G_{i}$ be an Abelian group of order $n_{i}$, where $n_{i} \geq 2$. Let $G$ be the product group $G_{1} \times G_{2} \times \cdots \times G_{s}$. Every complex irreducible character $\chi$ of $G$ has the form $\chi=\left(\chi_{1}, \chi_{2}, \ldots, \chi_{s}\right)$ where $\chi_{i}$ is an irreducible character of $G_{i}$ and $\chi\left(g_{1}, g_{2}, \ldots, g_{s}\right)=\chi_{1}\left(g_{1}\right) \chi_{2}\left(g_{2}\right) \cdots \chi_{s}\left(g_{s}\right)$. Let $H$ be a subgroup of $G$, and let $F_{i}$ be the partition of $H$ defined by the values of the $i$-th coordinate. Then $\left\{F_{1}, \ldots, F_{s}\right\}$ forms an orthogonal array of strength $m$ on $H$ if and only if the only non-trivial characters $\chi$ on $G$ whose restriction to $H$ is trivial have non-trivial components $\chi_{i}$ for at least $m+1$ values of $i$. For example, if $s=3, n_{1}=n_{2}=n_{3}=7$ and $G_{i}$ is $\mathbf{Z}_{7}$ written additively for $i=1,2$ and 3 then $\left\{F_{1}, F_{2}, F_{3}\right\}$ forms an orthogonal array of strength two on the subgroup $H=\left\{\left(g_{1}, g_{2}, g_{3}\right): g_{1}+g_{2}+g_{3}=0\right\}$. Up to isotopism (permutations of the names of the parts of each partition), this is the Latin square obtained as the Cayley table of $\mathbf{Z}_{7}$.

Some papers, such as $[61,112,141,213]$, call an orthogonal array regular if and only if it is made from an Abelian group in this way. There are two problems with this. The first is that, in each experiment, the parts of $F_{i}$ (such as varieties of lettuce) are unlikely to be labelled by the elements of a finite Abelian group. How is the statistician analysing the 


\begin{tabular}{|c|c|c|c|c|}
\hline$A$ & $B$ & $C$ & $D$ & $E$ \\
\hline$E$ & $A$ & $B$ & $C$ & $D$ \\
\hline$D$ & $E$ & $A$ & $B$ & $C$ \\
\hline$C$ & $D$ & $E$ & $A$ & $B$ \\
\hline$B$ & $C$ & $D$ & $E$ & $A$ \\
\hline
\end{tabular}

(a)

\begin{tabular}{|c|c|c|c|c|}
\hline$A$ & $B$ & $C$ & $D$ & $E$ \\
\hline$B$ & $A$ & $D$ & $E$ & $C$ \\
\hline$E$ & $D$ & $A$ & $C$ & $B$ \\
\hline$C$ & $E$ & $B$ & $A$ & $D$ \\
\hline$D$ & $C$ & $E$ & $B$ & $A$ \\
\hline
\end{tabular}

(b)

Figure 8: Two Latin squares of order five: square (a) is isotopic to the Cayley table of $\mathbf{Z}_{5}$, but square (b) is not

data to know whether or not the orthogonal array was constructed from Abelian groups? In any case, this derivation makes no difference to the data analysis. The second problem is that, for an experiment designed using a Latin square, it is usually of no practical importance whether or not the square is isotopic to the Cayley table of an Abelian group. As $n$ increases, so does the proportion of Latin squares of order $n$ which are not isotopic to such Cayley tables. Figure 8 shows two Latin squares of order five: only one of them is isotopic to a Cayley table.

The definitions in this section show that, in an orthogonal array $\mathcal{F}$ of strength two, $P_{F}$ commutes with $P_{G}$ for all $F$ and $G$ in $\mathcal{F}$. Proposition 4.1 shows that commutativity cannot be destroyed by inclusion of suprema. Thus Grömping and Bailey, in their paper [86] giving some more lenient definitions of regularity, proposed calling an orthogonal array geometrically regular if $P_{G_{1} \wedge \cdots \wedge G_{r}}$ commutes with $P_{H_{1} \wedge \cdots \wedge H_{s}}$ for all subsets $\left\{G_{1}, \ldots, G_{r}\right\}$ and $\left\{H_{1}, \ldots, H_{s}\right\}$ of $\mathcal{F}$. Thus the two orthogonal arrays in Figure 8 are geometrically regular, while the one in Figure 7 is not, because $F_{1} \wedge F_{2}$ is not orthogonal to $F_{3}$.

\subsection{Tjur block structures and orthogonal block structures}

Proposition 4.1 leads to these definitions, given in [23], building on the work in [206].

Definition Let $\mathcal{F}$ be a set of partitions on a finite set $\Omega$. Then $\mathcal{F}$ is a Tjur block structure if $\mathcal{F}$ is closed under taking suprema, every pair of partitions in $\mathcal{F}$ is orthogonal, and $E \in \mathcal{F}$. If, in addition, $\mathcal{F}$ is closed under taking infima, every partition in $\mathcal{F}$ is uniform, and $U \in \mathcal{F}$, then $\mathcal{F}$ is an orthogonal block structure.

For example, if $\mathcal{F}$ is an orthogonal array of strength two then $\mathcal{F} \cup\{E, U\}$ is a Tjur block structure. 
For $F$ in $\mathcal{F}$, define a further subspace $W_{F}$ of $\mathbf{R}^{\Omega}$ by

$$
W_{F}=V_{F} \cap \bigcap_{F \prec G \in \mathcal{F}} V_{G}^{\perp}=V_{F} \cap\left(\sum_{F \prec G \in \mathcal{F}} V_{G}\right)^{\perp} .
$$

Theorem 4.3 If $\mathcal{F}$ is a Tjur block structure then the subspaces $W_{F}$, for $F$ in $\mathcal{F}$, are mutually orthogonal and their sum is $\mathbf{R}^{\Omega}$.

It follows that the dimensions of the subspaces $W_{F}$, and the matrices of orthogonal projection onto them, can be calculated recursively, starting with the coarsest partition in $\mathcal{F}$. Moreover, the algebra $\mathcal{A}_{\mathcal{F}}$ is commutative, and consists of all real linear combinations of the matrices $P_{F}$, for $F$ in $\mathcal{F}$. The subspaces $W_{F}$ are the mutual eigenspaces of $\mathcal{A}_{\mathcal{F}}$. For any partition in $\mathcal{F}$ which is uniform, its relation matrix is also in $\mathcal{A}_{\mathcal{F}}$.

Tjur block structures are used widely in statistics, in two different contexts, which are explained more in Section 6. One concerns covariance, and the other expectation.

The covariance $\operatorname{cov}\left(Y_{\alpha}, Y_{\beta}\right)$ of responses $Y_{\alpha}$ and $Y_{\beta}$ is defined to be $\mathbb{E}\left[\left(Y_{\alpha}-\mathbb{E}\left(Y_{\alpha}\right)\right)\left(Y_{\beta}-\mathbb{E}\left(Y_{\beta}\right)\right)\right]$. The variance-covariance matrix $\operatorname{Cov}(Y)$ of the random vector $Y$ in equation (2.1) is the $e \times e$ matrix whose entry in row $\alpha$ and column $\beta$ is $\operatorname{cov}\left(Y_{\alpha}, Y_{\beta}\right)$. It is often assumed that $\operatorname{Cov}(Y)$ is an unknown matrix in $\mathcal{J}_{\mathcal{H}}$ for a specified Tjur block structure $\mathcal{H}$ with $\mathcal{H} \subseteq \mathcal{F}$. If the partitions are all uniform then $\mathcal{J}_{\mathcal{H}}=\mathcal{A}_{\mathcal{H}}$ and so the eigenspaces of $\operatorname{Cov}(Y)$ are known. Then closure under suprema ensures that there is no pre-determined linear dependence among the eigenvalues, which avoids complications in estimating their values: see [35].

The other use is to give a collection of models for the expectation $\mathbb{E}(Y)$ of $Y$. It is assumed, as in equations (1.1)-(1.3), that there is a subset $\mathcal{G}$ of $\mathcal{F}$ such that $\mathbb{E}(Y) \in \sum_{G \in \mathcal{G}} V_{G}$, and we would like to find the smallest such $\mathcal{G}$ : see [28]. Closure under suprema is essential for the existence of such a smallest subset.

The set of partitions $\{E, R, C, L, U\}$ in any Latin square forms an orthogonal block structure. Apart from Latin squares, and sets of mutually orthogonal Latin squares, most orthogonal block structures in common use are poset block structures, described in the next subsection.

\subsection{Poset block structures}

Apart from Großmann's AutomaticAnova [87], and the package recently introduced by Bate and Chatfield [42, 43], most statistical software cannot currently recognise the $\preccurlyeq$ relation, unless the names of the partitions contain a clue. For example, if $R, C$ and $L$ denote the partitions of 
a Latin square into rows, columns and letters respectively, then standard analysis of variance in the popular statistical software $\mathrm{R}$ [175] can recognise that $R \wedge C \preccurlyeq R$ and $R \wedge C \preccurlyeq C$ but not that $R \wedge C \preccurlyeq L$; nor can it recognise that $H \preccurlyeq R$ if $H$ is another name for the partition $R \wedge C$. Each software has its own symbol for the binary operator " $\wedge$ ", usually something like "." or ":" that is available on standard keyboards. The usual rule seems to be that $A_{1} \wedge A_{2} \wedge \cdots \wedge A_{n}$ is recognised to be finer than $B_{1} \wedge B_{2} \wedge \cdots \wedge B_{m}$ if and only if $\left\{B_{1}, B_{2}, \ldots, B_{m}\right\}$ is a proper subset of $\left\{A_{1}, A_{2}, \ldots, A_{n}\right\}$. This rule is true for so-called poset block structures when their partitions are named canonically.

The definition of poset block structures needs another partial order, which I shall write as $\sqsubseteq$. If $(\mathcal{P}, \sqsubseteq)$ is a partially ordered set (poset for short), a subset $\mathcal{Q}$ of $\mathcal{P}$ is defined to be ancestral, or an up-set, if whenever $i \in \mathcal{Q}$ and $j \in \mathcal{P}$ with $i \sqsubset j$ then $j \in \mathcal{Q}$.

Definition Let $\mathcal{P}=\{1, \ldots, s\}$ be a finite set with a partial order $\sqsubseteq$. For $i=1, \ldots, s$, let $\Omega_{i}$ be a finite set of size $n_{i}$, where $n_{i} \geq 2$. Put $\Omega=\Omega_{1} \times \Omega_{2} \times \cdots \times \Omega_{s}$. Let $F_{i}$ be the partition of $\Omega$ defined by the values of the $i$-th coordinate, for $i=1, \ldots, s$. If $\mathcal{Q} \subseteq \mathcal{P}$, define the partition $F_{\mathcal{Q}}$ of $\Omega$ by $F_{\mathcal{Q}}=\bigwedge_{i \in \mathcal{Q}} F_{i}$. The poset block structure on $\Omega$ defined by $(\mathcal{P}, \sqsubseteq)$ is $\left\{F_{\mathcal{Q}}: \mathcal{Q}\right.$ is an ancestral subset of $\left.\mathcal{P}\right\}$.

Example 4.4 If $\mathcal{P}=\{1,2,3\}$ with $1 \sqsupset 2$ and $1 \sqsupset 3$ then the corresponding poset block structure may be visualized as $n_{1}$ rectangles (parts of $F_{\{1\}}$ ) each defined by $n_{2}$ rows (parts of $F_{\{1,2\}}$ ) and $n_{3}$ columns (parts of $F_{\{1,3\}}$ ). Figure 6 shows an example with $n_{1}=2, n_{2}=3$ and $n_{3}=4$.

Example 4.5 Extend Example 1.4 so that there are 14 people, of whom seven are men and seven are women. If we ignore the partition into exercise regimes, we have the poset block structure defined by $\{1,2,3\}$ with $n_{1}=2$, $n_{2}=7, n_{3}=4$ and $2 \sqsubset 1$. The parts of $F_{\{1\}}$ are the genders; the parts of $F_{\{1,2\}}$ are the people; the parts of $F_{\{3\}}$ are the months; each part of $F_{\{1,3\}}$ is one gender for one month; and the parts of $F_{\{1,2,3\}}$ are the units.

Theorem 4.6 The following statements hold for any poset block structure defined by a poset $(\mathcal{P}, \sqsubseteq)$.

(i) If $\mathcal{Q}$ is an ancestral subset of $\mathcal{P}$, then $F_{\mathcal{Q}}$ is uniform, with all parts of size $\prod_{i \in \mathcal{P} \backslash \mathcal{Q}} n_{i}$.

(ii) The subsets $\emptyset$ and $\mathcal{P}$ are both ancestral. Moreover, $F_{\emptyset}=U$ and $F_{\mathcal{P}}=E$. 
(iii) If $\mathcal{Q}$ and $\mathcal{R}$ are both ancestral subsets of $\mathcal{P}$, then $F_{\mathcal{Q}} \perp F_{\mathcal{R}}, F_{\mathcal{Q}} \vee F_{\mathcal{R}}=$ $F_{\mathcal{Q} \cap \mathcal{R}}$ and $F_{\mathcal{Q}} \wedge F_{\mathcal{R}}=F_{\mathcal{Q} \cup \mathcal{R}}$

It follows that every poset block structure is an orthogonal block structure. Latin squares show that the converse is not true.

Poset block structures were investigated extensively (but not so named) by Yates [224] and later by Kempthorne and his colleagues [107, 205, 232], but these authors did not manage to completely distinguish between the two partial orders involved. By restricting himself to series-parallel posets, Nelder was able to provide recursive definitions and constructions in [126] for what he called simple orthogonal block structures. This approach led to algorithms that underlie many different programs used today for the analysis of variance. Speed and Bailey pointed out in [197, 198] that Nelder's approach can be used for arbitrary finite posets. Further details and examples are in $[23,26,30]$.

\section{Balance}

In this section we denote by $B$ and $L$ two partitions of $\Omega$ whose parts will be called blocks and letters respectively.

Definition The relationship between $L$ and $B$ is binary if $L \wedge B=E$; this means that each letter occurs at most once in each block. It is generalized binary if no two intersections of a part of $L$ with a part of $B$ differ in size by more than one.

Confusion alert! The first of these really is a binary relation whose name is 'binary'.

An $n \times n$ matrix is called completely symmetric if it is a linear combination of $I_{n}$ and $J_{n n}$.

\subsection{Combinatorial notions of balance}

Let $i$ and $j$ be two letters, not necessarily distinct. The number of ordered pairs $\left(\omega_{1}, \omega_{2}\right)$ in $\Omega \times \Omega$ with the properties that $B\left(\omega_{1}\right)=B\left(\omega_{2}\right)$, $L\left(\omega_{1}\right)=i$ and $L\left(\omega_{2}\right)=j$ is equal to the $(i, j)$-entry of $N_{L B} N_{B L}$. It is called the concurrence of $i$ and $j$ in blocks.

The classical definition of balance, given by Yates in [225], follows.

Definition If the partition $B$ is uniform and $L \wedge B=E$ then the partition $L$ is balanced with respect to $B$ if the off-diagonal elements of $N_{L B} N_{B L}$ are all the same but not zero. 
For a binary design, a counting argument shows that if $L$ is balanced with respect to $B$ then $L$ is also uniform and hence that $N_{L B} N_{B L}$ is completely symmetric. This definition of balance includes as a special case complete-block designs. In these, $L$ is uniform, $n_{B} n_{L}=e, N_{L B} N_{B L}=$ $n_{B} J_{n_{L} n_{L}}$ and $L \perp B$. For all other designs which are balanced according to this definition, the coefficient of $I_{n_{L}}$ in $N_{L B} N_{B L}$ is non-zero and therefore $N_{L B} N_{B L}$ has rank $n_{L}$.

Fisher proved his famous inequality in [79]: if $L \wedge B=E, B$ is uniform, and $L$ is balanced with respect to $B$ but not orthogonal to $B$, then $n_{L} \leq$ $n_{B}$. There are now many proofs of this result. One of the simplest is the observation that the rank of $N_{L B} N_{B L}$ cannot be greater than the number of columns of $N_{L B}$. Conversely, if $N_{L B} N_{B L}$ has rank $n_{L}$ and $n_{L}=n_{B}$, it follows that $N_{L B}$ is invertible: hence if $N_{L B} N_{B L}$ is completely symmetric then so is $N_{B L} N_{L B}$ and therefore $B$ is also balanced with respect to $L$. See [52, Chapter 1] and [202, Chapter 2].

How should this definition be generalized if $B$ is not uniform or the relationship between $L$ and $B$ is not binary? Relaxing the uniformity of $B$ gives pairwise balanced designs, introduced in [110]. Now a counting argument shows that the entries on the diagonal of $N_{L B} N_{B L}$ are all strictly bigger than the common off-diagonal entry, and so $N_{L B} N_{B L}$ is positive definite; therefore it has rank $n_{L}$, and Fisher's inequality follows as before. As [223] shows, pairwise balanced designs have been a very fruitful field of research, which includes results about the existence of BIBDs. However, as we show in the next subsection, this notion of balance does not match what is needed from the statistical point of view.

\subsection{Statistical notions of balance}

The vector form of equation (1.1) is $Y=X_{B} \beta+X_{L} \tau+\varepsilon$. To estimate the vector $\tau$ up to an additive constant, it is necessary to project the data vector onto the subspace $\left(V_{L}+V_{B}\right) \cap V_{B}^{\perp}$. The $n_{L} \times n_{L}$ information matrix $C_{L B}$ is defined by $C_{L B}=X_{L}^{\top}\left(I_{e}-P_{B}\right) X_{L}$. Note that $I_{e}-P_{B}$ is the matrix of orthogonal projection onto $V_{B}^{\perp}$. Also, if $B$ is uniform then $X_{L}^{\top} P_{B} X_{L}=k_{B}^{-1} N_{L B} N_{B L}$.

The matrix $C_{L B}$ is symmetric, with row-sums zero, so it is singular. If $B \preccurlyeq L$ then it is impossible to estimate any difference $\tau_{i}-\tau_{j}$. In this case, $C_{L B}=0$, and the block design is not considered to be balanced. If $C_{L B}$ has rank $n_{L}-1$ then all differences $\tau_{i}-\tau_{j}$ can be estimated and $C_{L B}$ has a Moore-Penrose generalized inverse $C_{L B}^{-}$. Under the assumption that $\operatorname{Cov}(Y)=\sigma^{2} I_{e}$, standard linear model theory shows that the variance of the estimator of $\tau_{i}-\tau_{j}$ is

$$
\left(C_{L B}^{-}(i, i)+C_{L B}^{-}(j, j)-C_{L B}^{-}(i, j)-C_{L B}^{-}(j, i)\right) \sigma^{2} .
$$




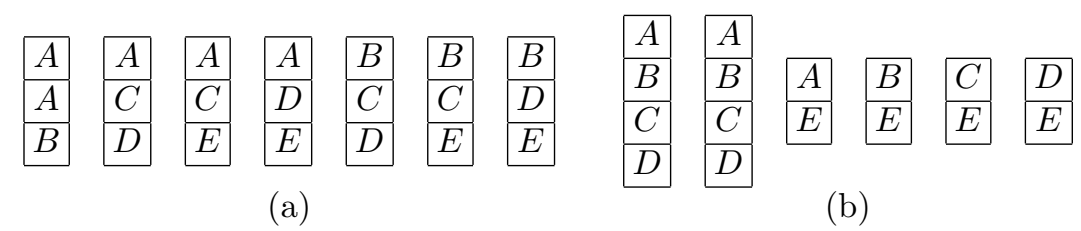

Figure 9: Two variance-balanced block designs: columns represent blocks

See [33] for further explanation for cominatorialists. Thus a block design is called variance-balanced if $C_{L B}$ is completely symmetric but not zero: this terminology was introduced by Tocher in [208]. In this case both $C_{L B}$ and $C_{L B}^{-}$are scalar multiples of $n_{L} I_{n_{L}}-J_{n_{L} n_{L}}$.

Figure 9 shows two block designs which are variance-balanced but are not BIBDs. In the design in Figure 9(a), taken from [33, 208], $B$ is uniform but the relationship between $L$ and $B$ is not binary, even though $k_{B}=$ $3<5=n_{L}$. In the design in Figure $9(\mathrm{~b}), B$ is not uniform.

If a block design is variance-balanced then the off-diagonal entries of $X_{L}^{\top} P_{B} X_{L}$ are all equal. If $L$ is not orthogonal to $B$, a counting argument similar to that used for pairwise balanced designs shows that every diagonal entry is strictly bigger than this common value, and so $X_{L}^{\top} P_{B} X_{L}$ has rank $n_{L}$. Since $P_{B}$ has rank $n_{B}$, this shows that $n_{B} \geq n_{L}$, so that Fisher's inequality holds for variance-balanced designs. If, in a variance-balanced design, $B$ is also uniform and $n_{B}=n_{L}$, then the argument in Section 5.1 shows that the design obtained by interchanging the roles of $B$ and $L$ is also variance-balanced.

Hedayat and Federer [92] gave examples to show that neither of pairwise balance and variance balance implies the other.

If the experimenter is more interested in estimating some differences of the form $\tau_{i}-\tau_{j}$ than others, the experiment may well be designed so that $L$ is not uniform. If there are no blocks, then the information matrix is $C_{L U}$, defined by $C_{L U}=X_{L}^{\top}\left(I_{e}-P_{U}\right) X_{L}=X_{L}^{\top} X_{L}-e^{-1} X_{L}^{\top} J_{e e} X_{L}$. In a block design, the efficiency for the estimation of $\tau_{i}-\tau_{j}$ is the ratio of the variance in an unblocked design, in which each part of $L$ has the same size as it does in the block design, to that in the block design, assuming that the value of $\sigma^{2}$ is unchanged: see [135]. The block design is said to be efficiency-balanced if $C_{L B}$ is a scalar multiple of $C_{L U}$. This concept was introduced by Jones in [105], but not named until later. In the terminology of [100], the partition $L$ has first order balance with respect to $B$.

One easy construction of an efficiency-balanced block design is to take 


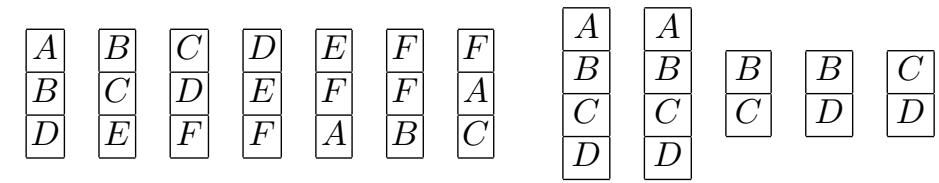

(a) (b)

Figure 10: Two efficiency-balanced designs: columns represent blocks

a BIBD and identify two letters. For example, this gives the non-binary design in Figure 10(a). Figure 10(b) shows an binary efficiency-balanced block design where $B$ is not uniform; it is taken from [219].

If $L$ is not orthogonal to $B$, the information matrices $C_{L B}$ and $C_{L U}$ cannot be equal. Hence, if one is a scalar multiple of the other then $X_{L}^{\top} X_{L}$ is a linear combination of $X_{L}^{\top} P_{B} X_{L}$ and $X_{L}^{\top} P_{U} X_{L}$. The diagonal matrix $X_{L}^{\top} X_{L}$ has rank $n_{L}$, while the rank of any linear combination of $X_{L}^{\top} P_{B} X_{L}$ and $X_{L}^{\top} P_{U} X_{L}$ is bounded above by $n_{B}$, so, once again, Fisher's inequality holds.

\subsection{Balance between partitions}

As Figures 9 and 10 show, block designs which are variance-balanced or efficiency-balanced tend to have either one or both of the partitions $L$ and $B$ being non-uniform. In fact, if $L$ is uniform then variance balance is equivalent to efficiency balance; otherwise, it is impossible for a design to have both properties. On the other hand, adjoining two BIBDs with different block sizes gives a design which is pairwise balanced, variancebalanced and efficiency-balanced but has non-uniform partition $B$.

These considerations motivate the following definition.

Definition Let $L$ and $B$ be uniform partitions of $\Omega$. Then $L$ is balanced with respect to $B$ if $X_{L}^{\top}\left(I_{e}-P_{B}\right) X_{L}$ is completely symmetric but not zero. It is strictly balanced if it is balanced and the relationship between $L$ and $B$ is generalized binary.

The second part of this definition follows [25]. Strict balance is called balance by Kiefer, who showed in [108] that strictly balanced block designs are optimal in the sense, described more fully in $[33,191]$, that the average variance of the estimators of differences like $\tau_{i}-\tau_{j}$ is minimized. Binary balance is called total balance in [98, 134, 145, 147]. If $L \perp B$ and $L \wedge B$ is uniform then $L$ is balanced with respect to $B$ and $B$ is balanced with respect to $L$. 


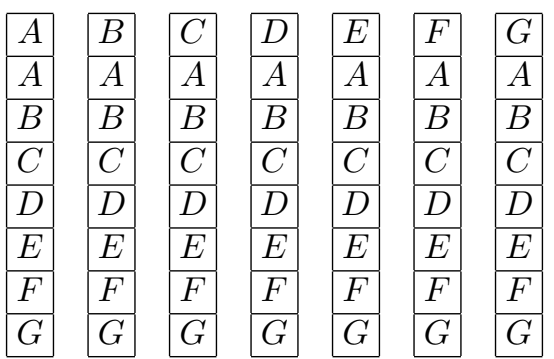

(a)

\begin{tabular}{|c|c|c|c|c|c|c|}
\hline$A$ & $B$ & $C$ & $D$ & $E$ & $F$ & $G$ \\
\hline$A$ & $B$ & $C$ & $D$ & $E$ & $F$ & $G$ \\
\hline $\bar{C}$ & $D$ & $E$ & $F$ & $G$ & $A$ & $B$ \\
\hline $\bar{C}$ & $D$ & $E$ & $F$ & $G$ & $A$ & $B$ \\
\hline$D$ & $E$ & $F$ & $G$ & $\bar{A}$ & $B$ & $\bar{C}$ \\
\hline$D$ & $E$ & $F$ & $G$ & $A$ & $B$ & $C$ \\
\hline$E$ & $F$ & $G$ & $A$ & $B$ & $C$ & $D$ \\
\hline$E$ & $F$ & $G$ & $A$ & $B$ & $C$ & $D$ \\
\hline
\end{tabular}

(b)

Figure 11: Two block designs in which letters are balanced with respect to blocks, which are represented by columns

In general, balance is not a symmetric relation, unlike orthogonality. To emphasize this, here we write $L \backslash B$ or $B \triangleleft L$ to indicate that $L$ is balanced with respect to $B$ but not strictly orthogonal to $B$, with $\boldsymbol{\nabla}$ and $\varangle$ replaced by $\triangleright$ and $\triangleleft$ for strict balance. If $L$ and $B$ are both strictly balanced with respect to each other but not orthogonal to each other, we write $L \bowtie B$.

Example 5.1 Suppose that $e=56$ and $n_{B}=n_{L}=7$. Figure 11 shows two block designs in which $L \gg B$. Neither is binary. The one in Figure 11(a) has strict balance, but the one in Figure 11(b) does not.

The results in Section 5.2 show that if $L \gg B$ then $n_{L} \leq n_{B}$, and that if, in addition, $n_{L}=n_{B}$, then $B \triangleright L$.

\section{Linear Models}

\subsection{Fixed effects and random effects}

As explained briefly in equation (2.1), in an experiment whose design is defined by a set $\mathcal{F}$ of partitions, it is usually assumed that the response data, which form a vector in $\mathbf{R}^{e}$, give a realization of a random vector $Y$ which satisfies

$$
Y=\sum_{F \in \mathcal{F}} X_{F} \psi_{F}+\varepsilon
$$

Here $\varepsilon$, which can be regarded as $\psi_{E}$, is a random vector of length $e$ with zero mean, and $\operatorname{Cov}(\varepsilon)=\sigma^{2} I_{e}$. For some partitions $F$ we assume that 
$\psi_{F}$ is a real vector of length $n_{F}$. Then $F$ is said to have fixed effects. The other possibility is that $\psi_{F}$ is a random vector with zero mean and $\operatorname{Cov}\left(\psi_{F}\right)=\sigma_{F}^{2} I_{n_{F}}$. Then $F$ is said to have random effects.

Equation (6.1), together with the assumptions about fixed and random effects, are called the linear model for the data.

When all effects (apart from $E$ ) are fixed, the main step in estimating the vector $\psi_{F}$ (up to an additive constant) is the projection of the data vector onto the subspace

$$
\sum_{H \in \mathcal{F}} V_{H} \cap\left(\sum_{G \in \mathcal{F} \backslash\{F\}} V_{G}\right)^{\perp} .
$$

Section 7 gives more details about the subspaces involved. Sections 8 and 9 discuss combinatorial conditions necessary for the projections to have good properties.

\subsection{Partitions related by refinement}

In the discussion of equation (1.2) in Section 1, we saw that if $F \prec G$ then there is a potential difficulty in including both $X_{F} \psi_{F}$ and $X_{G} \psi_{G}$ in the linear model, because $V_{G}<V_{F}$. Here is an explanation of how this is handled in three common situations.

6.2.1 Nested block designs If $F$ and $G$ are both inherent and $F \prec G$ then we have a situation like that in Example 1.3. In the notation used there, $B \prec D$ and the parts of $B$ and $D$ can be thought of as small blocks and large blocks respectively. Some people say that the small blocks are nested in the large blocks. A third partition, $L$, is the one in which the experimenter is actually interested. Put $\tau=\psi_{L}$.

It is common to assume that $L$ and $D$ have fixed effects while $B$ has random effects, because if $B$ has fixed effects then the relation between $L$ and $D$ is immaterial. Then one simple estimate of $\tau$, up to an additive constant, can be obtained by projecting the data vector onto $V_{B}^{\perp}$. If $L$ is not orthogonal to $B$, another can be obtained by projecting the data onto $V_{B} \cap V_{D}^{\perp}$. The variances of these estimators are proportional to $\sigma^{2}$ and $\sigma^{2}+k_{B} \sigma_{B}^{2}$ respectively. Once the quantities $\sigma^{2}$ and $\sigma_{B}^{2}$ have been estimated from the data, an appropriate linear combination of these two estimates of $\tau$ gives a better estimate: see [24, 128].

In a variant of this, $D$ is also assumed to have random effects. If $L$ is not orthogonal to $D$, a third estimate of $\tau$ is obtained by projecting the data onto $V_{D}$, and then all three estimates are combined. 
6.2.2 Split-plot designs There are some circumstances where $F$ is inherent and $G$ is not, but practical constraints force $F \prec G$. In Example 1.6, the gardeners might object that it is too cumbersome for any of them to use more than one watering regime. If the parts of $B$ and $R$ are gardens and watering regimes respectively, then $B$ is inherent, $R$ is not, and $B \prec R$. Now we assume that $B$ has random effects and $R$ has fixed effects. The vector $\psi_{R}$ is estimated from the projection of the data onto $V_{B}$.

To understand the common name for these designs, rename the gardens as plots. There is only one watering regime on each plot but, in Example 1.6, each plot is split up into three patches, and different lettuce varieties are grown on each patch.

6.2.3 Simpler models If $F$ is allocated by the experimenter and $G$ is an innate grouping of the parts of $F$, then there is no avoiding the relation $F \prec G$ even when $F$ and $G$ both have fixed effects. For example, suppose that the parts of $F$ are the exercise regimes in Example 1.5. If five of these have individual exercise while five involve activity with other people, this gives a partition $G$ which groups the parts of $F$ into two groups of five.

Now the linear model which includes $X_{G} \psi_{G}$ but not $X_{F} \psi_{F}$ is a submodel of the one that includes $X_{F} \psi_{F}$ but not $X_{G} \psi_{G}$. Using the former gives an estimate of $\psi_{G}$ from the data, while using the latter gives an estimate of $\psi_{F}$. Because $V_{G}<V_{F}$, the projection of the data vector onto $V_{F}$ is no further from the original data than the projection onto $V_{G}$, and so it is clear that the model which includes $X_{F} \psi_{F}$ provides a better fit to the data. However, the improvement might be no more than could easily happen by chance. The subtle statistical business of hypothesis testing addresses the question "Can we attribute the different effects of different exercise regimes to the simple distinction between communal activity and solo activity?"

\section{Subspaces}

In this section we examine the subspaces derived from two or more subspaces of a real vector space with an inner product. The theory applies to subspaces of any sort, but we shall present it for subspaces defined by partitions as in Section 2.

\subsection{Two subspaces}

In [100], James and Wilkinson examined the further subspaces defined by $V_{F}$ and $V_{G}$ for any pair of partitions $F$ and $G$ of $\Omega$, building on the 
algebra in [99]. The subscript notation that follows is my own responsibility. Put $V_{F-G}=V_{F} \cap V_{G}^{\perp}$. Since $V_{F \vee G}=V_{F} \cap V_{G}$ by Proposition 3.2, the subspaces $V_{F-G}$ and $V_{F \vee G}$ are orthogonal to each other, and both are subspaces of $V_{F}$. Put $V_{F G}=V_{F} \cap\left(V_{F-G}\right)^{\perp} \cap V_{F \vee G}^{\perp}$. Denote by $V_{F \vdash G}$ the image of the projection of $V_{F}$ onto $V_{G}^{\perp}$, which is $\left(V_{F}+V_{G}\right) \cap V_{G}^{\perp}$. Define $V_{G-F}, V_{G F}$ and $V_{G \vdash F}$ analogously.

If $\mathbb{E}(Y)=X_{F} \psi_{F}+X_{G} \psi_{G}$ then, in order to estimate the vector $\psi_{F}$ (up to addition of a vector in $V_{F \vee G}$ ), it is necessary to project the data onto $V_{F \vdash G}$.

Put $Q_{F}=P_{F}-P_{F \vee G}$, which is the matrix of orthogonal projection onto $V_{F} \cap V_{F \vee G}^{\perp}$, and $Q_{G}=P_{G}-P_{F \vee G}$. The following results are in $[25,99,100]$.

Theorem 7.1 $\quad$ (i) $V_{F}$ is the orthgonal direct sum of $V_{F \vee G}, V_{F-G}$ and $V_{F G}$. Hence $n_{F}=\operatorname{dim}\left(V_{F}\right)=n_{F \vee G}+\operatorname{dim}\left(V_{F-G}\right)+\operatorname{dim}\left(V_{F G}\right)$.

(ii) The column space of $Q_{F} Q_{G}$ is $V_{F G}$ and the column space of $Q_{G} Q_{F}$ is $V_{G F}$. Hence $\operatorname{dim}\left(V_{F G}\right)=\operatorname{dim}\left(V_{G F}\right)$.

(iii) If $F \perp G$ then the subspaces $V_{F G}$ and $V_{G F}$ are both zero.

(iv) Let $x$ be an eigenvector of $Q_{F} Q_{G} Q_{F}$ with non-zero eigenvalue $\lambda$, and let $\theta$ be the angle between $x$ and $Q_{G} x$. Then $\cos ^{2} \theta=\lambda$. Moreover, the projection of $x$ onto $V_{F \vdash G}$ is $x-Q_{G} x$, the angle between this and $x$ is $\pi / 2-\theta$, and $x$ is an eigenvector of $Q_{F}\left(I_{e}-Q_{G}\right) Q_{F}$ with eigenvalue $1-\lambda$.

(v) If $F$ is balanced with respect to $G$ then $F \vee G=U$ and every vector in $V_{F} \cap V_{U}^{\perp}$ is an eigenvector of $Q_{F} Q_{G} Q_{F}$. Hence either $F \perp G$ and $V_{F-G}=V_{F} \cap V_{U}^{\perp}$ or $F$ is not orthogonal to $G$ and $V_{F-G}=\{0\}$. In the second case, the unique eigenvalue $\lambda$ is in $(0,1), Q_{F} Q_{G} Q_{F}=$ $\lambda Q_{F}$, and the matrix of orthogonal projection onto $\left(V_{F}+V_{G}\right) \cap V_{U}^{\perp}$ is

$$
Q_{G}+(1-\lambda)^{-1}\left(Q_{F}-Q_{G} Q_{F}-Q_{F} Q_{G}+Q_{G} Q_{F} Q_{G}\right),
$$

which simplifies to

$$
(1-\lambda)^{-1}\left(Q_{F}+Q_{G}-Q_{F} Q_{G}-Q_{G} Q_{F}\right)
$$

if $n_{F}=n_{G}$; moreover, if $F \wedge G=E$ then $\lambda=\left(n_{F}-k_{G}\right) /\left[\left(n_{F}-1\right) k_{G}\right]$.

(vi) The column space of $\left(I_{e}-Q_{G}\right) Q_{F}$ is $V_{F \vdash G}$.

(vii) $V_{F-G} \leq V_{F \vdash G}$, and the orthogonal complement of $V_{F-G}$ in $V_{F \vdash G}$ is $\left(V_{F G}+V_{G F}\right) \cap V_{G}^{\perp}$, which has dimension equal to $\operatorname{dim}\left(V_{F G}\right)$. 
Parts (i), (ii) and (v) give yet another proof of Fisher's inequality. If $F \triangleright G$ then $n_{F}-1=\operatorname{dim}\left(V_{F G}\right)=\operatorname{dim}\left(V_{G F}\right) \leq n_{G}-1$.

\subsection{Three subspaces but no refinement relation}

When there are three or more partitions, the statistical issues are more affected by which are inherent and which are of interest. This has been discussed in Section 6.2 for the case that one partition is finer than another. Here we assume that the partitions are $R, C$ and $L$, with no relation of refinement among them. For simplicity of exposition, assume that $R \vee C=$ $L \vee R=L \vee C=U$, so that $V_{R} \cap V_{C}=V_{L} \cap V_{R}=V_{L} \cap V_{C}=V_{U}$. Then the design in Figure 4 provides a working example.

Now Equation (6.1) becomes

$$
Y=X_{R} \alpha+X_{C} \phi+X_{L} \tau+\varepsilon .
$$

Put $Q_{R}=P_{R}-P_{U}, Q_{C}=P_{C}-P_{U}$ and $Q_{L}=P_{L}-P_{U}$, which are the matrices of orthogonal projection onto $V_{R} \cap V_{U}^{\perp}, V_{C} \cap V_{U}^{\perp}$ and $V_{L} \cap V_{U}^{\perp}$.

7.2.1 Row-column designs In the most common use of such a design in experiments, rows and columns are inherent, with $R \wedge C=E$ and $R \perp C$. The experimenter chooses the partition into letters and wants to estimate $\tau$ up to an additive constant.

If rows, columns and letters all have fixed effects and we want to estimate $\tau$, then we have to project the data onto $\left(V_{R}+V_{C}\right)^{\perp}$. Since $R \perp C$, the matrix of this projection is $I_{e}-Q_{R}-Q_{C}-P_{U}$. If $L>R$ and $L \triangleright C$ then $V_{L R}=V_{L C}=V_{L} \cap V_{U}^{\perp}$ and $Q_{L}\left(I_{e}-Q_{R}-Q_{C}-P_{U}\right) Q_{L}$ is a scalar multiple of $Q_{L}$. Unless the scalar is zero, this implies that this design has variance balance for the estimation of $\tau$.

On the other hand, if rows and columns both have random effects then further estimates of $\tau$ can be obtained by projecting the data onto $V_{R} \cap V_{U}^{\perp}$ and $V_{C} \cap V_{U}^{\perp}$. If $L \gg R$ and $L \triangleright C$ then, again, $Q_{L} Q_{R} Q_{L}$ and $Q_{L} Q_{C} Q_{L}$ are both non-zero scalar multiples of $Q_{L}$, and so the combined estimates of $\tau$ still have variance balance.

In this case, $Q_{L} Q_{R} Q_{L}$ commutes with $Q_{L} Q_{C} Q_{L}$. This property is called general balance by Nelder in [127]. There is not room here for a full discussion of general balance, but I will mention another special case that is very useful if it is not possible for $L$ to be orthogonal to or balanced with respect to both $R$ and $C$, for example if $n_{L}>n_{R}$ and $n_{L}>n_{C}$.

Suppose that $V_{L R} \perp V_{L C}$. If $x \in V_{L R}$ then $x \in V_{C}^{\perp}$ and so the linear combination $x^{\top} X_{L} \tau$ can be estimated in $\left(V_{R}+V_{C}\right)^{\perp}$ and in $V_{R} \cap V_{U}^{\perp}$ but not in $V_{C} \cap V_{U}^{\perp}$. There is an analogous conclusion if $x \in V_{C R}$. On the 
other hand, if $x \in V_{L} \cap\left(V_{U}+V_{L R}+V_{L C}\right)^{\perp}$ then $x^{\top} X_{L} \tau$ is estimated only in $\left(V_{R}+V_{C}\right)^{\perp}$. Thus each linear combination is estimated by combining at most two simple estimates, which is sometimes considered an advantage. This property is discussed further in Section 8.

7.2.2 Block designs for two non-interacting sets of treatments In the other main use of a design like the one in Figure 4, the partition into letters is inherent (and letters are called 'blocks'), the experimenter chooses the other two partitions and wants to estimate $\alpha$ and $\phi$ up to additive constants. It is desirable that $R \perp C$, so that every part of $R$ occurs with every part of $C$ equally often.

Usually it is assumed that $R, C$ and $L$ all have fixed effects. If we ignore $C$, a simplistic method of estimating $\alpha$ is to project the data onto $V_{R \vdash L}$. If this subspace is not orthogonal to $V_{C \vdash L}$ then this estimate of $\alpha$ is contaminated by the actual value of $\phi$. Thus it is desirable for these two subspaces to be orthogonal to each other. The formal definition is given in the next section.

\section{Adjusted orthogonality}

\subsection{Definition and results}

The definition of orthogonality in Section 4 seems surprising at first, because the vector subspaces $V_{F}$ and $V_{G}$ corresponding to two partitions $F$ and $G$ can never be orthogonal to each other. If $F$ is strictly orthogonal to $G$ then it is the projections of $V_{F}$ and $V_{G}$ onto the orthogonal complement of $V_{U}$ that are orthogonal to each other; equivalently, $X_{F}^{\top}\left(I_{e}-P_{U}\right) X_{G}=0$. It is tempting to say that $F$ and $G$ are orthogonal to each other after adjusting for $U$. More generally, $F$ is orthogonal to $G$ if and only if $X_{F}^{\top}\left(I_{e}-P_{F \vee G}\right) X_{G}=0$, which could be considered to be orthogonality after adjusting for $F \vee G$.

These comments lead to the notion of two partitions having adjusted orthogonality with respect to a third partition. For continuity with Section 7 , we define what it means for partitions $R$ and $C$ to have adjusted orthogonality with respect to partition $L$.

Definition Partitions $R$ and $C$ have adjusted orthogonality with respect to partition $L$ if $X_{R}^{\top}\left(I_{e}-P_{L}\right) X_{C}=0$.

Lemma 8.1 Partitions $R$ and $C$ have adjusted orthogonality with respect to partition $L$ if and only if

$$
N_{R L}\left(X_{L}^{\top} X_{L}\right)^{-1} N_{L C}=N_{R C}
$$


If $L$ is uniform, this is equivalent to

$$
N_{R L} N_{L C}=k_{L} N_{R C} .
$$

Theorem 8.2 Partitions $R$ and $C$ have adjusted orthogonality with respect to partition $L$ if and only if

$$
Q_{R} Q_{L} Q_{C}=Q_{R} Q_{C},
$$

where $Q_{F}=P_{F}-P_{U}$ for $F$ in $\{R, C, L\}$.

Proof Since $P_{F} P_{U}=P_{U} P_{F}=P_{U}$ for every partition $F$, equation (8.3) is equivalent to

$$
P_{R} P_{L} P_{C}=P_{R} P_{C} .
$$

Pre-multiplying both sides of this by $X_{R}^{\top}$ and post-multiplying both sides by $X_{C}$ gives $X_{R}^{\top} P_{L} X_{C}=X_{R}^{\top} X_{C}$. Conversely, pre-multiplying both sides of equation (8.1) by $X_{R}\left(X_{R}^{\top} X_{R}\right)^{-1}$ and post-multiplying both sides by $\left(X_{C}^{\top} X_{C}\right)^{-1} X_{C}^{\top}$ gives equation (8.4).

Note that this result does not require any of $R, C$ and $L$ to be uniform, nor does it need orthogonality between $R$ and $C$.

If $R \perp C$ and $R \wedge C$ is uniform then the entries in $N_{R C}$ are all the same. Then condition (8.2) becomes

$$
N_{R L} N_{L C} \text { is a scalar multiple of } J_{n_{R} n_{C}} \text {. }
$$

This has a clean combinatorial interpretation: the subset of letters in any row has a constant number of letters in common with every column (as usual, this needs a more precise explanation if the 'subset' is actually a multiset).

In the design in Figure 4, every row has three letters in common with every column. Therefore rows and columns have adjusted orthogonality with respect to letters.

Example 8.3 The design in Figure 12 is taken from [157], rearranged to show the partitions $R$ and $C$ as rows and columns. Here $R \wedge C$ is not uniform, because some parts of $R \wedge C$ have size one and others have size two. Moreover, $R$ is not orthogonal to $C$, because $N_{R C}=I_{5}+J_{5}$ when the rows and columns are labelled in the obvious way. However, $N_{R L} N_{L C}=3 N_{R C}$, so rows and columns have adjusted orthogonality with respect to letters even though rows are not orthogonal to columns. Expressed in another way, row $i$ has six letters in common with column $j$ if $i=j$ but only three letters in common if $i \neq j$. 


\begin{tabular}{|c|c|c|c|c|}
\hline$A \quad F$ & $D$ & $G$ & $J$ & $C$ \\
\hline$D$ & $B \quad G$ & $E$ & $H$ & $F$ \\
\hline$G$ & $E$ & $C \quad H$ & $A$ & $I$ \\
\hline$J$ & $H$ & $A$ & $D \quad I$ & $B$ \\
\hline$C$ & $F$ & $I$ & $B$ & $E \quad J$ \\
\hline
\end{tabular}

Figure 12: A design for 30 units, with partitions into rows, columns and letters

\subsection{Link with subspaces}

Some observations from Section 7 are gathered here.

Proposition 8.4 Let $R, C$ and $L$ be partitions of $\Omega$. Put $Q_{F}=P_{F}-P_{U}$ for $F$ in $\{R, C, L\}$. If $R \vee C=L \vee R=L \vee C=U$ then the following hold.

(i) $V_{L R} \perp V_{L C}$ if and only if $Q_{R} Q_{L} Q_{C}=0$.

(ii) If $V_{L R} \perp V_{L C}$ then $\operatorname{dim}\left(V_{L R}\right)+\operatorname{dim}\left(V_{L C}\right) \leq n_{L}-1$.

(iii) If $R \triangleright L, C \triangleright L$ and $V_{L R} \perp V_{L C}$ then $n_{R}+n_{C}-1 \leq n_{L}$.

(iv) $V_{R \vdash L} \perp V_{C \vdash L}$ if and only if $Q_{R}\left(I_{e}-Q_{L}\right) Q_{C}=0$.

(v) If $R \perp C$ then $Q_{R} Q_{C}=0$ and therefore $V_{L R} \perp V_{L C}$ if and only if $V_{R \vdash L} \perp V_{C \vdash L}$.

This shows that an alternative characterization of $R$ and $C$ having adjusted orthogonality with respect to $L$ is that the subspaces $V_{R \vdash L}$ and $V_{C \vdash L}$ are orthogonal to each other. Moreover, if, in addition, $R \perp C$, then the subspaces $V_{L R}$ and $V_{L C}$ are orthogonal to each other. Then part (iii) gives the following result.

Theorem 8.5 If $R \perp C, R \triangleright L, C \triangleright L$, and $R$ and $C$ have adjusted orthogonality with respect to $L$ then $n_{R}+n_{C}-1 \leq n_{L}$.

\subsection{A little history}

It seems that Potthoff [143] was the first to notice the importance of conditions like (8.2) for experiments with three partitions under the assumption of model (7.3). Preece also gave condition $(8.2)$ in $[145,147]$ in the situation where $R \perp C, L \triangleright R$ and $L \triangleright C$. Since they both assumed that $R \perp C$ and $R \wedge C=E$, they stated the condition in the form (8.5). 
Preece spent the year 1974-1975 in Australia: see [29]. At the end of his stay, he presented work on these designs at the Australian Conference on Combinatorial Mathematics in Adelaide: see [151]. This led Sterling and Wormald to give some constructions for such designs in [201] and Seberry and Street to take the ideas further in [187, 203].

Meanwhile, Eccleston and Russell had independently invented the idea of adjusted orthogonality, which they wrote as $R(L) \perp C(L)$ in [72], where they proved Lemma 8.1. They introduced the name 'adjusted orthogonality' in [73]. Papers [12, 19, 68, 69, 70, 74, 102, 184, 189, 190] followed, and the book [191] also had a section on adjusted orthogonality, but none of these mentioned the work of Preece.

Preece, Eccleston and Russell were all working in Statistics at universities in Sydney during the last four months of 1974. They met for discussions during this time, and Preece was external examiner for Russell's 1977 $\mathrm{PhD}$ thesis [183], whose Chapter 4 was devoted to adjusted orthogonality. Eccleston and Russell both report on Preece's very thorough reading of this $^{1}$. Nonetheless, when Preece cited [72] in his 1977 paper [154] it was only to say that this concept of orthogonality was not related to anyone else's. However, his article [157] for the Genstat Newsletter did use the phrase 'adjusted orthogonality', and explained it very clearly in the context of Example 8.3. I have not found an instance of his using the phrase 'adjusted orthogonality' in the mainstream literature before [164].

Bagchi listed authors who had constructed designs with adjusted orthogonality in [17], again with no mention of the many designs given by Preece in [147], and proved Theorem 8.5 in [18].

Eccleston and McGilchrist extended the ideas of [100] to three subspaces in [71] and applied their results to row-column designs. In particular, they proved that the average variance of estimators of differences like $\tau_{i}-\tau_{j}$ is bounded below by a known function of the average variances in the two block designs obtained when one of the partitions into rows and columns is ignored, and that this bound is achieved if rows and columns have adjusted orthogonality with respect to letters. Bagchi and Shah generalized this to a stronger notion of optimality in [21], but with no mention of [71].

Independently of Eccleston, Russell, and their co-authors, but building on the work of Preece in [145, 147, 151], Morgan and Uddin in [123] defined a block design for two non-interacting treatment factors $F$ and $G$ to be an orthogonal BIBD if $F \triangleright B, G \triangleright B$ and $k_{B} N_{F G}=N_{F B} N_{B G}$; this last condition is precisely adjusted orthogonality. The terminology OBIBD is also used in $[1,85,120]$; the first two of these state that the third condition

\footnotetext{
${ }^{1}$ Personal communications from JAE and KGR.
} 
is the same as adjusted orthogonality. Rees presented [1] at the British Combinatorial Conference in Sussex (2001).

\subsection{Adjusting for more than one partition}

In [72], Eccleston and Russell proposed a more general version of adjusted orthogonality. If $\mathcal{L}$ is a set of partitions of $\Omega$, put $V_{\mathcal{L}}=\sum_{L \in \mathcal{L}} V_{L}$, and let $P_{\mathcal{L}}$ be the matrix of orthogonal projection onto $V_{\mathcal{L}}$. By convention, $P_{\emptyset}=P_{\{U\}}=P_{U}=e^{-1} J_{e e}$. In the notation of [72], $R(\mathcal{L}) \perp C(\mathcal{L})$ if $\left(V_{R}+V_{\mathcal{L}}\right) \cap V_{\mathcal{L}}^{\perp}$ is orthogonal to $\left(V_{C}+V_{\mathcal{L}}\right) \cap V_{\mathcal{L}}^{\perp}$; equivalently,

$$
X_{R}^{\top}\left(I_{e}-P_{\mathcal{L}}\right) X_{C}=0
$$

In words, $R$ and $C$ have adjusted orthogonality with respect to $\mathcal{L}$.

In an important special case of this, $\mathcal{F}$ is a Tjur block structure and $\mathcal{L}=\mathcal{F} \backslash\{E\}$. Then $R$ and $C$ have adjusted orthogonality with respect to $\mathcal{L}$ if and only if the projections of $V_{R}$ and $V_{C}$ onto $W_{E}$ are orthogonal to each other, where $W_{E}$ is the subspace defined in Section 4.3.

\section{Adjusted balance}

\subsection{Terminology}

Section 8 discussed adjusted orthogonality, which is a possible relation between partitions $F$ and $G$ when everything is projected onto the orthogonal complement $V_{H}^{\perp}$ of $V_{H}$ for some other partition $H$. What happens when $F=G$ ? In this case, for clarity, we write $F=G=L$ and $H=B$.

Recall from Section 2 that $X_{L}^{\top} X_{L}$ is a diagonal matrix whose diagonal entries are the sizes of the parts of $L$. Thus $L$ is unform if and only if $X_{L}^{\top} X_{L}$ is completely symmetric. Some authors say that $L$ is balanced. Following on from Section 8 , it would be natural to say that $L$ has adjusted balance with respect to $B$ if $X_{L}^{\top}\left(I_{e}-P_{B}\right) X_{L}$ is completely symmetric. This is always true when $n_{L} \leq 2$, because the $n_{L} \times n_{L}$ matrix $X_{L}^{\top}\left(I_{e}-P_{B}\right) X_{L}$ is symmetric and has zero row-sums.

The requirement that $X_{L}^{\top}\left(I_{e}-P_{B}\right) X_{L}$ be completely symmetric is the main part of the definition of balance in Section 5.3. For consistency with Section 5, I shall continue to say 'is balanced with respect to' rather than 'has adjusted balance with respect to', but this discussion does show that the ideas in Sections 5 and 8 are closely related. However, there is one twist. In Section 5, we required $X_{L}^{\top}\left(I_{e}-P_{B}\right) X_{L}$ to be completely symmetric but not zero. Since $X_{L}^{\top} X_{L}$ itself cannot be zero, it is not unreasonable to include 'not zero' in the definition of balance. 


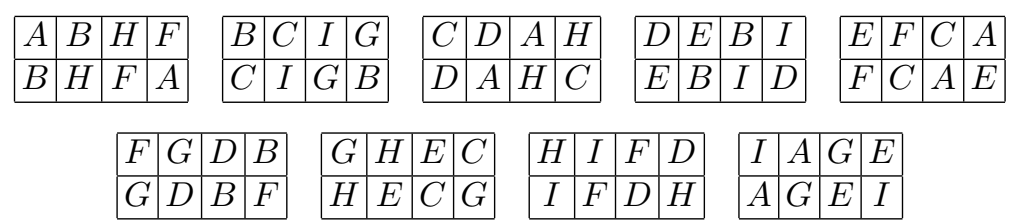

Figure 13: Design in Example 9.1: there are nine letters in nine blocks, each of which is a $2 \times 4$ rectangle

This twist shows a difference between orthogonality and balance. If $F \preccurlyeq G$ then $F \perp G$, so we regard refinement as a special case of orthogonality. However, if $F \preccurlyeq G$ then $X_{F}^{\top}\left(I_{e}-P_{G}\right) X_{F}=0$, so that $F$ is not balanced with respect to $G$, as noted in Section 5.2.

\subsection{General definition}

We can now define balance with respect to a set of partitions in a way that is analogous to the more general definition of adjusted orthogonality in Section 8.4.

Definition Let $\mathcal{G}$ be a set of partitions of $\Omega$, and let $L$ be a partition of $\Omega$. Then $L$ is balanced with respect to $\mathcal{G}$ if $X_{L}^{\top}\left(I_{e}-P_{\mathcal{G}}\right) X_{L}$ is completely symmetric but not zero.

It is immediate that $L$ cannot be balanced with respect to $\mathcal{G}$ if there is any $G$ in $\mathcal{G}$ for which $G \preccurlyeq L$. As in Section 8.4, an important special case occurs when $\mathcal{G}=\mathcal{F} \backslash\{E\}$ for some Tjur block structure $\mathcal{F}$. In this case, $L$ is balanced with respect to $\mathcal{G}$ if it is balanced with respect to $G$ for all in $\mathcal{G}$ and $X_{L}^{\top}\left(I_{e}-P_{\mathcal{G}}\right) X_{L}$ is not zero. The following example shows that it is possible to achieve balance with respect to $\mathcal{G}$ without having balance with respect to all $G$ in $\mathcal{G}$.

Example 9.1 The design in Figure 13 has 72 units, in nine blocks, each of which is a $2 \times 4$ rectangle. Nine letters have been allocated to the units. Denote by $B, R, C$ and $L$ the partitions into blocks, rows, columns and letters respectively. Then $P_{R, C, B}=P_{R}+P_{C}-P_{B}$ and so

$$
X_{L}^{\top}\left(I_{e}-P_{R, C, B}\right) X_{L}=X_{L}^{\top}\left(I_{e}-P_{C}\right) X_{L}-X_{L}^{\top}\left(P_{R}-P_{B}\right) X_{L} .
$$

In this design, the two rows in each block have exactly the same set of letters, with the result that $X_{L}^{\top}\left(P_{R}-P_{B}\right) X_{L}=0$. It follows that 


\begin{tabular}{|c|c|c|c|c|c|}
\hline$D$ & $E$ & $F$ & $G$ & $H$ & $I$ \\
\hline$H$ & $I$ & $G$ & $C$ & $A$ & $B$ \\
\hline$C$ & $A$ & $B$ & $E$ & $F$ & $D$ \\
\hline$B$ & $H$ & $E$ & $I$ & $C$ & $F$ \\
\hline$F$ & $C$ & $I$ & $D$ & $G$ & $A$ \\
\hline$G$ & $D$ & $A$ & $B$ & $E$ & $H$ \\
\hline
\end{tabular}

Figure 14: Row-column design in which letters are balanced with respect to $\{R, C\}$ while rows and columns have adjusted orthogonality with respect to letters

$X_{L}^{\top}\left(I_{e}-P_{R, C, B}\right) X_{L}=X_{L}^{\top}\left(I_{e}-P_{C}\right) X_{L}$, which is completely symmetric and nonzero, because $L \triangleright C$. Hence $L$ is balanced with respect to $\{R, C, B\}$, even though it is not balanced with respect to either $R$ or $B$. In fact, in this example $P_{R, C, B}=P_{R, C}$ because $R \prec B$, and so $L$ is also balanced with respect to $\{R, C\}$.

\subsection{Balance with respect to a pair of partitions}

The most common use of this more general concept of balance is for the case that $\mathcal{G}=\{R, C\}$ and $R \vee C=U$, so that we are interested in the projection of the data onto $\left(V_{R}+V_{C}\right)^{\perp}$. Let $Q_{R C}$ be the matrix of orthogonal projection onto $\left(V_{R}+V_{C}\right) \cap V_{U}^{\perp}$; and put $Q_{R}=P_{R}-P_{U}$ and $Q_{C}=P_{C}-P_{U}$. Then $L$ is balanced with respect to the pair $\{R, C\}$ if $X_{L}^{\top}\left(I_{e}-Q_{R C}-P_{U}\right) X_{L}$ is completely symmetric but not zero.

This terminology is consistent with that in [25], but it is not ideal, because ' $L$ is balanced with respect to $R$ and $C$ ' might mean ' $L \triangleright R$ and $L \gg C$ ' or it might mean ' $L$ is balanced with respect to $\{R, C\}$ '. In [151], Preece calls it ' $L$ has overall total balance with respect to the rest of the design'; in later papers this becomes ' $L$ is fully balanced ...'.

If $R \gg C$ then equation (7.1) gives $X_{L}^{\top} Q_{R C} X_{L}$. If, additionally, either $L \perp R$ or $L \perp C$ then $X_{L}^{\top} Q_{R C} X_{L}$ is a scalar multiple of one of $X_{L}^{\top} Q_{R} X_{L}$ and $X_{L}^{\top} Q_{C} X_{L}$, so the properties of $X_{L}^{\top} Q_{R C} X_{L}$ follow from those of the binary relations between $L$ and $R$ and between $L$ and $C$.

If $R \perp C$ then $Q_{R C}=Q_{R}+Q_{C}$ and so the properties of $X_{L}^{\top} Q_{R C} X_{L}$ are derivable from those of $X_{L}^{\top} Q_{R} X_{L}$ and $X_{L}^{\top} Q_{C} X_{L}$ considered together. It may be possible for their sum to be completely symmetric even though neither is. For example, in a resolvable BIBD in which $n_{B}=2 k_{B}$ it may be possible to allocate the letters to the cells of a $k_{B} \times k_{B}$ square in such a way that the rows form half of the original blocks and the columns form the others. Figure 14 shows an example with $n_{L}=9, n_{B}=12$ and $k_{B}=6$ 
(the blocks are the complements of those in Figure 1). In this design, it is also true that rows and columns have adjusted orthogonality with respect to letters. Many more examples are given in [113, 134]. Preece found 345 species (that is, merging isomorphism classes obtainable from each other by interchanging rows and columns) of designs with these parameters and properties in [149, 153], while McSorley and Phillips completed the enumeration to 348 by a computer search reported in [117].

\subsection{Three-way balance with pairwise balance}

Suppose that $C \backslash R$. Equation (7.1) shows that the condition for $L$ to be balanced with respect to $\{R, C\}$ is that the matrix

$$
\begin{array}{r}
(1-\lambda) X_{L}^{\top} X_{L}-(1-\lambda) X_{L}^{\top} P_{U} X_{L}-(1-\lambda) X_{L}^{\top} Q_{R} X_{L}-X_{L}^{\top} Q_{C} X_{L} \\
+X_{L}^{\top} Q_{R} Q_{C} X_{L}+X_{L}^{\top} Q_{C} Q_{R} X_{L}-X_{L}^{\top} Q_{R} Q_{C} Q_{R} X_{L}
\end{array}
$$

is completely symmetric but not zero. If $L>R$ and $L \triangleright C$ then the first four terms are completely symmetric, and so the first part of this condition becomes

$$
\begin{array}{r}
k_{R}\left(N_{L R} N_{R C} N_{C L}+N_{L C} N_{C R} N_{R L}\right)-N_{L R} N_{R C} N_{C R} N_{R L} \\
\text { is completely symmetric. }
\end{array}
$$

Condition (9.1) is given explicitly in [151].

If, in addition, $C$ and $L$ have adjusted orthogonality with respect to $R$, then Lemma 8.1 shows that $N_{C R} N_{R L}$ is a scalar multiple of $N_{C L}$. Therefore the matrix in (9.1) is a multiple of $N_{L C} N_{C L}$, which is completely symmetric because $L \backslash C$. Thus adjusted orthogonality gives a special case of this type of three-way balance.

In [147], Preece gave 59 designs with three partitions $R, C$ and $L$ satisfying $n_{R}=n_{C}<n_{L}, R \triangleright L, C \triangleright L, R \bowtie C$ and $N_{R L} N_{L C}=$ $k_{L} N_{R C}$. It follows that $R$ and $C$ have adjusted orthogonality with respect to letters, that $R$ is balanced with respect to $\{C, L\}$, and that $C$ is balanced with respect to $\{R, L\}$. Figure 15 shows an example. Street generalized his constructions in [203] to give infinite families of designs, and widened the scope by relaxing the final condition to allow $N_{R L} N_{L C}$ to be any completely symmetric matrix. Agrawal and Sharma gave further designs of this type in [10].

On the other hand, if $n_{L} \leq n_{C}=n_{R}$ then equation (7.2) gives the following (ignoring the possibility that $X_{L}^{\top}\left(I_{e}-Q_{R C}-P_{U}\right) X_{L}$ might be zero). 


\begin{tabular}{|c|c|c|c|c|c|c|c|}
\hline & $L$ & $I$ & $F$ & $J$ & $G$ & $D$ & $A$ \\
\hline$E$ & & $M$ & $J$ & $G$ & $K$ & $A$ & $B$ \\
\hline$B$ & $F$ & & $N$ & $K$ & $A$ & $L$ & $C$ \\
\hline$M$ & $C$ & $G$ & & $H$ & $L$ & $B$ & $D$ \\
\hline$C$ & $N$ & $D$ & $A$ & & $I$ & $M$ & $E$ \\
\hline$N$ & $D$ & $H$ & $E$ & $B$ & & $J$ & $F$ \\
\hline$K$ & $H$ & $E$ & $I$ & $F$ & $C$ & & $G$ \\
\hline$H$ & $I$ & $J$ & $K$ & $L$ & $M$ & $N$ & \\
\hline
\end{tabular}

Figure 15: A design for 56 units, with partitions into rows, columns and letters: blank cells indicate empty row-column intersections

Proposition 9.2 Suppose that $R \bowtie C, L \triangleright R$ and $L \triangleright C$. Then $L$ is balanced with respect to $\{R, C\}$ if and only if

$N_{L R} N_{R C} N_{C L}+N_{L C} N_{C R} N_{R L}$ is completely symmetric.

A stronger condition is

$$
N_{L R} N_{R C} \text { is a linear combination of } N_{L C} \text { and } J_{n_{L} n_{C}} \text {. }
$$

Proofs of the following are in [25].

Proposition 9.3 If $R \bowtie C, L \triangleright R$ and $L \triangleright C$ then the following hold.

(i) Condition (9.3) implies condition (9.2).

(ii) If $n_{L}=n_{C}$ and condition (9.2) is satisfied for the ordered triple $(L, R, C)$ then it is satisfied for any permutation of $\{L, R, C\}$.

(iii) If $n_{L}=n_{C}$ and condition (9.3) is satisfied for the ordered triple $(L, R, C)$ then it is satisfied for any permutation of $\{L, R, C\}$.

Figure 16 shows two designs for 28 units with three partitions having seven parts of size four. In both designs, all the pairwise relations between partitions are strict balance in both directions. However, in the design in Figure 16(a), taken from [98], none of these partitions is balanced with respect to the other pair, whereas in the design in Figure 16(b), taken from [145], every one of these partitions is balanced with respect to the other pair. 


\begin{tabular}{|c|c|c|c|c|c|c|}
\hline$A$ & $B$ & $C$ & & $D$ & & \\
\hline$E$ & $A$ & & & & $B$ & $F$ \\
\hline & $D$ & $A$ & $F$ & & & $G$ \\
\hline & $C$ & & $E$ & $A$ & $G$ & \\
\hline$B$ & & $G$ & $D$ & & $E$ & \\
\hline$F$ & & & $C$ & $G$ & & $B$ \\
\hline & & $E$ & & $F$ & $D$ & $C$ \\
\hline
\end{tabular}

(a)

\begin{tabular}{|c|c|c|c|c|c|c|}
\hline$A$ & $E$ & $B$ & & $C$ & & \\
\hline & $B$ & $F$ & $C$ & & $D$ & \\
\hline & & $C$ & $G$ & $D$ & & $E$ \\
\hline$F$ & & & $D$ & $A$ & $E$ & \\
\hline & $G$ & & & $E$ & $B$ & $F$ \\
\hline$G$ & & $A$ & & & $F$ & $C$ \\
\hline$D$ & $A$ & & $B$ & & & $G$ \\
\hline
\end{tabular}

(b)

Figure 16: Two designs for 28 units, with partitions into rows, columns and letters: blank cells indicate empty row-column intersections

\section{Three partitions}

\subsection{Supreme sets of uniform partitions}

For simplicity, from now on we confine our interest to uniform partitions on $\Omega$. The following definition is taken from [32].

Definition A set $\mathcal{F}$ of partitions on $\Omega$ is supreme if $\mathcal{F} \cup\{U\}$ is closed under taking suprema.

We shall examine supreme sets $\mathcal{F}$ of uniform non-trivial partitions of $\Omega$ with the property that if $F$ and $G$ are in $\mathcal{F}$ then at least one of the following holds: (i) $F$ is orthogonal to $G$ (this includes $F \prec G$ and $G \prec F$ ); (ii) at least one of $F$ and $G$ is strictly balanced with respect to the other.

Pearce and co-authors discussed sets of (usually) uniform partitions in $[98,134]$ and introduced notation for various binary relations between them. Preece augmented the notation in [145] and displayed the relations in a matrix whose rows and columns are labelled by the partitions. The diagonal is empty. If $F \neq G$ then the $(F, G)$-entry is $O$ (for 'orthogonal') if $F$ is strictly orthogonal to $G$ and $F \wedge G$ is uniform; it is $T$ (for 'total balance') if $F$ is binary balanced with respect to $G$ but not orthogonal to $G$; it is $T^{\prime}$ (with the connotation that the transpose indicates the reverse relationship) if $F$ is neither orthogonal nor binary balanced with respect to $G$ but $G$ is binary balanced with respect to $F$.

For example, denote by $R, C$ and $L$ the partitions into rows, columns and letters in Figures 3 and 4 . In this notation, the matrices for these 
row-column designs are

$$
\left.\begin{array}{c}
R \\
C \\
L
\end{array}\left[\begin{array}{ccc}
R & C & L \\
- & O & O \\
O & - & T \\
O & T & -
\end{array}\right] \quad \text { and } \quad \begin{array}{c}
R \\
C \\
\end{array} \quad \begin{array}{ccc}
R & C & L \\
- & O & T \\
O & - & T \\
T^{\prime} & T^{\prime} & -
\end{array}\right]
$$

respectively.

In [32], Bailey and Cameron convey the same information by showing each partition as a vertex of a directed graph and labelling the edges with symbols to show the relationships. Here we shall simply use the symbols $\perp, \perp, \prec, \succ, \triangleleft, \triangleright$ and $\bowtie$.

\subsection{Two partitions}

Suppose that $\mathcal{F}=\{F, G\}$, where $F$ and $G$ are distinct uniform nontrivial partitions. If $\mathcal{F}$ is supreme, then, up to renaming, either $F \prec G$ or $F \vee G=U$. The first case gives a poset block structure with $\mathcal{P}=\{1,2,3\}$ and $3 \sqsubset 2 \sqsubset 1$, where $F=F_{\{1,2\}}$ and $G=F_{\{1\}}$.

If $F \vee G=U$ and $F \perp G$ then $F \perp G$ and all parts of $F \wedge G$ have the same size, by Proposition 4.1. Then $F$ and $G$ can be regarded as the partitions of a rectangle into rows and columns, with each row-column intersection containing the same number of units. If $F \wedge G=E$ this is the poset block structure defined by $\mathcal{P}=\{1,2\}$ with trivial partial order.

If $F \vee G=U$ but $F$ is not orthogonal to $G$ then, up to renaming, $F \triangleright G$. Altogether, we have these possible structures.

A.1 The poset block structure defined by $\mathcal{P}=\{1,2,3\}$ and $3 \sqsubset 2 \sqsubset 1$, with $\mathcal{F}=\left\{F_{\{1\}}, F_{\{1,2\}}\right\}$.

A.2 The poset block structure defined by $\mathcal{P}=\{1,2\}$ with trivial partial order, with $\mathcal{F}=\left\{F_{\{1\}}, F_{\{2\}}\right\}$.

A.3 The poset block structure defined by $\mathcal{P}=\{1,2,3\}, 3 \sqsubset 1$ and $3 \sqsubset 2$, with $\mathcal{F}=\left\{F_{\{1\}}, F_{\{2\}}\right\}$.

A.4 $F \triangleright G$ but $G$ is not balanced with respect to $F$, so that $n_{F}<n_{G}$.

A.5 $F \bowtie G$, so that $n_{F}=n_{G}$.

\subsection{Three partitions: three orthogonal relations}

Suppose that $\mathcal{F}=\{F, G, H\}$, where $F, G$ and $H$ are distinct uniform non-trivial partitions and all the pairwise relations are orthogonality. If $\mathcal{F}$ is supreme but no supremum is $U$ then, up to renaming, either $F \prec$ 
$G \prec H$ or $F \vee G=H$. The first case gives a poset block structure with $\mathcal{P}=\{1,2,3,4\}$ and $4 \sqsubset 3 \sqsubset 2 \sqsubset 1$, where $F=F_{\{1,2,3\}}, G=F_{\{1,2\}}$ and $H=F_{\{1\}}$. In the second case, the parts of $H$ can be considered as rectangles, each of which is partitioned into rows and columns as in structures A.2 or A.3: the first of these gives Example 4.4. Because $F, G$ and $H$ are all uniform, Proposition 4.1 shows that $F \wedge G$ is uniform.

If only one supremum is $U$, suppose that it is $G \vee H$. Then $F \prec G$ and $F \prec H$, so $F \preccurlyeq G \wedge H$. This gives two cases: $F=G \wedge H$ and $F \prec G \wedge H$. If precisely two suprema are $U$, suppose that they are $F \vee H$ and $G \vee H$. Then either $F \prec G$ or $G \prec F$, and we obtain a poset block structure like the one in Example 4.5. If all three suprema are $U$ then we have an orthogonal array of strength two. This is not necessarily derived from a poset block structure: for example, $F, G$ and $H$ could be three of the partitions in Figure 7.

This gives the following possible structures.

B.1 The poset block structure defined by $\mathcal{P}=\{1,2,3,4\}$ and $4 \sqsubset 3 \sqsubset$ $2 \sqsubset 1$, with $\mathcal{F}=\left\{F_{\{1\}}, F_{\{1,2\}}, F_{\{1,2,3\}}\right\}$.

B. 2 The poset block structure defined by $\mathcal{P}=\{1,2,3\}, 2 \sqsubset 1$ and $3 \sqsubset 1$, with $\mathcal{F}=\left\{F_{\{1\}}, F_{\{1,2\}}, F_{\{1,3\}}\right\}$.

B.3 The poset block structure defined by $\mathcal{P}=\{1,2,3,4\}, 4 \sqsubset 3 \sqsubset 1$ and $4 \sqsubset 2 \sqsubset 1$, with $\mathcal{F}=\left\{F_{\{1\}}, F_{\{1,2\}}, F_{\{1,3\}}\right\}$.

B. 4 The poset block structure defined by $\mathcal{P}=\{1,2,3\}, 3 \sqsubset 1$ and $3 \sqsubset 2$, with $\mathcal{F}=\left\{F_{\{1\}}, F_{\{2\}}, F_{\{1,2\}}\right\}$.

B.5 The poset block structure defined by $\mathcal{P}=\{1,2,3,4\}, 4 \sqsubset 3 \sqsubset 1$ and $4 \sqsubset 3 \sqsubset 2$, with $\mathcal{F}=\left\{F_{\{1\}}, F_{\{2\}}, F_{\{1,2,3\}}\right\}$.

B.6 The poset block structure defined by $\mathcal{P}=\{1,2,3\}$ and $2 \sqsubset 1$, with $\mathcal{F}=\left\{F_{\{1\}}, F_{\{1,2\}}, F_{\{3\}}\right\}$.

B.7 An orthogonal array of strength two containing three partitions.

\subsection{Three partitions: two relations of orthogonality and one of balance}

Suppose that $\mathcal{F}=\{F, G, H\}$, where $F, G$ and $H$ are distinct uniform non-trivial partitions, $\mathcal{F}$ is supreme, $F \triangleright G, F \perp H$ and $G \perp H$. Since $F \vee G=U$, we cannot have $H \succ F$ and $H \succ G$, because that implies that $H \succcurlyeq F \vee G$.

If $H \prec F$ and $H \prec G$ then $E \neq H \preccurlyeq F \wedge G$ and so the relationship between $F$ and $G$ cannot be binary. If it is generalized binary then the 
parts of $F \wedge G$ have size differing by one, but this cannot happen, because $H$ is uniform.

Because $F$ and $G$ are not related by $\prec, H$ cannot be finer than one and coarser than the other. If $H \perp G$ then every part of $H$ meets every part of $G$ in $k_{H} k_{G} / e$ units. If, in addition, $H \prec F$, then every part of $F$ meets every part of $G$ in a constant number of units, so $F \perp G$, contrary to the assumptions. We get a similar contradiction if $H \perp F$ and $H \prec G$.

If $G \prec H$ and $H \perp F$ then the parts of $G$ are grouped into parts of $H$, each of which has $m$ units in common with every part of $F$, where $m=$ $k_{H} k_{F} / e$. With the parts of $F$ and $G$ considered as letters and blocks respectively, this is called an $m$-resolvable design. When $m=1$, this is just a resolvable design, as in Example 1.3. Bose proved a generalization of Fisher's inequality in [47]: in such a design, $n_{G}-n_{H} \geq n_{F}-1$. This can be proved by using parts (i), (ii) and (v) of Theorem 7.1 and noting that $V_{G-F} \geq V_{H} \cap V_{U}^{\perp}$. Hence $G$ cannot be balanced with respect to $F$.

On the other hand, if $F \prec H$ and $H \perp G$ then a short counting argument shows that the average concurrence (in parts of $G$ ) of parts of $F$ within the same part of $H$ is $k_{F}\left(k_{G}-n_{H}\right) /\left(n_{F}-n_{H}\right)$ while the average concurrence between other parts of $F$ is $k_{F} k_{G} / n_{F}$. These cannot be the same unless $n_{F}=k_{G}$, which is impossible when $F \triangleright G$.

In the remaining case, $H \perp F$ and $H \perp G$. To aid thought, rename $H$ as $R, G$ as $C$ and $F$ as $L$, so that the parts of $R$ and $C$ are the rows and columns of a rectangle. Either $R \wedge C=E$ or $E \prec R \wedge C$. Moreover, $L \perp R$, $L \triangleright C$, and either $L \bowtie C$ or not.

First suppose that $R \wedge C=E$. If $n_{L}=n_{C}$ then Hall's Marriage Theorem shows that any strictly balanced block design can have the letters allocated to the units in the rectangle in such a way that the columns are blocks and every letter comes exactly once in each row. When $n_{R}<n_{L}$ this is called a Youden square. Figure 3 shows an example.

The usage of generalized Youden design in [4, 192, 204] relaxes the condition that $n_{L}=n_{C}$. There seems little to lose if the condition that $n_{R}<n_{L}$ is also relaxed. For example, putting two copies of the design in Figure 3 side by side and putting two Latin squares of order 7 underneath them gives a design with $n_{R}=11, n_{C}=14$ and $n_{L}=7$ in which $R \perp C$, $R \perp L, R \wedge C=R \wedge L=E$ and $L \triangleright C$. This is not the only method of construction. For example, start with the design in Figure 1, glue the blocks together to make a $3 \times 12$ rectangle, make two further copies by permuting whole rows by a cycle of order 3 , and then place all of these side by side to obtain a design with $n_{R}=3, n_{C}=36$ and $n_{L}=9$.

Finally suppose that $E \prec R \wedge C$. Because $R \wedge C$ is not in $\mathcal{F}$, there is no constraint on the relationship between $L$ and $R \wedge C$. For example, in the designs in Figures 17(a) and 18 letters are balanced with respect to 


\begin{tabular}{|l|l|l|l|l|l|l|}
\hline$A$ & $B$ & $C$ & $D$ & $E$ & $F$ & $G$ \\
$B$ & $C$ & $D$ & $E$ & $F$ & $G$ & $A$ \\
\hline$G$ & $A$ & $B$ & $C$ & $D$ & $E$ & $F$ \\
$C$ & $D$ & $E$ & $F$ & $G$ & $A$ & $B$ \\
\hline$D$ & $E$ & $F$ & $G$ & $A$ & $B$ & $C$ \\
$F$ & $G$ & $A$ & $B$ & $C$ & $D$ & $E$ \\
\hline
\end{tabular}

(a)

\begin{tabular}{|l|l|l|l|l|l|l|}
\hline$A$ & $B$ & $C$ & $D$ & $E$ & $F$ & $G$ \\
$B$ & $C$ & $D$ & $E$ & $F$ & $G$ & $A$ \\
\hline$C$ & $D$ & $E$ & $F$ & $G$ & $A$ & $B$ \\
$D$ & $E$ & $F$ & $G$ & $A$ & $B$ & $C$ \\
\hline$E$ & $F$ & $G$ & $A$ & $B$ & $C$ & $D$ \\
$F$ & $G$ & $A$ & $B$ & $C$ & $D$ & $E$ \\
\hline
\end{tabular}

(b)

Figure 17: Two $3 \times 7$ row-column designs in which letters and columns are strictly balanced with respect to each other and strictly orthogonal to rows

\begin{tabular}{|l|l|l|l|l|l|l|l|l|l|l|l|l|l|l|}
\hline$A$ & $A$ & $A$ & $A$ & $A$ & $B$ & $C$ & $D$ & $E$ & $F$ & $C$ & $D$ & $E$ & $F$ & $B$ \\
$B$ & $C$ & $D$ & $E$ & $F$ & $C$ & $D$ & $E$ & $F$ & $B$ & $E$ & $F$ & $B$ & $C$ & $D$ \\
\hline$C$ & $D$ & $E$ & $F$ & $B$ & $A$ & $A$ & $A$ & $A$ & $A$ & $D$ & $E$ & $F$ & $B$ & $C$ \\
$D$ & $E$ & $F$ & $B$ & $C$ & $E$ & $F$ & $B$ & $C$ & $D$ & $F$ & $B$ & $C$ & $D$ & $E$ \\
\hline
\end{tabular}

Figure 18: A $2 \times 15$ row-column design in which letters are strictly balanced with respect columns, but not vice versa, and both are strictly orthogonal to rows

$R \wedge C$, whereas in the designs in Figures $17(\mathrm{~b})$ and 19 they are not. As with orthogonal arrays of strength two, we ignore this distinction here. In both designs in Figure 17, and in the design in Figure 19, $L \bowtie C$. This is not true in the design in Figure 18.

Thus we have the following possible structures.

C.1 An $m$-resolved strictly balanced block design, for $m \geq 1$, with partitions into letters $(L)$, blocks $(B)$ and districts $(D)$ as in Example 1.3. Then $\mathcal{F}=\{L, B, D\}$. See Section 15 .

C.2 A Youden square: a row-column design in which $R \wedge C=C \wedge L=E$, letters and columns are both strictly orthogonal to rows and $L \bowtie C$, or the generalization that does not demand that $C \wedge L=E$. Here $\mathcal{F}=\{R, C, L\}$. See Section 11 .

C.3 A row-column design in which $R \wedge C=E$, rows are strictly orthogonal to both columns and letters, $L \triangleright C$ and $n_{C}>n_{L}$. Here $\mathcal{F}=\{R, C, L\}$.

C.4 A row-column design in which $R \wedge C \neq E$, rows are strictly orthogonal to both columns and letters, and $L \bowtie C$. Here $\mathcal{F}=\{R, C, L\}$. 


\begin{tabular}{|l|l|l|l|l|l|l|l|l|l|l|l|l|l|l|l|}
\hline$B$ & $A$ & $D$ & $C$ & $F$ & $E$ & $H$ & $G$ & $J$ & $I$ & $L$ & $K$ & $N$ & $M$ & $P$ & $O$ \\
$E$ & $F$ & $G$ & $H$ & $A$ & $B$ & $C$ & $D$ & $M$ & $N$ & $O$ & $P$ & $I$ & $J$ & $K$ & $L$ \\
\hline$C$ & $D$ & $A$ & $B$ & $G$ & $H$ & $E$ & $F$ & $K$ & $L$ & $I$ & $J$ & $P$ & $O$ & $N$ & $M$ \\
$I$ & $J$ & $K$ & $L$ & $M$ & $N$ & $O$ & $P$ & $E$ & $F$ & $G$ & $H$ & $A$ & $B$ & $C$ & $D$ \\
\hline$D$ & $C$ & $B$ & $A$ & $H$ & $G$ & $F$ & $E$ & $L$ & $K$ & $J$ & $I$ & $O$ & $P$ & $M$ & $N$ \\
$M$ & $N$ & $O$ & $P$ & $I$ & $J$ & $K$ & $L$ & $A$ & $B$ & $C$ & $D$ & $E$ & $F$ & $G$ & $H$ \\
\hline
\end{tabular}

Figure 19: A $3 \times 16$ row-column design in which letters and columns are strictly balanced with respect to each other and strictly orthogonal to rows

C.5 A row-column design in which $R \wedge C \neq E$, rows are strictly orthogonal to both columns and letters, $L \triangleright C$ and $n_{C}>n_{L}$. Here $\mathcal{F}=\{R, C, L\}$.

\subsection{Three partitions: one relation of orthogonality and two of balance}

Suppose that $\mathcal{F}=\{F, G, H\}$ where $F \perp G$ and the relation between $H$ and each of the others is non-orthogonal strict balance in at least one direction. If $\mathcal{F}$ is supreme then, up to renaming, either $F \prec G$ or $F \perp G$.

If $F \prec G$ it is convenient to rename $F, G$ and $H$ as $B, D$ and $L$ respectively, as in Example 1.3. If $L$ is strictly balanced with respect to both $B$ and $D$ then $\mathcal{F}$ is a nested balanced block design. If, in addition, $L \wedge D=E$, then $\mathcal{F}$ is a nested BIBD: see Section 12. If $L \wedge D \neq E$ then $L \wedge B$ may or may not be $E$. Possible linear models, and consequent methods of estimation, are given in Section 6.2.1.

In this case, Fisher's inequality gives $n_{B}>n_{D} \geq n_{L}$. Thus it is possible to have $L \bowtie D$ but not to have $L \bowtie B$.

On the other hand, if $n_{L} \geq n_{B}>n_{D}$ then it may be possible to have both $B \triangleright L$ and $D \triangleright L$. Figure 20 shows an example. Then every pair of blocks concur in at least one part of $L$, and so $L \wedge D \neq E$. In Figure 20, $n_{B}=n_{L}$ and so $B \bowtie L$. If each letter in Figure 20 is replaced by two letters, so that $k_{B}=6$ and $n_{L}=8$, then we have an example with $n_{L}>n_{B}>n_{D}$.

The third possibility is $n_{B}>n_{L}>n_{D}$ with $D \triangleright L \triangleright B$. Figure 21 shows such a design with $n_{D}=2, n_{L}=6$ and $n_{B}=10$.

If $F \perp G$ then, for clarity, rename $F, G$ and $H$ as $R, C$ and $L$ so that the parts of $R$ and $C$ can be visualized as the rows and columns of a rectangle. It may or may not be the case that $R \wedge C=E$.

Since $k_{R}=n_{C} k_{R \wedge C}$, if $n_{L}$ divides $n_{C}$ then the relationship between $L$ 


$$
\text { 圆闾圆周 }
$$

Figure 20: A nested block design in which blocks (shown as columns) and districts (shown as rectangles) are both strictly balanced with respect to letters

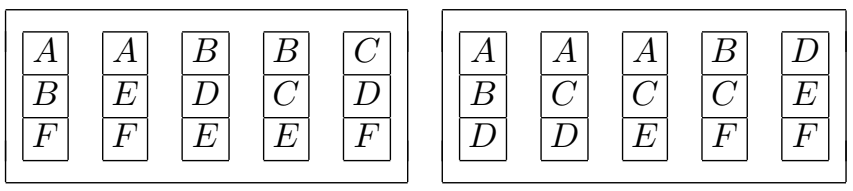

Figure 21: A nested block design in which districts (shown as rectangles) are strictly balanced with respect to letters and letters are strictly balanced with respect to blocks (shown as columns)

and $R$ cannot be generalized binary unless $L \perp R$, which is contrary to our assumptions. Hence $n_{L} \neq n_{C}$. Similarly, $n_{L} \neq n_{R}$.

Since both relations involving $L$ are not orthogonality, Section 7.2 shows that we need to consider adjusted orthogonality. If $L \triangleright R$ then $V_{L R}=V_{L} \cap V_{U}^{\perp}$, so Proposition 8.4 shows that it is impossible for $R$ and $C$ to have adjusted orthogonality with respect to $L$. The same is true if $L \triangleright C$. Thus adjusted orthogonality requires $R \triangleright L, C \triangleright L$ and $n_{R}+n_{C}-1 \leq n_{L}$.

\begin{tabular}{|l|l|l|l|l|l|}
\hline$A$ & $B$ & $C$ & $D$ & $A$ & $B$ \\
\hline$B$ & $C$ & $D$ & $A$ & $A$ & $D$ \\
\hline$C$ & $D$ & $A$ & $B$ & $B$ & $C$ \\
\hline$D$ & $A$ & $B$ & $C$ & $C$ & $A$ \\
\hline$A$ & $B$ & $C$ & $D$ & $C$ & $D$ \\
\hline$B$ & $C$ & $D$ & $A$ & $D$ & $B$ \\
\hline
\end{tabular}

(a)

\begin{tabular}{|l|l|l|l|l|l|}
\hline$A$ & $D$ & $C$ & $B$ & $A$ & $D$ \\
\hline$B$ & $B$ & $D$ & $A$ & $C$ & $C$ \\
\hline$D$ & $C$ & $B$ & $C$ & $D$ & $A$ \\
\hline$C$ & $A$ & $D$ & $A$ & $B$ & $B$ \\
\hline$C$ & $C$ & $B$ & $D$ & $A$ & $A$ \\
\hline$B$ & $D$ & $A$ & $D$ & $B$ & $C$ \\
\hline
\end{tabular}

(b)

\begin{tabular}{|l|l|l|l|l|l|}
\hline$A$ & $B$ & $C$ & $D$ & $A$ & $B$ \\
\hline$B$ & $A$ & $D$ & $C$ & $B$ & $C$ \\
\hline$C$ & $D$ & $A$ & $B$ & $C$ & $D$ \\
\hline$D$ & $C$ & $B$ & $A$ & $D$ & $A$ \\
\hline$A$ & $C$ & $B$ & $D$ & $A$ & $C$ \\
\hline$C$ & $B$ & $D$ & $A$ & $B$ & $D$ \\
\hline
\end{tabular}

(c)

Figure 22: Three non-isomorphic row-column designs in which letters are strictly balanced with respect to both rows and columns 


\begin{tabular}{|c|c|c|c|c|c|}
\hline$A$ & $H$ & $G$ & $F$ & $J$ & $E$ \\
\hline$F$ & $A$ & $I$ & $C$ & $H$ & $D$ \\
\hline$I$ & $B$ & $J$ & $G$ & $D$ & $A$ \\
\hline$G$ & $F$ & $B$ & $D$ & $E$ & $C$ \\
\hline$E$ & $J$ & $C$ & $H$ & $I$ & $B$ \\
\hline
\end{tabular}

Figure 23: Combinatorial design in which rows and columns are both balanced with respect to letters but do not have adjusted orthogonality with respect to letters

\begin{tabular}{|l|l|l|l|l|l|l|l|l|l|l|l|l|l|l|}
\hline$C$ & $A$ & $B$ & $B$ & $A$ & $C$ & $F$ & $D$ & $E$ & $B$ & $A$ & $C$ & $D$ & $E$ & $F$ \\
\hline$E$ & $F$ & $C$ & $D$ & $F$ & $A$ & $E$ & $C$ & $B$ & $D$ & $F$ & $A$ & $E$ & $C$ & $B$ \\
\hline$D$ & $E$ & $A$ & $F$ & $B$ & $E$ & $C$ & $A$ & $D$ & $F$ & $E$ & $B$ & $C$ & $A$ & $D$ \\
\hline$F$ & $B$ & $D$ & $E$ & $C$ & $D$ & $B$ & $F$ & $A$ & $C$ & $D$ & $E$ & $B$ & $F$ & $A$ \\
\hline
\end{tabular}

Figure 24: A $4 \times 15$ row-column design in which rows are strictly balanced with respect to letters, which are in turn strictly balanced with respect to columns

If $n_{L}<n_{R}$ and $n_{L}<n_{C}$ then it may be possible to have $L \triangleright R$ and $L \triangleright C$. Figure 22 shows three non-isomorphic such designs with $R \wedge C=E, n_{L}=4$ and $n_{R}=n_{C}=6$. The one in Figure 22(a) was given in [136]; that in Figure 22(b), which was used for an experiment on strawberries, was given in [134]. A complete enumeration of species for such designs of this size is in [166], which was presented by Côté at the BCC in Surrey (1991). In such a design, every vector in $V_{L} \cap V_{U}^{\perp}$ makes the same angle with $\left(V_{R}+V_{C}\right)^{\perp}$, with the result that estimates of all differences like $\tau_{i}-\tau_{j}$ have the same variance. Such a design cannot have adjusted orthogonality. Some authors include designs like this in generalized Youden designs if $R \wedge C=E$ : see [108, 109].

If $n_{L}>n_{R}$ and $n_{L}>n_{C}$ then it may be possible to have $R \triangleright L$ and $C \triangleright L$. This occurs in the design in Figure 4, which does have adjusted orthogonality. Figure 23 shows a design with all the same properties except that it does not have adjusted orthogonality. These designs are called double arrays in $[116,118]$.

The third possibility is, up to interchanging rows and colunms, that $n_{R}<n_{L}<n_{C}$ and $R \triangleright L$ and $L \triangleright C$. Again, adjusted orthogonality is impossible. Figure 24 shows an example.

Therefore, we have the following possibilities.

D.1 A nested balanced incomplete-block design with small blocks $(B)$ 
nested in large blocks $(D)$, in which letters $(L)$ satisfy $L \triangleright B, L \triangleright D$ and $L \wedge D=E$, where $n_{D}>n_{L}$ so that $D$ is not balanced with respect to $L$. Here $\mathcal{F}=\{B, D, L\}$.

D.2 A nested balanced incomplete-block design with small blocks $(B)$ nested in large blocks $(D)$, in which letters $(L)$ satisfy $L \triangleright B, L \triangleright D$ and $L \wedge D=E$, where $n_{D}=n_{L}$ so that $L \bowtie D$. Here $\mathcal{F}=\{B, D, L\}$.

D.3 A nested balanced block design with small blocks $(B)$ nested in large blocks $(D)$, in which letters $(L)$ satisfy $L \triangleright B, L \triangleright D, L \wedge D \neq E$ but $L \wedge B=E$, where $n_{D}>n_{L}$ so that $D$ is not balanced with respect to $L$. Here $\mathcal{F}=\{B, D, L\}$.

D.4 A nested balanced block design with small blocks $(B)$ nested in large blocks $(D)$, in which letters $(L)$ satisfy $L \triangleright B, L \triangleright D$ and $L \wedge B \neq E$, where $n_{D}>n_{L}$ so that $D$ is not balanced with respect to $L$. Here $\mathcal{F}=\{B, D, L\}$.

D.5 A nested balanced block design with small blocks $(B)$ nested in large blocks $(D)$, in which letters $(L)$ satisfy $L \triangleright B, L \triangleright D, L \wedge D \neq E$ but $L \wedge B=E$, where $n_{D}=n_{L}$ so that $L \bowtie D$. Here $\mathcal{F}=\{B, D, L\}$.

D.6 A nested balanced block design with small blocks $(B)$ nested in large blocks $(D)$, in which letters $(L)$ satisfy $L \triangleright B, L \triangleright D$ and $L \wedge B \neq E$, where $n_{D}=n_{L}$ so that $L \bowtie D$. Here $\mathcal{F}=\{B, D, L\}$.

D.7 Small blocks $(B)$ are nested in large blocks $(D)$, and letters $(L)$ are arranged so that $B \triangleright L$ and $D \triangleright L$. Also, $n_{L}=n_{B}$, so that $B \bowtie L$. Here $\mathcal{F}=\{B, D, L\}$.

D.8 Small blocks $(B)$ are nested in large blocks $(D)$, and letters $(L)$ are arranged so that $B \triangleright L$ and $D \triangleright L$. Also, $n_{L}>n_{B}$, so that $L$ is not balanced with respect to $B$. Here $\mathcal{F}=\{B, D, L\}$.

D.9 Small blocks $(B)$ are nested in large blocks $(D)$, and letters $(L)$ are arranged so that $D \triangleright L \triangleright B$, where $n_{B}>n_{L}>n_{D}$, so that $B$ is not balanced with respect to $L$, and $L$ is not balanced with respect to $D$. Here $\mathcal{F}=\{B, D, L\}$.

D.10 A row-column design in which letters $(L)$ are arranged so that $L \triangleright R$ and $L \triangleright C, n_{L}<n_{R}$ and $n_{L}<n_{C}$. Here $\mathcal{F}=\{R, C, L\}$.

D.11 A row-column design in which letters $(L)$ are arranged so that $R \triangleright L, C \triangleright L$, and adjusted orthogonality is achieved. Here $\mathcal{F}=\{R, C, L\}$. This is called triple array when $R \wedge C=L \wedge C=$ $L \wedge R=E$. See Section 13 . 


\begin{tabular}{|c|c|c|c|c|c|}
\hline & $A$ & $B$ & $A$ & $B$ & $C$ \\
\hline$A$ & & $C$ & $B$ & $C$ & $A$ \\
\hline$B$ & $C$ & & $C$ & $A$ & $B$ \\
\hline$A$ & $B$ & $C$ & & $A$ & $B$ \\
\hline$C$ & $A$ & $B$ & $A$ & & $C$ \\
\hline$B$ & $C$ & $A$ & $B$ & $C$ & \\
\hline
\end{tabular}

Figure 25: A design for 30 units, with partitions $F, G$ and $H$ into letters, rows and columns, in which $G \bowtie H, F \triangleright G, F \triangleright H$ and $F$ is balanced with respect to $\{G, H\}$ : blank cells denote empty row-column intersections

D.12 A row-column design in which letters $(L)$ are arranged so that $R \triangleright$ $L, C \triangleright L$, and adjusted orthogonality is not achieved, even though $n_{L} \geq n_{R}+n_{C}-1$. Here $\mathcal{F}=\{R, C, L\}$.

D.13 A row-column design in which letters $(L)$ are arranged so that $R \triangleright$ $L, C \triangleright L$, and adjusted orthogonality cannot be achieved, because $n_{L}<n_{R}+n_{C}-1$. Here $\mathcal{F}=\{R, C, L\}$.

D.14 A row-column design in which letters $(L)$ are arranged so that $R \triangleright$ $L \triangleright C$. Here $\mathcal{F}=\{R, C, L\}$.

\subsection{Three partitions: three relations of balance}

Suppose that $\mathcal{F}=\{F, G, H\}$ and all the pairwise relations are nonorthogonal strict balance in at least one direction. Then $\mathcal{F}$ is supreme. There is no loss of generality in assuming that $n_{F} \leq n_{G} \leq n_{H}$.

If $n_{F}=n_{G}=n_{H}$ then $F \bowtie G, F \bowtie H$ and $G \bowtie H$. As Section 9.4 shows, either each partition is balanced with respect to the other pair, or none is.

If $n_{F}=n_{G}<n_{H}$ then it can happen that $F$ and $G$ have adjusted orthogonality with respect to $H$, in which case each is balanced with respect to the other pair, as shown in Section 9.4. Figure 15 shows an example. Such designs were called pergolas in [181], which was presented by Preece at the 1997 BCC at Queen Mary University of London. It may be possible to have such balance without adjusted orthogonality.

If $n_{F}<n_{G}=n_{H}$ then $F$ may or may not be balanced with respect to $\{G, H\}$. Figure 25 shows an example in which $N_{F G} N_{G H} N_{H F}$ is completely symmetric because $N_{G H}$ is completely symmetric and $N_{F G}=N_{F H}$. In fact, the triple $(F, G, H)$ satisfies condition (9.3). However, neither $G$ nor $H$ is balanced with respect to the other pair. 
In a real experiment, it is unlikely that two inherent partitions would have a non-orthogonal relation of strict balance. If partition $L$ is of interest and partition $R$ is not then the desirability of variance balance means that it is more usual to have $L \triangleright R$ than $R \triangleright L$. If $R$ is inherent then there is usually no need for it to be balanced with respect to any subset of other partitions. Thus statisticians have not investigated the other possibilities.

Therefore, we have the following list of possible structures, which is not divided as finely as the lists in previous subsections. In every case, $\mathcal{F}=\{F, G, H\}$.

E.1 $F \bowtie G, F \bowtie H$ and $G \bowtie H$, and each partition is balanced with respect to the other pair. See Section 14 .

E.2 $F \bowtie G, F \bowtie H$ and $G \bowtie H$, but no partition is balanced with respect to the other pair.

E.3 $F \bowtie G, F \triangleright H, G \triangleright H, n_{F}<n_{H}$, and $F$ and $G$ have adjusted orthogonality with respect to $H$, which forces each of $F$ and $G$ to be balanced with respect to the other pair.

E.4 $F \bowtie G, F \triangleright H, G \triangleright H, n_{F}<n_{H}$, and $F$ and $G$ do not have adjusted orthogonality with respect to $H$, but nonetheless each of $F$ and $G$ is balanced with respect to the other pair.

E.5 $F \bowtie G, F \triangleright H, G \triangleright H, n_{F}<n_{H}$, and neither of $F$ and $G$ is balanced with respect to the other pair.

E.6 $F \triangleright G, F \triangleright H, G \bowtie H, n_{F}<n_{H}$ and $F$ is balanced with respect to $\{G, H\}$.

E.7 $F \triangleright G, F \triangleright H, G \bowtie H, n_{F}<n_{H}$ and $F$ is not balanced with respect to $\{G, H\}$.

E.8 $F \triangleright G \triangleright H$ and $n_{F}<n_{G}<n_{H}$.

\section{Youden squares and their generalizations}

\subsection{A rectangle with one set of letters}

Definition An incomplete-block design is symmetric if both partitions are uniform and they have the same number of parts.

This word 'symmetric' is standard, but it is unfortunate, because it does not imply that the incidence matrix is symmetric or that there is an automorphism interchanging the two partitions. The adjective square is also used: see $[45,52]$. 


\begin{tabular}{|c|c|c|c|c|c|c|}
\hline$A$ & $B$ & $C$ & $D$ & $E$ & $F$ & $G$ \\
\hline$B$ & $D$ & $F$ & $E$ & $G$ & $A$ & $C$ \\
\hline$C$ & $F$ & $E$ & $A$ & $B$ & $G$ & $D$ \\
\hline
\end{tabular}

(a)

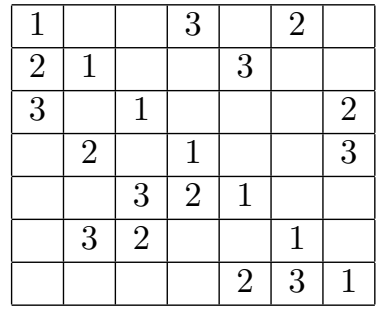

(b)

Figure 26: Different representations of the same $3 \times 7$ Youden square

Definition An $n \times m$ Youden square is an $n \times m$ rectangle in which $R \wedge C=E$ and $R \perp C$, so that there is exactly one cell in each row-column intersection, with one of $m$ letters allocated to each cell, in such a way that (i) each letter occurs once in each row and (ii) the allocation of letters to columns forms a balanced binary incomplete-block design. Thus $n<m$.

These designs were introduced by Youden in [230]. His first example, with $n=3$ and $m=7$, is shown in Figure 26(a). If the roles of letters and columns are interchanged, the design can be represented as an $m \times m$ square with $m(m-n)$ empty cells, as in Figure 26(b). This representation led to the confusing name 'Youden square', which has been used since [80].

Deletion of any row of an $m \times m$ Latin square gives an $(m-1) \times m$ Youden square. Yates had recommended these in [226]. How can they be constructed if $n<m-1$ ?

In [230], Youden wrote that 'some patience is required to obtain' the rectangular layout from the incomplete-block design. In [196], Smith and Hartley proved that this is always possible, whether or not the incompleteblock design is balanced. In fact, this follows from Hall's Marriage Theorem [89], as shown in [28, Chapter 11]. There is a greedy algorithm, where one row is constructed at a time as a 'system of distinct representatives' of the columns; then induction is used. This argument generalizes easily to situations where condition (i) is weakened to 'all letters appear equally often in each row' and/or the words 'binary incomplete' in condition (ii) are weakened to 'generalized binary but not orthogonal', in which case $n$ may be greater than $m$.

Relaxing condition (ii) allows a design such as that in Figure 27, given in [98]. Condition (i) was weakened as above in [4, 192]. Doing both gives what Kiefer called regular generalized Youden designs in [109].

However, when both conditions are weakened to 'strictly balanced but not orthogonal' then the method of constructing transversals by using 


\begin{tabular}{|c|c|c|c|c|c|c|}
\hline$A$ & $C$ & $C$ & $B$ & $D$ & $B$ & $D$ \\
\hline$D$ & $A$ & $B$ & $C$ & $C$ & $D$ & $A$ \\
\hline$B$ & $D$ & $D$ & $A$ & $A$ & $C$ & $B$ \\
\hline$C$ & $B$ & $A$ & $D$ & $B$ & $A$ & $C$ \\
\hline
\end{tabular}

Figure 27: A regular generalized Youden square, relaxing condition (ii), shown with rows and columns interchanged

Hall's Marriage Theorem is no longer guaranteed to work, and so Kiefer had to give many explicit constructions in [109]. Further non-regular generalized Youden designs are in $[15,188]$.

Preece gave many direct constructions of Youden squares, and classified them up to various notions of isomorphism, in [146, 158, 160, 165]. The review in [158] was presented to the 1989 BCC in Norwich.

\subsection{Double Youden rectangles}

If a Youden square is a poor man's Latin square, what should a poor man's Graeco-Latin square be? We already have partitions $R, C$ and $L$ with $n_{R}<n_{C}=n_{L}$. Should the new partition $G$ have $n_{G}=n_{R}$ or $n_{G}=n_{C}$ ? If $n_{G}=n_{C}$ then we have three partitions with pairwise strict balance. In this case, as we saw in Section 9, we need to consider their ternary relation as well, so we defer this to Section 14.3.

Definition An $n \times m$ double Youden rectangle is an $n \times m$ rectangle with partitions $R$ (rows), $C$ (columns), $L$ (Latin letters) and $G$ (Greek letters) such that $n_{R}=n_{G}=n<m, n_{C}=n_{L}=m, R \perp C, R \perp L, G \perp C$, $G \perp L, R \bowtie G$ and $C \bowtie L$, and $R \wedge C=R \wedge L=G \wedge C=G \wedge L=E$. This means that every row meets every column and every Latin letter in exactly one cell, every Greek letter meets every Latin letter and every column in exactly one cell, the relation between Latin letters and columns is a symmetric binary balanced block design, while the relation between Greek letters and rows is a symmetric generalized binary balanced block design.

Thus removal of $G$ leaves a Youden square, while removal of $L$ leaves one of the weak generalizations of Youden squares. This name was given in [22], but the concept was not new. Clarke had given the $4 \times 5$ double Youden rectangle in Figure 28 in [59], and Preece had given the $4 \times 13$ double Youden rectangle in Figure 29 in [156]. Clarke also gave a $5 \times 6$ double Youden rectangle in [59] and a $4 \times 7$ one in [60], while Freeman gave 


\begin{tabular}{|ll|ll|ll|ll|ll|}
\hline$B$ & $\alpha$ & $E$ & $\alpha$ & $D$ & $\delta$ & $A$ & $\beta$ & $C$ & $\gamma$ \\
\hline$C$ & $\beta$ & $A$ & $\gamma$ & $E$ & $\beta$ & $B$ & $\delta$ & $D$ & $\alpha$ \\
\hline$D$ & $\gamma$ & $C$ & $\delta$ & $A$ & $\alpha$ & $E$ & $\gamma$ & $B$ & $\beta$ \\
\hline$E$ & $\delta$ & $D$ & $\beta$ & $B$ & $\gamma$ & $C$ & $\alpha$ & $A$ & $\delta$ \\
\hline
\end{tabular}

Figure 28: A $4 \times 5$ double Youden rectangle

\begin{tabular}{|c|c|c|c|c|c|c|c|c|c|c|c|c|c|}
\hline$A \mathbf{Q}$ & C, & 48 & 78 & 8 ? & ds & 10 & & & & $Q$ & & & \\
\hline $2 \diamond$ & 50 & $3 \diamond$ & $4 \boldsymbol{~}$ & 6 个 & $7 \diamond$ & 84 & 9 क & $10 \stackrel{9}{9}$ & $K \varnothing$ & $J$ & & 28 & \\
\hline 4 ? & $J \diamond$ & 6 < & $K \diamond$ & $5 \diamond$ & 9 C & 7 & 380 & $Q$ & 10 A & $A$ & & 20 & \\
\hline ) & C & C & 5 & $A \varnothing$ & 68 & 3 & 4 & $y$ & & 7 & & & \\
\hline
\end{tabular}

Figure 29: A $4 \times 13$ double Youden rectangle

some designs in [82] which Preece was able to interpret in [150] as double Youden rectangles, including one of size $6 \times 7$. This is useful because this size is excluded from the construction by Hedayat, Parker and Federer in [93], which uses an $(m-1) \times(m-1)$ Graeco-Latin square with a transversal to build an $(m-1) \times m$ double Youden rectangle. Saha and Das gave a construction for size $(m-1) \times m$ when $m$ is odd in [186]. Some of these small double Youden rectangles are also given in [145]. Several of size $5 \times 11$ are in [152].

The review paper [158] seems to have spurred Preece and his collaborators to find further double Youden rectangles. One of size $6 \times 11$ is given in [159]. Preece gave several $7 \times 15$ double Youden rectangles in [160], including the interesting one in Figure 30. Here the Youden square given by ignoring the Greek letters can be obtained from projective geometry using the methods of [75] and [97, Section 17.5]: it admits the alternating group $A_{7}$ as a group of automorphisms. An interesting connection with Kirkman's schoolgirls problem is in [88].

Christofi enumerated $4 \times 5$ and $5 \times 6$ double Youden rectangles in [57], which was presented at the BCC in Surrey (1991), and those of size $6 \times 7$ in [58]. Preece gave some double Youden rectangles of sizes $8 \times 15,5 \times 11$ and $6 \times 11$ in [161], [162] and [165] respectively. The last two were presented at the BCCs in Surrey (1991) and Stirling (1995).

In [214], Vowden gave a construction for $p \times(2 p+1)$ double Youden rectangles for all primes $p$ congruent to 3 modulo 4 , excluding the size $3 \times 7$, for which there is no such rectangle. This was augmented in [172] by the results of a computer search to give double Youden rectangles of 


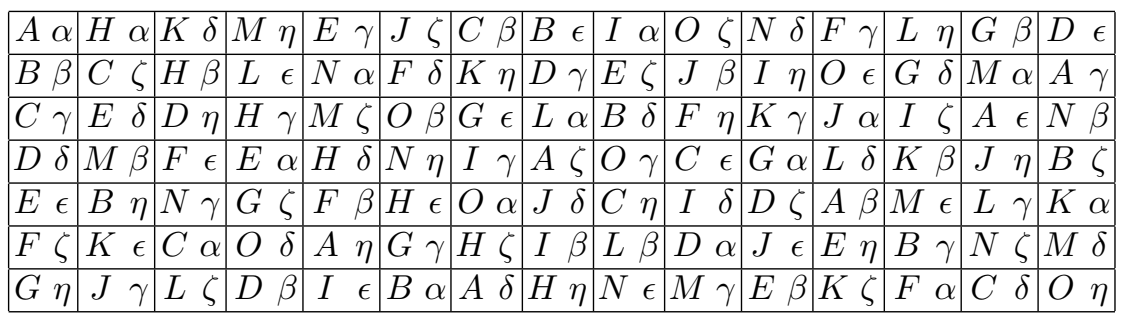

Figure 30: A $7 \times 15$ double Youden rectangle

sizes $p \times(2 p+1)$ and $(p+1) \times(2 p+1)$ for $p$ in $\{5,7,9,11\}$. Vowden adapted his construction to give $p \times(2 p+1)$ double Youden rectangles for prime powers $p$ congruent to 1 modulo 4 , excluding $p=5$, in [215]. Further computer searches, described in [137], gave positive results for sizes $5 \times 21$, $6 \times 31$ and $8 \times 57$ in [173], and size $9 \times 37$ in [138].

\section{Nested balanced incomplete-block designs}

\subsection{Small blocks inside large blocks}

In the original idea for nested BIBDs, there are two inherent uniform parititions $B$ and $D$ of $\Omega$. The parts of $B$ are small blocks, and the parts of $D$ are large blocks, each comprising $k_{D} / k_{B}$ small blocks. Given a set of $n_{L}$ letters, the aim is to allocate letters to experimental units in such a way that $L$ and $B$ form one BIBD and $L$ and $D$ form another.

Definition Uniform partitions $B, D$ and $L$ on a set $\Omega$ form a nested balanced-incomplete block design if $B \prec D$ and $L$ is binary balanced with respect to each of $B$ and $D$ separately but not orthogonal to either.

Preece defined these designs in [148], where he cited two previous uses $[106,111]$ in scientific experiments, and, as was usual for him, gave a table of designs for small sizes, many of them made by cyclic development from one or more initial small blocks (parts of $B$ ) and large blocks (parts of $D$ ).

Constructions based on finite fields were given in [64, 101]. Designs with $n_{D}=n_{L}, k_{D}=n_{L}-1$ and $k_{B}=2$ were given for all odd $n_{L}$ in [36].

Morgan included such designs in his survey chapter [119]. Preece talked about them at the 1999 BCC in Kent, attended by Morgan. As a result, they teamed up, and together with Rees, generalized the concept, first to doubly nested BIBDs, and then to multiply nested BIBDs. The former 
have $B \prec D_{1} \prec D_{2}$ with $L$ binary balanced with respect to each of $B$, $D_{1}$ and $D_{2}$, while the latter is the obvious generalization. Paper [170] is about doubly nested BIBDS, while [121] is a much expanded and updated survey, including the observation that most designs for whist tournaments can be considered as nested BIBDS: see [14].

As discussed in Section 6.2.1, if $B$ has fixed effects then there is no benefit from having $L$ balanced with respect to $D$. Preece was clear in [148] that he was assuming random effects of small blocks. As the ratio $\sigma_{B}^{2} / \sigma^{2}$ increases, there is less need to have $L$ balanced with respect to $D$.

\subsection{Nested row-column designs}

These ideas can be extended to the poset block structure in Example 4.4, with partitions $B, R$ and $C$ into blocks, rows and columns. If $B$, $R$ and $C$ all have fixed effects, then it is desirable to allocate letters in such a way that $L$ is balanced with respect to $\{R, C, B\}$. As shown in Section 9.2 , this is equivalent to $L$ being balanced with respect to $\{R, C\}$.

Some nested row-column designs with this property and with $L$ being binary with respect to $B$ are given in $[8,9,56,124,194,200,203,209$, $210,211,212]$. The lack of specified binary relations between $L$ and $B, L$ and $R$, and $L$ and $C$ takes us somewhat outside the scope of this paper, but there is an excellent survey in [119]. The square in Figure 14 gives an example with $n_{B}=1$.

If $n_{L}<k_{B}$ then it seems intuitive that the best designs should be among those for which $L$ is binary with respect to blocks. However, Bagchi, Mukhopadhyay and Sinha [20] and Chang and Notz [55] showed independently in 1990 that, under fixed effects, a design like the one in Figure 13, in which, in every block, each row has the same set of letters, gives the smallest average variance of the estimators of differences $\tau_{i}-\tau_{j}$.

On the other hand, if rows and columns have random effects with small variances then the arguments in $[24,56]$ show that there is a reason to allocate the parts of $L$ in such a way that $L \triangleright G$ for all $G$ in $\{R, C, B\}$. That is, letters should be allocated so that they form a BIBD in rectangles, in rows and in columns separately. Several constructions for this are given in [148]. One with seven letters in seven $2 \times 3$ rectangles is obtained by developing

\begin{tabular}{|l|l|l|}
\hline 2 & 3 & 5 \\
\hline 0 & 6 & 4 \\
\hline
\end{tabular}

modulo 7. In [122], Morgan and Uddin gave an inequality involving $\sigma^{2}$, $\sigma_{R}^{2}, \sigma_{C}^{2}$ and $\sigma_{B}^{2}$ which determines exactly when such a design is better than one like that in Figure 13. 


\section{Triple arrays}

\subsection{Definitions and examples}

Definition Consider an $n \times m$ rectangle in which one of $v$ letters is allocated to each cell. This is a double array if the partitions $R$ (rows), $C$ (columns) and $L$ (letters) are all uniform with pairwise binary relations such that $R \perp C, R \triangleright L$ and $C \triangleright L$. It is a triple array if it is a double array and, additionally, $R$ and $C$ have adjusted orthogonality with respect to $L$. Thus we have an $n \times m$ rectangle with precisely one unit in each row-column intersection. Letters are allocated to units, in such a way that no letter is repeated in any row or column. It is a double array if it satisfies the two conditions (i) every pair of rows has the same number of letters in common, and (ii) every pair of columns has the same number of letters in common. If, in addition, (iii) every row has $n m / v$ letters in common with every column, then it is a triple array.

Figure 4 shows a triple array with $n=5, m=6$ and $v=10$.

These definitions were given by McSorley, Phillips, Wallis and Yucas in [118], but without mentioning 'adjusted orthogonality'. Instead, they gave condition (8.5), as Preece had done in [145, 147]. They mentioned one design given in [143] and one in [145], and referred to a table of design parameters in [151]. Being unaware of the literature on adjusted orthogonality, they did not realise that Anderson and Eccleston [12] and Bagchi and Shah [21] had also written about such designs, and they proved Theorem 8.5, which Bagchi had published in [18].

Preece became aware of [118] before publication. He began joint research with its authors, adopted the name 'triple arrays' with enthusiasm, and talked about them in the 2003 BCC in Bangor: see [139]. McSorley also talked about related work there.

Triple arrays which meet the bound in Theorem 8.5 are very interesting. They were called extremal in [37], which, being unpublished, had not been seen by McSorley and his co-authors. The following two examples of extremal triple arrays are taken from [37].

Example 13.1 Replacing the letters $A-I$ in Figure 4 lexicographically by the unordered pairs from $\{1,2,3,4,5\}$ gives the design in Figure 31. The rows can be labelled 1-5 and the columns labelled by six pentagons with vertex-set $\{1,2,3,4,5\}$ which form a single orbit under the action of the alternating group $A_{5}$. Then the unordered pair in row $i$ and column $j$ is the edge opposite to vertex $i$ in pentagon $j$.

Thus $A_{5}$ acts as a group of automorphisms of the array in Figure 31 fixing each of the three partitions. In the action on ordered pairs from 


\begin{tabular}{|l|l|l|l|l|l|}
\hline 34 & 45 & 35 & 25 & 24 & 23 \\
\hline 45 & 35 & 34 & 14 & 13 & 15 \\
\hline 15 & 24 & 12 & 45 & 25 & 14 \\
\hline 12 & 13 & 25 & 23 & 15 & 35 \\
\hline 23 & 12 & 14 & 13 & 34 & 24 \\
\hline
\end{tabular}

Figure 31: Relabelled version of Figure 4

\begin{tabular}{|l|c|c|c|c|c|c|c|c|c|}
123 & 124 & 125 & 126 & 134 & 135 & 136 & 145 & 146 & 156 \\
456 & 356 & 346 & 345 & 256 & 246 & 245 & 236 & 235 & 234 \\
\hline 23 & 24 & 25 & 26 & 34 & 35 & 36 & 45 & 46 & 56 \\
\hline 13 & 14 & 15 & 16 & 56 & 46 & 45 & 36 & 35 & 34 \\
\hline 12 & 56 & 46 & 45 & 14 & 15 & 16 & 26 & 25 & 24 \\
\hline 56 & 12 & 36 & 35 & 13 & 26 & 25 & 15 & 16 & 23 \\
\hline 46 & 36 & 12 & 34 & 26 & 13 & 24 & 14 & 23 & 16 \\
\hline 45 & 35 & 34 & 12 & 25 & 24 & 13 & 23 & 14 & 15 \\
\hline
\end{tabular}

Figure 32: An extremal triple array with $n=6, m=10$ and $v=15$

$\{1,2,3,4,5\}$, the irreducible subspaces of this action have dimensions 1,4 and 5 . Because rows and columns are both balanced with respect to letters, $\operatorname{dim}\left(V_{L R}\right)=n-1=4$ and $\operatorname{dim}\left(V_{L C}\right)=m-1=5$. Both subspaces are fixed by the automorphisms, so they must be the two non-trivial irreducible subspaces and so they are orthogonal to each other. The discussion in Section 8.2 shows that this array has adjusted orthogonality. It is an extremal triple array because $n+m-1=10=v$.

Example 13.2 Figure 32 shows an extremal triple array with $n=6$, $m=10$ and $v=15$. The letters are shown as unordered pairs from $\{1,2,3,4,5,6\}$; the rows are labelled $1-6$ in natural order, and the columns are labelled by the partitions of $\{1,2,3,4,5,6\}$ into two parts of size three. The unordered pair in row $i$ and column $j$ consists of the two numbers in the same part as $i$ in partition $j$. The symmetric group $S_{6}$ acts a group of automorphisms of this design, and so a similar argument to that in Example 13.1 shows that this is a triple array.

Sterling and Wormald gave extremal triple arrays of sizes $4 \times 9,5 \times 16$ and $6 \times 25$ in [201].

Example 13.3 The non-extremal triple array shown in Figure 33 was given by McSorley et al. in [118], thereby answering a question posed by 


\begin{tabular}{|c|c|c|c|c|c|c|c|c|c|c|c|c|c|c|}
\hline$e$ & $A$ & $R$ & $P$ & $G$ & $J$ & $E$ & $C$ & $D$ & $B$ & $g$ & $N$ & $S$ & $O$ & $L$ \\
\hline$Z$ & $f$ & $A$ & $B$ & $c$ & $d$ & $b$ & $T$ & $a$ & $K$ & $E$ & $h$ & $C$ & $H$ & $D$ \\
\hline$A$ & $Q$ & $M$ & $I$ & $C$ & $D$ & $U$ & $V$ & $F$ & $i$ & $Y$ & $E$ & $X$ & $B$ & $W$ \\
\hline$F$ & $a$ & $g$ & $b$ & $P$ & $M$ & $i$ & $d$ & $O$ & $J$ & $I$ & $Z$ & $L$ & $Q$ & $c$ \\
\hline$P$ & $L$ & $W$ & $f$ & $h$ & $U$ & $O$ & $g$ & $X$ & $V$ & $K$ & $J$ & $H$ & $Y$ & $T$ \\
\hline$U$ & $V$ & $b$ & $X$ & $Y$ & $S$ & $G$ & $N$ & $R$ & $c$ & $a$ & $W$ & $Z$ & $d$ & $e$ \\
\hline$K$ & $G$ & $H$ & $N$ & $M$ & $f$ & $T$ & $F$ & $h$ & $R$ & $S$ & $Q$ & $i$ & $e$ & $I$ \\
\hline
\end{tabular}

Figure 33: A non-extremal triple array with $n=7, m=15$ and $v=35$

Preece at the 1973 BCC in Aberystwyth and listed in [151] as unresolved. Here $n=7, m=15$ and $v=35$. Phillips and Wallis described in [140] how the design had been found by a computer search. Yucas showed in [231] how it can be obtained from projective 3-space over GF(2), which suggests an intriguing connection with the design in Figure 30.

\subsection{The connection with symmetric BIBDs}

Given an extremal triple array, construct a symmetric incomplete-block design (in the usual combinatorialist's sense) with $v+1$ blocks of size $n$ as follows. The points are $\left\{u_{1}, \ldots, u_{n}, w_{1}, \ldots, w_{m}\right\}$. Considered as a set of points, block $i$ is

$\left\{w_{j}\right.$ : letter $i$ occurs in column $\left.j\right\} \cup\left\{u_{\ell}\right.$ : letter $i$ does not occur in row $\left.\ell\right\}$

for $i=1, \ldots, v$, and block $v+1$ is $\left\{u_{1}, \ldots, u_{n}\right\}$.

The following, proved in [118] and [37], and was implicit in [6].

Theorem 13.4 The symmetric incomplete-block design made from an extremal triple array by the foregoing construction is balanced.

The common concurrence in this block design is $n(v-m) / v$, and so the difference between the block size and this is $n m / v$, which is the number of times that each letter occurs in the triple array, which I have been writing as $k_{L}$. As proved in [116], this is also the number of letters which any row and column have in common.

Can we reverse the foregoing construction, and make the extremal triple array from the symmetric BIBD? Here is another piece of interesting history. In 1966, Agrawal published several papers in this area. Paper [6] is particularly relevant: it constructs some of the same designs that Preece was constructing, and it gives condition (8.5). It is not surprising 
that Preece and Agrawal were unaware of each other's work published in the same year, but Preece became aware of Agrawal's work and cited it in [151].

Given the symmetric BIBD, we label the rows of the $n \times m$ rectangle by the points in the first block and label the columns by the other points. Then ignore that block. For row $i$, write down the set of other blocks that do not contain point $i$; for column $j$, write down the set of other blocks that do contain point $j$. Put the block-names in column $j$ in any order: then it is simply a matter of re-arranging the names in each column in such a way that each row has the correct set of names.

'Simply'? This runs into exactly the same problem that we met for nonregular generalized Youden designs in Section 11.1: the method of selecting a system of distinct representatives used in Hall's Marriage Theorem is no longer guaranteed to work. In fact, when $n=3, m=4$ and $v=6$ (so that $k_{L}=2$ ) there is a symmetric BIBD but no triple array. This leads to the following conjecture in [118].

Conjecture 13.5 If $k_{L}>2$ then the foregoing construction can be reversed to give an extremal triple array from the symmetric BIBD.

Agrawal wrote in [6] that 'In the examples tried by the author it was found that if $k_{L}>2$ such arrangement is always possible (we have no mathematical proof for the above observation).' (His notation has been changed to match that used here.) In [125], Nageswara Rao says that the fact that this 'arrangement of symbols is always possible can be shown with the help of generalized systems of distinct representatives', yet does not cite the earlier paper [178], of which he was apparently a co-author, which claims to give a proof, not excluding the case $k_{L}=2$. The procedure given in [178] finds a set of distinct representatives for each row, with no check to ensure that there are no repeats in any column. Moreover, the final paragraph gives a different short proof (with acknowledgement to the referee) which gives a design which does not have adjusted orthogonality. Wallis and Yucas pointed out flaws in this proof in [218].

Meanwhile, Anderson and Eccleston claimed in [12] that algorithms implemented in software described in [104] and [185] always succeed in creating the row-column design. More detail about the first is in [103].

Here is a way of thinking about the problem. We know the set $\mathcal{R}_{i}$ of $m$ letters that should go in row $i$, and we know the set $\mathcal{C}_{j}$ of $n$ letters that should go in column $j$. Write the set $\mathcal{R}_{i} \cap \mathcal{C}_{j}$ in cell $(i, j)$ to give an array like that in Figure 34. Can we choose just one letter in each cell (an array of distinct representatives) in such a way that the chosen letters in each 


\begin{tabular}{|lll|lll|lll|lll|lll|lll|}
\hline$E$ & $H$ & $J$ & $F$ & $I$ & $J$ & $G$ & $H$ & $I$ & $E$ & $G$ & $J$ & $F$ & $G$ & $H$ & $E$ & $F$ & $I$ \\
\hline$D$ & $H$ & $J$ & $B$ & $I$ & $J$ & $C$ & $H$ & $I$ & $B$ & $C$ & $J$ & $B$ & $D$ & $H$ & $C$ & $D$ & $I$ \\
\hline$A$ & $D$ & $J$ & $A$ & $F$ & $J$ & $A$ & $C$ & $G$ & $C$ & $G$ & $J$ & $D$ & $F$ & $G$ & $C$ & $D$ & $F$ \\
\hline$A$ & $D$ & $E$ & $A$ & $B$ & $I$ & $A$ & $G$ & $I$ & $B$ & $E$ & $G$ & $B$ & $D$ & $G$ & $D$ & $E$ & $I$ \\
\hline$A$ & $E$ & $H$ & $A$ & $B$ & $F$ & $A$ & $C$ & $H$ & $B$ & $C$ & $E$ & $B$ & $F$ & $H$ & $C$ & $E$ & $F$ \\
\hline
\end{tabular}

Figure 34: Array of subsets to illustrate the problem of choosing distinct representatives

row are distinct (and hence make up $\mathcal{R}_{i}$ ) and similarly for columns? In this particular case, the design in Figure 4 gives a solution.

Fon-Der-Flaass considered a more general version of this problem. Given an $n \times m$ array, with a set $\mathcal{S}_{i j}$ of letters in cell $(i, j)$, can we choose one letter from each cell in such a way that the chosen letters in each row are all distinct, as are the chosen letters in each column? In [81], he showed that this decision problem is NP-complete.

\subsection{Paley triple arrays}

If $q$ is an odd prime power then there is a Hadamard matrix of order $2(q+1)$. Any Hadamard matrix can be normalized so that one row has all of its entries equal to +1 . If one column is also normalized, and then that row and column are removed, the positions of the +1 entries in the remaining $(2 q+1) \times(2 q+1)$ matrix form the incidence matrix of a symmetric BIBD for $2 q+1$ points in blocks of size $q$. These designs are sometimes called Hadamard 2-designs: see [52, 96].

If the construction in Section 13.2 can be reversed, this design gives an extremal $q \times(q+1)$ triple array. The triple arrays in Figures 4 and 31 are like this, as are many in $[6,143,145,151]$. Preece pointed out in [151] that a method given by Agrawal and Mishra in [7] could be used to give an extremal $11 \times 12$ triple array.

After Preece's Adelaide talk in 1975, Seberry was motivated to find such arrays. Her paper [187] originally showed Preece as a co-author, but he was so furious at her submitting it without consulting him that he wrote to the journal editor and asked for his name to be removed. ${ }^{2}$ That is why the paper starts with Preece's explanation of the problem, including condition (8.5), followed by the sentence 'We thank D. A. Preece for writing this introduction and now proceed to give our construction and

${ }^{2}$ Personal communication from JS. 
some examples.' The remainder of the paper gives constructions which purport to prove the existence of an extremal $q \times(q+1)$ triple array whenever $q$ is an odd prime power.

In [203], Street also proved this for odd prime powers $q$ with $q \equiv 3$ $(\bmod 4)$, constructing two series of designs not isomorphic to those given in [187]. It was independently proved again for prime powers $q \equiv 3(\bmod 4)$ in [37], this time explicitly excluding $q=3$, for which it is not true.

In [18], Bagchi proved the result for odd prime powers $q$ strictly greater than 3 , unless $q \equiv 1(\bmod 8)$.

Preece, Wallis and Yucas named these Paley triple arrays in [174], and proved the following.

Theorem 13.6 If $q$ is an odd prime power and $q>3$, then there exists an extremal $q \times(q+1)$ triple array.

This is a very nice result, with explicit constructions given and verified. However, the paper does not mention the results in [18] or [203], which independently prove this for some congruence classes modulo 8. It does cite [37, 187], but neither states their results nor finds fault with their proofs. It does credit [10] with giving some 'partial results'. Recently Nilson showed in [129] that some of the constructions in [187] do not give adjusted orthogonality, and Cameron ${ }^{3}$ has independently found similar problems in [187].

\subsection{Recent work}

In [131], Nilson and Heidtmann followed up on the connection between extremal triple arrays and symmetric BIBDs. They defined a property of symmetric BIBDs called inner balance, which is achieved by the symmetric BIBDs with 11 points in blocks of either size five or size six. The ensuing theory enables them to prove that there are no extremal triple arrays for the parameter sets left as undecided in [118]. They gave an infinite family of parameter sets for potential symmetric BIBDs with inner balance. Broughton [48] proved that there are no other possiblities.

Nilson and Öhman show in [132] how some Youden squares may be used to construct extremal triple arrays. In particular, all Paley triple arrays may be constructed like this. They also give a construction using difference sets. Further constructions from difference sets are in [130].

The recent survey paper [217] largely consists of material copied verbatim from $[118,174]$. It mentions none of the literature on adjusted orthogonality.

\footnotetext{
${ }^{3}$ Personal communication from PJC.
} 


\section{Universal balance}

\subsection{Definitions and results}

This section picks up the ideas of Section 9, while specializing them to insist that every partition in the set is balanced with respect to every subset of the others. It introduces the term 'universal balance' as well as Theorem 14.2 and Conjecture 14.4, in an attempt to pull together some different pieces of work into a single framework.

Recall that if $\mathcal{L}$ is a set of partitions of $\Omega$ then $V_{\mathcal{L}}=\sum_{L \in \mathcal{L}} V_{L}$ and $P_{\mathcal{L}}$ denotes the matrix of orthogonal projection onto $V_{\mathcal{L}}$. Moreover, partition $F$ is balanced with respect to $\mathcal{L}$ if $X_{F}^{\top}\left(I_{e}-P_{\mathcal{L}}\right) X_{F}$ is completely symmetric but not zero. The insistence on 'not zero' ensures that $\operatorname{dim}\left(V_{\mathcal{L} \cup\{F\}}\right)=$ $\operatorname{dim}\left(V_{\mathcal{L}}\right)+n_{F}-1$.

Definition Let $\mathcal{F}$ be a set of partitions of $\Omega$. Then $\mathcal{F}$ has universal balance if all pairwise relations between distinct partitions in $\mathcal{F}$ are generalized binary and, whenever $F \in \mathcal{F}$ and $\mathcal{L} \subseteq \mathcal{F} \backslash\{F\}$, then $F$ is balanced with respect to $\mathcal{L}$ but $V_{F}$ is not geometrically orthogonal to $V_{\mathcal{L}}$.

Taking $\mathcal{L}$ to be $\emptyset$ in the above definition shows that if $\mathcal{F}$ has universal balance and $F \in \mathcal{F}$ then $F$ is uniform. Likewise, if $F \in \mathcal{F}, G \in \mathcal{F}$ and $F \neq G$ then $F \bowtie G$ and so $n_{F}=n_{G}$. Write $m$ for the common value of $n_{F}$ for $F$ in $\mathcal{F}$.

These remarks give an inductive proof of the following result, which is stated in [13].

Theorem 14.1 If $\mathcal{F}$ has universal balance, $|\mathcal{F}|=s$, and every partition in $\mathcal{F}$ has $m$ parts of size $k$, then $1+s(m-1) \leq e=m k$.

Write $Q_{\mathcal{L}}$ for $P_{\mathcal{L}}-P_{U}$, simplifying $Q_{\{R\}}$ to $Q_{R}$ and $Q_{\{R, C\}}$ to $Q_{R C}$ as in Section 9. Then the arguments used in Section 8.1 show that the following are equivalent:

(i) $F$ is balanced with respect to $\mathcal{L}$ but $V_{F}$ is not geometrically orthogonal to $V_{\mathcal{L}}$;

(ii) there is some scalar $\mu$ in $(0,1)$ such that $Q_{F} Q_{\mathcal{L}} Q_{F}=\mu Q_{F}$.

When condition (14.1) is satisfied, the proof underlying Theorem 7.1(v) shows that

$$
Q_{\mathcal{L} \cup\{F\}}=Q_{\mathcal{L}}+(1-\mu)^{-1}\left(Q_{F}-Q_{\mathcal{L}} Q_{F}-Q_{F} Q_{\mathcal{L}}+Q_{\mathcal{L}} Q_{F} Q_{\mathcal{L}}\right) .
$$


When $\mathcal{L}=\{G\}$ and $F$ and $G$ are partitions into $m$ parts of size $k$ whose pairwise relation is generalized binary then the value of $\mu$ in condition (14.1) is determined by the values of $m$ and $k$. Writing this common value as $\mu_{1}$, expression (7.2) shows that

$$
\left(1-\mu_{1}\right) Q_{R C}=Q_{R}+Q_{C}-Q_{R} Q_{C}-Q_{C} Q_{R}
$$

whenever $\mathcal{F}$ has universal balance and $R$ and $C$ are distinct partitions in $\mathcal{F}$.

Let $L$ be a third such partition. Then there is a scalar $\mu_{2}$ in $(0,1)$ such that

$$
\left(1-\mu_{1}\right)^{-1} Q_{L}\left(Q_{R}+Q_{C}-Q_{R} Q_{C}-Q_{C} Q_{R}\right) Q_{L}=\mu_{2} Q_{L},
$$

which implies that

$$
2 \mu_{1} Q_{L}-Q_{L} Q_{R} Q_{C} Q_{L}-Q_{L} Q_{C} Q_{R} Q_{L}=\mu_{2}\left(1-\mu_{1}\right) Q_{L}
$$

and hence that

$$
Q_{L} Q_{R} Q_{C} Q_{L}+Q_{L} Q_{C} Q_{R} Q_{L}=\left(2 \mu_{1}-\mu_{2}+\mu_{1} \mu_{2}\right) Q_{L}
$$

Pre- and post-multiplication of equation (14.4) by $X_{L}^{\top}$ and $X_{L}$ respectively gives condition (9.2); pre- and post-multiplication by $Q_{R}$ or $Q_{C}$ prove a stronger version of Proposition 9.3(ii) which includes the fact that the value of $\mu_{2}$ does not depend on which one of $\{R, C, L\}$ is distinguished.

However, when $|\mathcal{L} \cup\{F\}| \geq 4$ then the value of $\mu$ in condition (14.1) can be different for different choices of the distinguished partition. Examples of this were given in $[11,63,145,157]$.

After some manipulation, expression (7.2) and equations (14.1), (14.2), (14.3) and (14.4) show that if $\{R, C, L\}$ has universal balance then

$$
\begin{gathered}
\left(1-\mu_{1}\right)\left(1-\mu_{2}\right) Q_{R C L}=\left(1-\mu_{1}\right)\left(Q_{R}+Q_{C}+Q_{L}\right) \\
-\left(Q_{R} Q_{C}+Q_{C} Q_{R}+Q_{R} Q_{L}+Q_{L} Q_{R}+Q_{C} Q_{L}+Q_{L} Q_{C}\right) \\
+\left(Q_{R} Q_{C} Q_{L}+Q_{L} Q_{C} Q_{R}+Q_{C} Q_{L} Q_{R}+Q_{R} Q_{L} Q_{C}\right. \\
\left.+Q_{L} Q_{R} Q_{C}+Q_{C} Q_{R} Q_{L}\right) .
\end{gathered}
$$

Hence induction gives the following.

Theorem 14.2 If $\mathcal{F}$ has universal balance then $Q_{\mathcal{F}}$ is a linear combination of products of the matrices $Q_{F}$ for $F$ in $\mathcal{F}$. 
Corollary 14.3 If $\mathcal{F}$ has universal balance, $\mathcal{L} \subset \mathcal{F}$ and $F \in \mathcal{F} \backslash \mathcal{L}$ then $X_{F}^{\top} Q_{\mathcal{L}} X_{F}$ is a sum of matrices of the form

$$
N_{F L_{1}} N_{L_{1} L_{2}} \cdots N_{L_{r} F}
$$

where $\left(L_{1}, L_{2}, \ldots, L_{r}\right)$ is a sequence of partitions in $\mathcal{L}$, possibly having repeated entries.

Conjecture 14.4 If $\mathcal{F}$ has universal balance then $Q_{\mathcal{F}}$ is a linear combination of matrices of the form

$$
Q_{F_{1}} Q_{F_{2}} \cdots Q_{F_{r}}+Q_{F_{r}} \cdots Q_{F_{2}} Q_{F_{1}}
$$

for sequences $\left(F_{1}, F_{2}, \ldots, F_{r}\right)$, with no repeated entries, of partitions in $\mathcal{F}$.

Apart from the 'non-zero' check, this would imply that $\mathcal{F}$ has universal balance if, whenever $\mathcal{L} \subset \mathcal{F}$ and $F \in \mathcal{F} \backslash \mathcal{L}$ and $\left(L_{1}, L_{2}, \ldots, L_{r}\right)$ is a sequence of distinct partitions in $\mathcal{L}$, then the matrix

$$
N_{F L_{1}} N_{L_{1} L_{2}} \cdots N_{L_{r} F}+N_{F L_{r}} \cdots N_{L_{2} L_{1}} N_{L_{1} F}
$$

is completely symmetric.

\subsection{Sets of partitions with universal balance}

Assume that $s \geq 3$, so that condition (9.2) must be satisfied for all sets of three partitions in $\mathcal{F}$. We know only three families of incidence matrices which satisfy this and the more general condition given at the end of Section 14.1. Those in Section 14.2.1 have every pairwise incidence matrix equal to $J_{m m}-I_{m}$, and there is a direct construction of the partitions on the set $\Omega$. For those in Section 14.2.2, each pairwise incidence matrix is one of only two possibilities, which are the transposes of each other and commute with each other. The pairwise incidence matrices of those in Section 14.2.3 satisfy the stronger condition (9.3). For these last two, we defer the construction of the designs until Section 14.3.

14.2.1 Constructions from orthogonal Latin squares Probably the earliest example of universal balance to be identified has $n_{F}=m=k_{F}+1$ for all $F$ in $\mathcal{F}$. The starting point is a set of $s-2$ mutually orthogonal $m \times m$ Latin squares with a common transversal, where $s \geq 2$. Use the common transversal to label the rows, columns and letters of each square. Then $\Omega$ consists of the $m(m-1)$ cells not in that transversal, and $\mathcal{F}=\left\{R, C, L_{1}, \ldots, L_{s-2}\right\}$, where the parts of $R, C$ and $L_{i}$ are rows, columns and the letters of the $i$-th square. The common labelling ensures that all of the incidence matrices $N_{F G}$ for $F \neq G$ with $\{F, G\} \subseteq \mathcal{F}$ satisfy $N_{F G}=J_{m m}-I_{m}$. See $[5,13,94,176,193]$. 
14.2.2 Constructions from doubly regular tournaments Many of the designs in [11, 145, 147, 203] belong to another infinite family. The design in Figure 16(b) gives an example. Here $N_{R C}=I_{7}+A$ and $N_{C L}=N_{L R}=$ $I_{7}+A^{\top}$, where $A(i, j)=1$ if $j-i$ is a non-zero square in $\operatorname{GF}(7)$ and $A(i, j)=0$ otherwise. These matrices satisfy condition (9.2) but not the stronger condition (9.3), or the intermediate one that $N_{L R} N_{R C} N_{C L}$ is completely symmetric.

Given a directed graph with $m$ vertices, its adjacency matrix $A$ is the $m \times m$ matrix with $A(i, j)=1$ if there is an edge from vertex $i$ to vertex $j$ and $A(i, j)=0$ otherwise. The directed graph is called a doubly regular tournament if $A+A^{\top}=J_{m m}-I_{m}$ and

$$
A A^{\top}=\frac{m+1}{4} I_{m}+\frac{m-3}{4} J_{m m}
$$

from which it follows that

$$
A^{2}=\frac{m+1}{4}\left(J_{m m}-I_{m}\right)-A
$$

and $m \equiv 3(\bmod 4)$. See $[182]$ for more details.

Many doubly regular tournaments come from finite fields. If $m$ is power of a prime and $m \equiv 3(\bmod 4)$ then make a directed graph whose vertices are the elements of $\operatorname{GF}(m)$ with an edge from $i$ to $j$ if $j-i$ is a non-zero square.

Suppose that $e=m k$ and every partition in $\mathcal{F}$ has $m$ parts of size $k$. If there is a doubly regular tournament of size $m$ and the parts of every partition in $\mathcal{F}$ can be labelled by its vertices in such a way that either (i) $k=(m+1) / 2$ and every $m \times m$ incidence matrix is equal to either $I+A$ or $I+A^{\top}$ or (ii) $k=(m-1) / 2$ and every $m \times m$ incidence matrix is equal to either $A$ or $A^{\top}$, then equations (14.5) and (14.6) show that if $M$ is any product of a sequence of such incidence matrices then $M+M^{\top}$ is completely symmetric. Thus Theorem 14.2 shows that $\mathcal{F}$ has universal balance unless there is some $F$ in $\mathcal{F}$ and subset $\mathcal{L}$ of $\mathcal{F} \backslash\{F\}$ such that $X_{F}^{\top}\left(I_{e}-P_{\mathcal{L}}\right) X_{F}$ is zero.

Unlike the straightforward situation in Section 14.2.1, the scalar $\mu$ in condition (14.1) is not determined by the values of $m$ and $k$ when $s \geq 3$. Draw a directed graph with $s$ vertices, one for each partition, and an edge from $F$ to $G$ if $N_{F G}$ includes $A$ rather than $A^{\top}$. The value of $\mu$ depends on the isomorphism class of this directed graph. When $s=3$ it may be a cycle or a total order. There are more possibilities as $s$ increases. See $[3,5,13,40,63,145,169]$. 
14.2.3 Constructions for powers of four As noted in Section 1, many combinatorialists think of block designs as incidence relations between different sets. Working in this mode, Cameron and Seidel developed systems of linked symmetric designs in [50, 53], using permutation groups, Steiner systems and quadratic forms. The pairwise incidence matrices satisfy condition (9.3), and so Theorem 14.2 shows that if these are the incidence matrices of $\mathcal{F}$ then $\mathcal{F}$ has universal balance.

Here is one way of producing the incidence matrices. Start with the Steiner system $\mathfrak{S}(5,8,24)$ : see $[52,114]$. Let $\mathcal{Q}$ be an octad in this, and let $\mathcal{P}$ be the remaining set of 16 points. If $u, w$ are distinct points in $\mathcal{Q}$ then there are precisely 16 octads whose intersection with $\mathcal{Q}$ is $\{u, w\}$ : their intersections with $\mathcal{P}$ give the blocks of a symmetric BIBD with 16 blocks of size six. Call this set of blocks $\mathcal{Q}_{u w}$, and denote the incidence matrix between $\mathcal{P}$ and $\mathcal{Q}_{u w}$ as $N_{0, u w}$.

Let $x$ be a point in $\mathcal{Q} \backslash\{u, w\}$. Then each block of $\mathcal{Q}_{u x}$ intersects six blocks in $\mathcal{Q}_{u w}$ in one point, the remainder in three points. Define a block in $\mathcal{Q}_{u w}$ to be incident with one in $\mathcal{Q}_{u x}$ if they intersect in one point. Then

$$
N_{u w, u x} \text { is a linear combination of } N_{u w, 0} N_{0, u x} \text { and } J_{16,16} \text {. }
$$

Hence $N_{u w, u x} N_{u x, u w}$ is a linear combination of $N_{u w, 0} N_{0, u x} N_{u x, 0} N_{0, u w}$ and $J_{16,16}$, which is completely symmetric because both $N_{0, u x} N_{u x, 0}$ and $N_{u w, 0} N_{0, u w}$ are. Therefore the relation between $\mathcal{Q}_{u x}$ and $\mathcal{Q}_{u w}$ is another symmetric BIBD with 16 blocks of size six.

Moreover, equation (14.7) shows that the triple $\mathcal{P}, \mathcal{Q}_{u x}, \mathcal{Q}_{u w}$ satisfies the strong condition (9.3), as does the triple $\mathcal{Q}_{u x}, \mathcal{Q}_{u w}, \mathcal{Q}_{x w}$ and any triple of the form $\mathcal{Q}_{u x}, \mathcal{Q}_{u w}, \mathcal{Q}_{u v}$ with $v$ in $\mathcal{Q} \backslash\{u, w, x\}$. Thus the relations among $\left\{\mathcal{P}, \mathcal{Q}_{u x}, \mathcal{Q}_{u w}, \mathcal{Q}_{x w}\right\}$ have universal balance, as have those among $\mathcal{P}$ and any collection of $\mathcal{Q}_{u z}$ for $z$ in $\mathcal{Q} \backslash\{u\}$.

Can any of these systems of linked symmetric designs be realised as partitions of a single set of size 96 ? Theorem 14.1 shows that $s \leq 6$, so the whole of the second one cannot be realised without linear dependence. For $s=2$, the pair of partitions simply gives a BIBD. Here we show how to deal with the case $s=3$, and defer the more general case until Section 14.3.

If $\Phi$ is a block of $\mathcal{Q}_{u w}$ then there are six blocks of $\mathcal{Q}_{u x}$ which intersect it in a single point; conversely each point in $\Phi$ occurs in exactly one such intersection. Hence the set of triples $(p, \Phi, \Psi)$ for which $p \in \mathcal{P}, \Phi$ is a block of $\mathcal{Q}_{u w}, \Psi$ is a block of $\mathcal{Q}_{u x}$ and $\Phi \cap \Psi=\{p\}$ has size 96 and admits $\left\{\mathcal{P}, \mathcal{Q}_{u x}, \mathcal{Q}_{u w}\right\}$ as a set of partitions with universal balance.

This construction for $m=16$ is rather special and does not generalize. However, this family of incidence matrices can also be constructed by using quadratic forms, and this approach does generalize to higher powers of 4 : see Section 14.3. 
It is interesting to note that the approach of Cameron and Seidel was based on the idea of equal angles between subspaces, very much as in Section 7. In fact, the definition of universal balance at the start of this section can be interpreted as saying that every vector in $V_{F}$ makes the same angle with $V_{\mathcal{L}}$ (and this angle is neither zero nor $\pi / 2$ ). There is more about the link with equal angles in [49], while [67] shows the interest of this topic to workers in quantum information.

\subsection{Multi-stage Youden rectangles}

Double Youden rectangles provide one generalization of Youden squares. A different generalization, given in $[2,10,13,40,51,54,144,145,147,151$, $169,171,195,216]$, has a set $\mathcal{F}$ of partitions with universal balance, and another partition $G$ orthogonal to all of these. Vowden presented [171] at the Keele BCC in 1993 and [216] at the Stirling BCC in 1995, but the authors later admitted ${ }^{4}$ that the second paper has errors. Many different names have been used: multi-stage or multi-letter, combined with Youden designs or squares or rectangles, and even Freeman-Youden rectangles. Here I choose a name that allows me to present this subsection and the next in a unified way.

Definition Let $n<m$. A multi-stage $n \times m$ Youden rectangle consists of a set $\mathcal{F}$ of at least two partitions of a set $\Omega$ of size $n m$ into $m$ parts of size $n$, such that $\mathcal{F}$ has universal balance, together with a partition $G$ of $\Omega$ into $n$ parts of size $m$ such that $F \wedge G=E$ and $F \perp G$ for all $F$ in $\mathcal{F}$.

If $s=2$ then this is just a Youden square. Most other examples in the literature have $s=3$.

Proposition 14.5 If $n<m$ and there exists a multi-stage $n \times m$ Youden rectangle with $s$ partitions into $m$ parts then $s \leq n$.

The proof is similar to the proof of Theorem 14.1.

For the case that $n=m-1$, if there exists a set of $s-1$ mutually orthogonal $m \times m$ Latin squares then use $s-2$ of these in the construction in Section 14.2.1, taking the common transversal to be the positions of one letter in the remaining Latin square. Then the $n$ other letters of that Latin square give the parts of a partition $G$ orthogonal to all the other partitions.

For most other known sets of partitions with universal balance, the irony is that the simplest way to construct them is to make a multi-stage Youden rectangle and then ignore the distinguished partition $G$.

\footnotetext{
${ }^{4}$ See Mathematical Reviews 1675100
} 


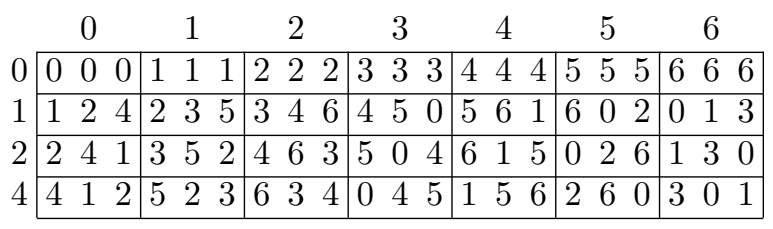

Figure 35: A multi-stage $4 \times 7$ Youden rectangle with four stages: the columns give one stage, the first number in each cell gives the second stage, and the other two numbers in each cell give the remaining two stages

Here is a construction for $n \times m$ multi-stage Youden rectangles when $m$ is an odd prime power $q, q \equiv 3(\bmod 4)$ and $n$ is either $(q-1) / 2$ or $(q+1) / 2$. It uses the doubly regular tournament derived from the set $\mathcal{S}$ of non-zero squares in $\operatorname{GF}(q)$. For $n=(q+1) / 2$, label the rows of a rectangle by the elements of $\{0\} \cup \mathcal{S}$, and label the columns by the elements of $\mathrm{GF}(q)$. The rows form the parts of $G$. For $u \in\{0\} \cup \mathcal{S}$, define the partition $F_{u}$ to be the kernel of the function mapping cell $(i, j)$ to $u i+j$. Thus $F_{0}$ is just the partition into columns. If we use all $(q+1) / 2$ such partitions, the bound in Proposition 14.5 is achieved.

For $n=(q-1) / 2$, remove row 0 from the previous construction. To avoid linear dependence, at most $(q-1) / 2$ of the partitions $F_{u}$ can be used.

Many constructions of multi-stage Youden rectangles given in the literature use a version of the above method. Figure 35 shows the result when $m=q=7$ and $n=4=(q+1) / 2$.

What about the constructions for powers of 4 ? After Preece's talk to the 1973 BCC in Aberystwyth, Cameron told him about the results in Section 14.2.3, and they pooled their ideas to produce the designs with $m=$ 16 and $k=6$ or 10 in [167]. These include the $6 \times 16$ design in Figure 36, where the columns and the letters in each of the three positions in each cell give four partitions with the incidence structure of $\left\{\mathcal{P}, \mathcal{Q}_{u x}, \mathcal{Q}_{u w}, \mathcal{Q}_{x w}\right\}$. The rows form another partition orthogonal to all of these.

It turns out that the Cameron-Seidel construction also has a link with coding theory. A set like $\left\{\mathcal{P}, \mathcal{Q}_{u x}, \mathcal{Q}_{u w}, \mathcal{Q}_{x w}\right\}$ gives a linear code and can be realised as a set of partitions. One like $\left\{\mathcal{P}, \mathcal{Q}_{u x}, \mathcal{Q}_{u w}, \mathcal{Q}_{u v}, \ldots\right\}$ gives a non-linear code (a Kerdock code) and cannot.

In spite of being a co-author of [167], Cameron was still not comfortable thinking in terms of different partitions of a single set. When he finally adjusted to this mindset, he generalized 16 to arbitrary powers $4^{t}$ of 4 , with blocks of size $2^{2 t-1}-2^{t-1}$ or $2^{2 t-1}+2^{t-1}$, and produced the designs in both forms - as a set of incidence relations satisfying (9.3) and as multi- 


\begin{tabular}{|c|c|c|c|c|c|c|c|c|c|c|c|c|c|c|c|}
\hline$A$ & $B$ & $C$ & $D$ & $E$ & $F$ & $G$ & $H$ & $I$ & $J$ & $K$ & $L$ & $M$ & $N$ & $O$ & $P$ \\
$A$ & $B$ & $C$ & $D$ & $E$ & $F$ & $G$ & $H$ & $I$ & $J$ & $K$ & $L$ & $M$ & $N$ & $O$ & $P$ \\
$A$ & $B$ & $C$ & $D$ & $E$ & $F$ & $G$ & $H$ & $I$ & $J$ & $K$ & $L$ & $M$ & $N$ & $O$ & $P$ \\
\hline$H$ & $G$ & $F$ & $E$ & $D$ & $C$ & $B$ & $A$ & $P$ & $O$ & $N$ & $M$ & $L$ & $K$ & $J$ & $I$ \\
$G$ & $H$ & $E$ & $F$ & $C$ & $D$ & $A$ & $B$ & $O$ & $P$ & $M$ & $N$ & $K$ & $L$ & $I$ & $J$ \\
$B$ & $A$ & $D$ & $C$ & $F$ & $E$ & $H$ & $G$ & $J$ & $I$ & $L$ & $K$ & $N$ & $M$ & $P$ & $O$ \\
\hline$K$ & $L$ & $I$ & $J$ & $O$ & $P$ & $M$ & $N$ & $C$ & $D$ & $A$ & $B$ & $G$ & $H$ & $E$ & $F$ \\
$J$ & $I$ & $L$ & $K$ & $N$ & $M$ & $P$ & $O$ & $B$ & $A$ & $D$ & $C$ & $F$ & $E$ & $H$ & $G$ \\
$D$ & $C$ & $B$ & $A$ & $H$ & $G$ & $F$ & $E$ & $L$ & $K$ & $J$ & $I$ & $P$ & $O$ & $N$ & $M$ \\
\hline$M$ & $N$ & $O$ & $P$ & $I$ & $J$ & $K$ & $L$ & $E$ & $F$ & $G$ & $H$ & $A$ & $B$ & $C$ & $D$ \\
$I$ & $J$ & $K$ & $L$ & $M$ & $N$ & $O$ & $P$ & $A$ & $B$ & $C$ & $D$ & $E$ & $F$ & $G$ & $H$ \\
$E$ & $F$ & $G$ & $H$ & $A$ & $B$ & $C$ & $D$ & $M$ & $N$ & $O$ & $P$ & $I$ & $J$ & $K$ & $L$ \\
\hline$O$ & $P$ & $M$ & $N$ & $K$ & $L$ & $I$ & $J$ & $G$ & $H$ & $E$ & $F$ & $C$ & $D$ & $A$ & $B$ \\
$F$ & $E$ & $H$ & $G$ & $B$ & $A$ & $D$ & $C$ & $N$ & $M$ & $P$ & $O$ & $J$ & $I$ & $L$ & $K$ \\
$L$ & $K$ & $J$ & $I$ & $P$ & $O$ & $N$ & $M$ & $D$ & $C$ & $B$ & $A$ & $H$ & $G$ & $F$ & $E$ \\
\hline$P$ & $O$ & $N$ & $M$ & $L$ & $K$ & $J$ & $I$ & $H$ & $G$ & $F$ & $E$ & $D$ & $C$ & $B$ & $A$ \\
$C$ & $D$ & $A$ & $B$ & $G$ & $H$ & $E$ & $F$ & $K$ & $L$ & $I$ & $J$ & $O$ & $P$ & $M$ & $N$ \\
$N$ & $M$ & $P$ & $O$ & $J$ & $I$ & $L$ & $K$ & $F$ & $E$ & $H$ & $G$ & $B$ & $A$ & $D$ & $C$ \\
\hline
\end{tabular}

Figure 36: A multi-stage $6 \times 16$ Youden rectangle with four stages: the columns give one stage, the first letter in each cell gives the second stage, the second and third letters give the other two stages 
stage Youden rectangles. He spoke about these results at the 2001 BCC in Sussex: see [51].

There is also a link with another nice combinatorial object. The complete graph $K_{16}$ on 16 vertices can be decomposed into three strongly regular graphs with valency five. Their edge-sets are disjoint, so their adjacency matrices $A_{1}, A_{2}$ and $A_{3}$ sum to $J_{16,16}-I_{16}$. If $\{i, j, \ell\}=\{1,2,3\}$ then $A_{i}^{2}=5 I_{16}+2\left(A_{j}+A_{\ell}\right)$ and $\left(I_{16}+A_{i}\right)\left(I_{16}+A_{j}\right)=3 J_{16,16}-2\left(I_{16}+A_{\ell}\right)$. The elements of each of $\mathcal{P}, \mathcal{Q}_{u x}, \mathcal{Q}_{u w}$ and $\mathcal{Q}_{x w}$ can be labelled by the same set of size 16 in such a way that the three incidence matrices involving any one are $I_{16}+A_{1}, I_{16}+A_{2}$ and $I_{16}+A_{3}$. Each of these strongly regular graphs contains no triangles, and so together they give a 3-colouring of the edges of $K_{16}$ with no monochromatic triangles. This shows that the corresponding Ramsey number is at least 17, as was first observed by Greenwood and Gleason in [84].

There is more! Replacing the 0 entries of $A_{i}$ by 1 and the 1 entries by -1 gives a Hadamard matrix $H_{i}$, and $H_{i} H_{j}=4 H_{\ell}$. Therefore $\left\{I_{16}, 4^{-1} H_{1}, 4^{-1} H_{2}, 4^{-1} H_{3}\right\}$ forms an elementary Abelian 2-group. Higher even powers of 2 give a similar group formed from Hadamard matrices, and these lead to the multi-stage Youden rectangles in [51] and to the results in [49].

\subsection{Multi-layered Youden rectangles}

An $n \times m$ double Youden rectangle has two sets, $\mathcal{F}$ and $\mathcal{G}$, of partitions of $\Omega$. Those in $\mathcal{F}$ have $m$ parts of size $n$, while those in $\mathcal{G}$ have $n$ parts of size $m$. If $F \in \mathcal{F}$ and $G \in \mathcal{G}$ then $F \perp G$. Each of $\mathcal{F}$ and $\mathcal{G}$ has universal balance, and $|\mathcal{F}|=|\mathcal{G}|=2$. Choose the labelling so that $n<m$.

Multi-stage Youden rectangles satisfy all the same conditions, except that $|\mathcal{F}| \geq 2$ and $|\mathcal{G}|=1$. When $|\mathcal{F}|=2$ this is just a Youden square.

Preece introduced triple Youden rectangles in [163]. The naive reader might think that these would be like double Youden rectangles with $|\mathcal{F}|=$ $|\mathcal{G}|=3$. In fact, they have $|\mathcal{F}|=2$ and $|\mathcal{G}|=3$. He gave examples of size $4 \times 13$ and $7 \times 15$.

Preece and Morgan generalized this concept in [168] by allowing the size of $\mathcal{G}$ to vary. They changed the name to multi-layered Youden rectangle. Thus the partitions in $\mathcal{F}$ are regarded as stages, while those in $\mathcal{G}$ are regarded as layers.

Definition An $n \times m$ multi-layered Youden rectangle with $s$ layers has two sets, $\mathcal{F}$ and $\mathcal{G}$, of partitions of a set $\Omega$ of size $\mathrm{nm}$. Each partition in $\mathcal{F}$ has $m$ parts of size $n$, and each partition in $\mathcal{G}$ has $n$ parts of size $m$, where $n<m$. If $F \in \mathcal{F}$ and $G \in \mathcal{G}$ then $F \perp G$. Each of $\mathcal{F}$ and $\mathcal{G}$ has universal balance, $|\mathcal{F}|=2$ and $|\mathcal{G}|=s$. 
If $s=1$ this is just a Youden square; if $s=2$ it is a double Youden rectangle.

Because $|\mathcal{F}|=2$, a multi-layered Youden rectangle can be shown as an $m \times m$ square with $m-n$ empty cells in each row and column, as in Figure 26(b). Each non-empty cell has $s$ letters, one for each layer. When $s=3$, these are called triple Youden arrays, not to be confused with the triple arrays in Section 13.

In fact, the definitions in $[163,168]$ do not specify universal balance. For $\mathcal{F}$, this is equivalent to pairwise balance, because $|\mathcal{F}|=2$. In all the examples in [163,168], $m-1$ is a multiple of $n$. Therefore pairwise balance among the layers implies that they can all have their parts labelled by the same set in such a way that each incidence matrix between layers is equal to $I_{n}+[(m-1) / n] J_{n n}$. Thus universal balance is guaranteed.

Preece and Morgan give an explicit construction which proves the following.

Theorem 14.6 If $q$ is an odd prime power, $m=2 q+1$ and $s \leq q-4$ then there exists a $q \times(2 q+1)$ multi-layered Youden rectangle with s layers.

\section{Resolved designs}

So far, all designs have been described as collections of partitions of the set $\Omega$ of experimental units. Now we change that viewpoint.

Statisticians usually call each tuple of values of the non-inherent partitions a treatment. Denote by $\Gamma$ the set of treatments.

Definition A design is resolved is there is a partition $D$ of $\Omega$ such that every inherent non-trivial partition $H$ of $\Omega$ satisfies $H \preccurlyeq D$ and every part of $D$ contains each treatment exactly once.

In a resolved design, it is useful to look at the partitions of $\Gamma$ induced within each part of $D$. In the next two subsections we do this for block designs and for row-column designs.

\subsection{Block designs}

Let $B$ be the partition of $\Omega$ into blocks in a resolved block design. For $i=1, \ldots, n_{D}$, let $B_{i}$ be the partition of $\Gamma$ induced by $B$ in the $i$-th part of $D$. Properties of $\left\{B_{1}, \ldots, B_{n_{D}}\right\}$ give information about the block design.

Among the earliest examples of incomplete-block designs were the square lattice designs of Yates [227]. In these, $|\Gamma|=k_{B}^{2}$ and $B_{1}, B_{2}$, 
$\ldots, B_{n_{D}}$ form the partitions of a $k_{B} \times k_{B}$ square into rows, columns and the letters of $n_{D}-2$ mutually orthogonal Latin squares. The design in Figure 2 has this form, as do those made from it by removing one or two parts of $D$.

In a square lattice design, the set of partitions $\left\{B_{1}, \ldots, B_{n_{D}}\right\}$ forms an orthogonal array of strength two on $\Gamma$, with all parts of the same size. In [47], Bose defined affine-resolvable designs. Orthogonal arrays had not yet been invented, but now we can say that affine-resolved designs are precisely those resolved incomplete-block designs for which $\left\{B_{1}, \ldots, B_{n_{D}}\right\}$ forms an orthogonal array of strength two on $\Gamma$ with all parts of the same size.

For example, the first four rows of the orthogonal array in Figure 7 give an affine-resolved incomplete-block design for 12 treatments in eight blocks of size six. More generally, if any row of a Hadamard matrix is normalized to have all its entries the same, then the remaining rows form an orthogonal array of strength two. If an affine-resolved design is made by using each of these rows once, then the block design is balanced in all the senses described in Section 5. Such designs are sometimes called Hadamard 3-designs: see [52, 96].

Many constructions of affine-resolved incomplete-block designs appear in [38], where it is proved that they are optimal among resolved designs.

If $k_{B}^{2}$ does not divide $|\Gamma|$ then an affine-resolvable design is not possible. In work [133] presented at the $1975 \mathrm{BCC}$ in Aberdeen, Patterson and Williams observed that if $n_{D}=2$ then $\left\{B_{1}, B_{2}\right\}$ may be regarded as the partitions $B$ and $L$ of an incomplete-block design with $n_{B}=n_{L}$. For example, the partitions $C$ and $L$ of the set of size 28 in Figure 3 can be used to give a resolved incomplete-block design for 28 treatments in two districts of seven blocks of size four. It was proved in [221] that the resolved design is optimal among resolved designs if and only if the symmetric incomplete-block design is optimal among IBDs of that size. This result gives a technique for finding other optimal resolved incompleteblock designs when $n_{D}=2$ : see [222].

If $n_{D} \geq 3$ and $k_{B}^{2}$ does not divide $|\Gamma|$ then we run into the problem discussed in Section 9: the pairwise relations among $B_{1}, B_{2}, \ldots, B_{n_{D}}$ do not suffice to give the overall properties of the design. Sets of partitions with universal balance have been recommended. When $|\Gamma|=k_{B}\left(k_{B}+1\right)$, the construction in Section 14.2.1 gives resolved IBDs called rectangular lattice designs: see [41, 62, 90, 91, 220].

For other values of $|\Gamma|$, it may be possible to use a set of $n_{D}$ partitions having a different type of universal balance. When $n_{D}=3$ these give the families of resolved incomplete-block designs in [27]. 


\subsection{Nested row-column designs}

In a nested row-column design each part of $D$ is a rectangle with partitions $R$ and $C$ into rows and columns, and $R \wedge C=E$. If it is resolved then $|\Gamma|=k_{R} k_{C}$ and we may consider the partitions $R_{1}, R_{2}, \ldots$, $R_{n_{D}}$ and $C_{1}, C_{2}, \ldots, C_{n_{D}}$ induced on $\Gamma$. Then $R_{i} \perp C_{i}$ for $i=1, \ldots, n_{D}$.

If $k_{R}=k_{C}$ and $n_{D}$ is small enough then we may be able to arrange for all of these partitions to be pairwise strictly orthogonal by using the rows, columns and letters of $2\left(n_{D}-1\right)$ mutually orthogonal $k_{R} \times k_{R}$ Latin squares. These were called quasi-Latin squares when introduced by Yates in [228] but the name soon changed to lattice square designs in [229].

Otherwise, we may be able to choose the partitions so that $R_{i} \perp C_{j}$, $R_{i} \bowtie R_{j}$ and $C_{i} \bowtie C_{j}$ for $\{i, j\} \subseteq\left\{1, \ldots, n_{D}\right\}$ with $i \neq j$. When $n_{D}=2$ this gives a different use for double Youden rectangles, as explained in [39], where it is proved that these are optimal among resolved nested row-column designs. When $n_{D} \geq 3$ we need to consider the multi-way relations among $\left\{R_{1}, \ldots, R_{n_{D}}\right\}$ and $\left\{C_{1}, \ldots, C_{n_{D}}\right\}$, so some of the designs in Section 14.3 may be suitable.

\section{Factorial designs in blocks}

An experiment is called factorial if more than one partition is of interest and can have its parts allocated by the experimenter, as in Example 1.6 and Section 7.2.2. If the only inherent partition is the partition $B$ into blocks, and there are two treatment factors $F$ and $G$, then it may be useful to consider the set $\Delta$ of all triples of values of $F, G$ and $B$ that occur. If we show the parts of $F$ and $G$ as rows and columns, then usually $F \wedge G \neq E$, unlike in classical row-column designs.

Here we give two examples, considering $B, F$ and $G$ as partitions of $\Delta$, to show the effect of different practical constraints.

Example 16.1 Modify Example 1.6 so that there are still six varieties of lettuce and five watering regimes, but now the ten gardens each have room for six vegetable patches and there are no practical constraints. Using a resolved design, as in Section 15.1, it is natural to think of the watering regimes and lettuce varieties as the rows and columns of a $5 \times 6$ rectangle, with five of the blocks corresponding to letters of a Latin square with the last column repeated, and the remaining five blocks corresponding to the letters of a second Latin square, orthogonal to the first, also with the last column repeated. This can give the design in Figure 37.

Here $\Delta$ is the same as $\Omega$. The design has $F \perp G, B \perp G$, and $F \triangleright B$. Thus it is of type C.5 from Section 10.4. 


\begin{tabular}{|cc|cc|cc|cc|cc|cc|}
\hline$A$ & $F$ & $B$ & $G$ & $C$ & $H$ & $D$ & $I$ & $E$ & $J$ & $E$ & $J$ \\
\hline$E$ & $I$ & $A$ & $J$ & $B$ & $F$ & $C$ & $G$ & $D$ & $H$ & $D$ & $H$ \\
\hline$D$ & $G$ & $E$ & $H$ & $A$ & $I$ & $B$ & $J$ & $C$ & $F$ & $C$ & $F$ \\
\hline$C$ & $J$ & $D$ & $F$ & $E$ & $G$ & $A$ & $H$ & $B$ & $I$ & $B$ & $I$ \\
\hline$B$ & $H$ & $C$ & $I$ & $D$ & $J$ & $E$ & $F$ & $A$ & $G$ & $A$ & $G$ \\
\hline
\end{tabular}

Figure 37: Design in Example 16.1: rows represent watering regimes, columns represent lettuce varieties, letters $A-E$ represent the first five blocks and letters $F-J$ represent the other five blocks

\begin{tabular}{|ll|ll|ll|ll|ll|ll|}
\hline$A$ & $B$ & $A$ & $F$ & $A$ & $I$ & $F$ & $I$ & $B$ & $F$ & $B$ & $I$ \\
\hline$B$ & $C$ & $G$ & $J$ & $C$ & $G$ & $C$ & $J$ & $B$ & $G$ & $B$ & $J$ \\
\hline$C$ & $D$ & $D$ & $F$ & $C$ & $H$ & $C$ & $F$ & $F$ & $H$ & $D$ & $H$ \\
\hline$D$ & $E$ & $D$ & $G$ & $G$ & $I$ & $E$ & $I$ & $E$ & $G$ & $D$ & $I$ \\
\hline$A$ & $E$ & $A$ & $J$ & $A$ & $H$ & $E$ & $J$ & $E$ & $H$ & $H$ & $J$ \\
\hline
\end{tabular}

Figure 38: Design in Example 16.2: rows represent drugs, columns represent types of cancer and letters represent medical centres (blocks)

Example 16.2 Valerii Fedorov ${ }^{5}$ posed the problem of assigning cancer types $(C)$ and cancer drugs $(R)$ to medical centres $(B)$ in such a way that no centre deals with very many types of drug or cancer, each centre allocates drugs to patients in such a way that each of its drugs is tested on each of its types of cancer, cancer types and drugs are both balanced with respect to medical centres, and each drug is paired with each cancer type at the same number of medical centres. Since we cannot foresee how many patients will be included in the trial, it make sense to consider the set $\Delta$ of all triples of values of $R, C$ and $B$ that occur.

Figure 38 shows such a design for five drugs, six cancer types and ten medical centres: rows represent drugs, columns represent cancer types, and letters represent medical centres (also called 'blocks'). It was made by assigning a subset of two drugs and a subset of three cancer types to each medical centre, and then giving each medical centre all six combinations of its assigned drugs and cancer types. This method of construction ensures that $R$ and $C$ have adjusted orthogonality with respect to blocks, in addition to the requirements $R \perp C, R \triangleright B$ and $C \triangleright B$. However, the relationships between $R$ and $B$, and $C$ and $B$, are not generalized binary, so the balance is not strict. More designs for this problem are in [34].

${ }^{5}$ Meeting at the Isaac Newton Institute in July 2015 


\section{Some open problems}

1. Divide the cases in list $\mathrm{E}$ in Section 10.6 as finely as those in the other lists in Section 10. Prove that the resulting list gives all possibilities, and give an example of each.

2. Is there an interesting connnection, using finite projective geometry or group theory, between the double Youden rectangle in Figure 30 and the non-extremal triple array in Figure 33?

3. Prove Conjecture 13.5 or find a counter-example.

4. The (hopefully) more unified approach to adjusted balance presented in Sections 9 and 14 is new, so may have errors or things that can be done better. Improve it and take it further.

5. Are there any other families of square matrices, apart from those given in Section 14.2, which can occur as the incidence matrices of a set of partitions with universal balance?

6. Are there designs like those in Sections 14.3-14.4 in which both $|\mathcal{F}|$ and $|\mathcal{G}|$ are greater than two?

Acknowledgements I am very grateful to Donald Preece (R.I.P.) for many illuminating conversations about the topics in this paper over many years. I also thank Chris Brien, Peter Cameron, John Eccleston, Ulrike Grömping, Jonathan Hall, Tomas Nilson, Gordon Royle, Ken Russell and Jennifer Seberry for their very helpful inputs while I was writing this paper.

\section{References}

[1] R. Julian R. Abel, Malcolm Grieg \& D. H. Rees, Existence of OBIBDs with $k=4$ with and without nesting, Discrete Mathematics 266 (2003), 3-36.

[2] K. Afsarinejad \& A. Hedayat, Some contributions to the theory of multistage Youden design, Annals of Statistics 3 (1975), 707-711.

[3] Hira Lal Agrawal, Two way elimination of heterogeneity, Calcutta Statistical Association Bulletin 15 (1966), 32-38.

[4] Hira Lal Agrawal, Some generalizations of distinct representatives with applications to statistical design, Annals of Mathematical Statistics 37 (1966), 525-528. 
[5] Hira Lal Agrawal, Some systematic methods of construction for twoway elimination of heterogeneity, Calcutta Statistical Association Bulletin 15 (1966), 93-108.

[6] Hira Lal Agrawal, Some methods of construction of designs for twoway elimination of heterogeneity-1, Journal of the American Statistical Association 61 (1966), 1153-1171.

[7] H. L. Agrawal \& R. I. Mishra, Some methods of constructing 4DIB designs, Calcutta Statistical Association Bulletin 20 (1971), 89-92.

[8] H. L. Agrawal \& J. Prasad, Some methods of construction of balanced incomplete block designs with nested rows and columns, Biometrika 69 (1982), 481-483.

[9] H. L. Agrawal \& J. Prasad, On construction of balanced incomplete block designs with nested rows and columns, Sankhyā, Series B 45 (1983), 345-350.

[10] H. L. Agrawal \& K. L. Sharma, On construction of two-way designs, Journal of the Indian Statistical Association 13 (1975), 1-31.

[11] H. L. Agrawal \& K. L. Sharma, On construction of four-dimensional incomplete block designs, Journal of the American Statistical Association 73 (1978), 844-849.

[12] D. A. Anderson \& J. A. Eccleston, On the construction of a class of efficient row-column designs, Journal of Statistical Planning and Inference 11 (1985), 131-134.

[13] D. A. Anderson \& W. T. Federer, Multidimensional balanced designs, Communications in Statistics - Theory and Methods 5 (1976), 1193-1204.

[14] I. Anderson, A hundred years of whist tournaments, Journal of Combinatorial Mathematics and Combinatorial Computation 19 (1995), 129-150.

[15] A. Ash, Generalized Youden designs: constructions and tables, Journal of Statistical Planning and Inference 5 (1981), 1-25.

[16] E. F. Assmus, Jr, On the theory of designs, in Surveys in Combinatorics, 1989 (ed. J. Siemons), London Mathematical Society Lecture Notes Series, 141, Cambridge University Press, Cambridge (1989), pp. 1-21. 
[17] Sunanda Bagchi, An infinite series of adjusted orthogonal designs with replication two, Statistica Sinica 6 (1996), 975-987.

[18] Sunanda Bagchi, On two-way designs, Graphs and Combinatorics 14 (1998), 313-319.

[19] S. Bagchi \& E. E. M. van Berkum, On the optimality of a new class of adjusted orthogonal designs, Journal of Statistical Planning and Inference $\mathbf{2 8}$ (1991), 61-65.

[20] Sunanda Bagchi, A. C. Mukhopadhyay \& Bikas K. Sinha, A search for optimal nested row-column designs, Sankhy $\bar{a}$, Series B $\mathbf{5 2}$ (1990), 93-104.

[21] S. Bagchi \& K. R. Shah, On the optimality of a class of row-column designs, Journal of Statistical Planning and Inference 23 (1989), 397-402.

[22] R. A. Bailey, Designs: mappings between structured sets, in Surveys in Combinatorics, 1989 (ed. J. Siemons), London Mathematical Society Lecture Notes Series, 141, Cambridge University Press, Cambridge (1989), pp. 22-51.

[23] R. A. Bailey, Orthogonal partitions in designed experiments, Designs, Codes and Cryptography 8 (1996), 45-77.

[24] R. A. Bailey, Choosing designs for nested blocks, Listy Biometrryczne 36 (1999), 85-126.

[25] R. A. Bailey, Resolved designs viewed as sets of partitions, in Combinatorial designs and their applications (eds. Fred C. Holroyd, Kathleen A. S. Quinn, Chris Rowley \& Bridget S. Webb), Research Notes in Mathematics, 403, Chapman \& Hall, Boca Raton (1999), pp. 1747.

[26] R. A. Bailey, Association Schemes: Designed Experiments, Algebra and Combinatorics, Cambridge Studies in Advanced Mathematics, 84, Cambridge University Press, Cambridge (2004).

[27] R. A. Bailey, Six families of efficient resolvable designs in three replicates, Metrika 62 (2005), 161-173.

[28] R. A. Bailey, Design of Comparative Experiments, Cambridge Series in Statistical and Probabilistic Mathematics, Cambridge University Press, Cambridge (2008). 
[29] R. A. Bailey, Donald Arthur Preece: A life in statistics, mathematics and music, arXiv 1402.2220.

[30] R. A. Bailey, Structures defined by factors, in Handbook of Design and Analysis of Experiments (eds. Angela Dean, Max Morris, John Stufken \& Derek Bingham), Chapman and Hall/ CRC Handbooks of Modern Statistical Methods, Chapman and Hall/ CRC, Boca Raton (2015), pp. 371-414.

[31] R. A. Bailey \& C. J. Brien, Randomization-based models for multitiered experiments: I. A chain of randomizations, Annals of Statistics 44 (2016), 1131-1164.

[32] R. A. Bailey \& Peter J. Cameron, What is a design? How should we classify them?, Designs, Codes and Cryptography 44 (2007), 223238.

[33] R. A. Bailey \& Peter J. Cameron, Combinatorics of optimal designs, in Surveys in Combinatorics 2009 (eds. Sophie Huczynska, James D. Mitchell \& Colva Roney-Dougal), London Mathematical Society Lecture Note Series, 365, (2009), pp. 19-73.

[34] R. A. Bailey \& Peter J. Cameron, Designs which allow each medical centre to treat only a limited number of cancer types with only a limited number of drugs, preprint, University of St Andrews, 2016.

[35] R. A. Bailey, Sandra S. Ferreira, Dário Ferreia \& Célia Nunes, Estimability of variance components when all model matrices commute, Linear Algebra and its Applications 492 (2016), 144-160.

[36] R. A. Bailey, D. C. Goldrei \& D. F. Holt, Block designs with block size two, Journal of Statistical Planning and Inference 10 (1984), 257-263.

[37] R. A. Bailey \& P. Heidtmann, Extremal row-column designs with maximal balance and adjusted orthogonality, preprint, Goldsmiths' College, University of London, 1994.

[38] R. A. Bailey, H. Monod \& J. P. Morgan, Construction and optimality of affine-resolvable designs, Biometrika 82 (1995), 187-200.

[39] R. A. Bailey \& H. D. Patterson, A note on the construction of rowand-column designs with two replicates, Journal of the Royal Statistical Society, Series B 53 (1991), 645-648. 
[40] R. A. Bailey, D. A. Preece \& C. A. Rowley, Randomization for a balanced superimposition of one Youden square on another, Journal of the Royal Statistical Society, Series B 57 (1995), 459-469.

[41] R. A. Bailey \& T. P. Speed, Rectangular lattice designs: efficiency factors and analysis, Annals of Statistics 14 (1986), 874-895.

[42] Simon T. Bate \& Marion J. Chatfield, Identifying the structure of the experimental design, Journal of Quality Technology 48 (2016), 343-364.

[43] Simon T. Bate \& Marion J. Chatfield, Using the structure of the experimental design and the randomization to construct a mixed model, Journal of Quality Technology 48 (2016), 365-387.

[44] Gerhard Behrendt, Equivalence systems with finitely many relations, Monatshefte für Mathematik 103 (1987), 77-83.

[45] Thomas Beth, Dieter Jungnickel \& Hanfried Lenz, Design Theory, Volume I, second edition, Encyclopedia of Mathematics and its Applications, 69, Cambridge University Press, Cambridge (1999).

[46] R. C. Bose, On the construction of balanced incomplete block designs, Annals of Eugenics 9 (1939), 353-399.

[47] R. C. Bose, A note on the resolvability of balanced incomplete block designs, Sankhā 6 (1942), 105-110.

[48] Wayne Broughton, Admissible parameters of symmetric designs satisfying $v=4(k-\lambda)+2$ and symmetric designs with inner balance, Designs, Codes and Cryptography 73 (2014), 77-83.

[49] A. R. Calderbank, P. J. Cameron, W. M. Kantor \& J. J. Seidel, $\mathbb{Z}_{4}$-Kerdock codes, orthogonal spreads, and extremal Euclidean linesets, Proceedings of the London Mathematical Society, Series $3 \mathbf{7 5}$ (1997), 436-480.

[50] Peter J. Cameron, On groups with several doubly-transitive permutation representations, Mathematische Zeitschrift 128 (1972), 1-14.

[51] P. J. Cameron, Multi-letter Youden rectangles from quadratic forms, Discrete Mathematics 266 (2003), 143-151.

[52] P. J. Cameron \& J. H. van Lint, Designs, Graphs, Codes and their Links, London Mathematical Society Student Texts, 22, Cambridge University Press, Cambridge (1991). 
[53] P. J. Cameron \& J. J. Seidel, Quadratic forms over GF(2), Proceedings of the Koninklijke Nederlandse Akademie van Wetenschappen, Series A 76 (1973), 1-8.

[54] B. D. Causey, Some examples of multi-dimensional incomplete block designs, Annals of Mathematical Statistics 39 (1968), 1577-1590.

[55] J. Y. Chang \& W. I. Notz, A method for constructing universally optimal block designs with nested rows and columns, Utilitas Mathematica 38 (1990), 263-276.

[56] C.-S. Cheng, A method for constructing balanced incomplete-block designs with nested rows and columns, Biometrika 73 (1986), 695700 .

[57] C. Christofi, Enumerating $4 \times 5$ and $5 \times 6$ double Youden rectangles, Discrete Mathematics 125 (1994), 129-135.

[58] C. Christofi, On the number of $6 \times 7$ double Youden rectangles, Ars Combinatoria 47 (1997), 223-241.

[59] G. M. Clarke, A second set of treatments in a Youden square design, Biometrics 19 (1963), 98-104.

[60] G. M. Clarke, Four-way balanced designs based on Youden squares with 5, 6, or 7 treatments, Biometrics 23 (1967), 803-812.

[61] Dominique Collombier, Plans d'expérience factoriels, Mathématiques \& Applications, 21, Springer-Verlag, Berlin (1996).

[62] L. C. A. Corsten, Rectangular lattices revisited, in Linear Statistical Inference (eds. T. Caliński \& W. Klonecki), Lecture Notes in Statistics, 35, Springer, Berlin (1985), pp. 29-38.

[63] G. Dall'Aglio, Blocs incomplets éqilibrés orthogonaux, Colloques Internationaux du Centre National de la Recherche Scientifique 110 (1963), 105-214.

[64] A. Dey, U. S. Das \& A. K. Banerjee, Construction of nested balanced incomplete block designs, Calcutta Statistical Association Bulletin 35 (1986), 161-167.

[65] Jean Doyen, Designs and automorphism groups, in Surveys in Combinatorics, 1989 (ed. J. Siemons), London Mathematical Society Lecture Notes Series, 141, Cambridge University Press, Cambridge (1989), pp. 75-83. 
[66] V. Duquenne, What can lattices do for experimental designs?, Mathematical Social Sciences 11 (1986), 243-281.

[67] Thomas Durt, Berthold-Georg Englert, Ingemar Bengtsson \& Karol Życzkowski, On mutually unbiased bases, International Journal of Quantum Information 8 (2010), 535-640.

[68] J. A. Eccleston \& J. A. John, Recovery of row and column information in row-column designs with adjusted orthogonality, Journal of the Royal Statistical Society, Series B 48 (1986), 238-243.

[69] J. A. Eccleston, J. A. John \& D. Whitaker, Some row-column designs with adjusted orthogonality, Journal of Statistical Planning and Inference 36 (1993), 323-330.

[70] J. A. Eccleston \& J. Kiefer, Relationships of optimality for individual factors of a design, Journal of Statistical Planning and Inference $\mathbf{5}$ (1981), 213-219.

[71] J. A. Eccleston \& C. A. McGilchrist, Algebra of a row-column design, Journal of Statistical Planning and Inference 12 (1985), 305310 .

[72] J. Eccleston \& K. Russell, Connectedness and orthogonality in multifactor designs, Biometrika 62 (1975), 341-345.

[73] J. A. Eccleston \& K. G. Russell, Adjusted orthogonality in nonorthogonal designs, Biometrika 64 (1977), 339-345.

[74] J. A. Eccleston \& A. Street, Construction for adjusted orthogonal designs, Ars Combinatoria 28 (1990), 117-128.

[75] W. L. Edge, The geometry of the linear fractional group $\operatorname{LF}(4,2)$, Proceedings of the London Mathematical Society, Series 34 (1954), 317-342.

[76] P. Eendebak \& E. Schoen, Complete series of non-isomorphic orthogonal arrays, http://pietereendebak.nl/oapage/

[77] D. J. Finney, The fractional replication of factorial experiments, Annals of Eugenics 12 (1945), 291-301.

[78] R. A. Fisher, Design of Experiments, Oliver and Boyd, Edinburgh (1935).

[79] R. A. Fisher, An examination of the different possible solutions of a problem in incomplete blocks, Annals of Eugenics 10 (1940), 52-75. 
[80] R. A. Fisher \& F. Yates, Statistical Tables for Biological, Agricultural and Medical Research, Oliver and Boyd, Edinburgh (1938).

[81] Dmitri G. Fon-Der-Flaass, Arrays of distinct representatives a very simple NP-complete problem, Discrete Mathematics 171 (1997), 295-298.

[82] G. H. Freeman, Some non-orthogonal partitions of $4 \times 4,5 \times 5$ and $6 \times 6$ Latin squares, Annals of Mathematical Statistics 37 (1966), 666-681.

[83] Dennis C. Gilliland, A note on orthogonal partitions and some wellknown structures in design of experiments, Annals of Statistics 5 (1977), 565-570.

[84] R. E. Greenwood \& A. M. Gleason, Combinatorial relations and chromatic graphs, Canadian Journal of Mathematics 7 (1955), 1-7.

[85] M. Greig \& D. H. Rees, Existence of balanced incomplete block designs for many sets of treatments, Discrete Mathematics 261 (2003), 299-324.

[86] Ulrike Grömping \& R. A. Bailey, Regular fractions of factorial arrays, in mODa 11 -Advances in Model-Oriented Design and Analysis (eds. Joachim Kunert, Christine H. Müller \& Anthony C. Atkinson), Springer International Publishing, Switzerland (2016), pp. 143-151.

[87] Heiko Großmann, Automating the analysis of variance of orthogonal designs, Computational Statistics and Data Analysis 70 (2014), 118.

[88] J. I. Hall, On identifying $\mathrm{PG}(3,2)$ and the complete 3-design on seven points, Annals of Discrete Mathematics 7 (1980), 131-141.

[89] P. Hall, On representatives of subsets, Journal of the London Mathematical Society 10 (1935), 26-30.

[90] B. Harshbarger, Preliminary report on the rectangular lattices, Biometrics 2 (1946), 115-119.

[91] B. Harshbarger, Triple rectangular lattices, Biometrics 5 (1949), 113.

[92] A. Hedayat \& W. T. Federer, Pairwise and variance balanced incomplete block designs, Annals of the Institute of Statistical Mathematics 26 (1974), 331-338. 
[93] A. Hedayat, E. T. Parker \& W. T. Federer, The existence and construction of two families of designs for two successive experiments, Biometrika 57 (1970), 351-355.

[94] A. Hedayat, E. Seiden \& W. T. Federer, Some families of designs for multistage experiments: mutually balanced Youden designs when the number of treatments is a prime power or twin primes. I, Annals of Mathematical Statistics 43 (1972), 1517-1527.

[95] A. S. Hedayat, N. J. A. Sloan \& J. Stufken, Orthogonal Arrays, Springer-Verlag, New York (1999).

[96] A. Hedayat \& W. D. Wallis, Hadamard matrices and their applications, Annals of Statistics 6 (1978), 1184-1238.

[97] J. W. P. Hirschfeld, Finite Projective Spaces of Three Dimensions, Oxford Mathematical Monographs, Oxford University Press, Oxford (1985).

[98] T. N. Hoblyn, S. C. Pearce \& G. H. Freeman, Some considerations in the design of successive experiments in fruit plantations, Biometrics 10 (1954), 503-515.

[99] A. T. James, The relationship algebra of an experimental design, Annals of Mathematical Statistics 28 (1957), 993-1002.

[100] A. T. James \& G. N. Wilkinson, Factorization of the residual operator and canonical decomposition of nonorthogonal factors in the analysis of variance, Biometrika 58 (1971), 279-294.

[101] M. Jimbo \& S. Kuriki, Construction of nested designs, Ars Combinatoria 16 (1983), 275-285.

[102] J. A. John \& J. A Eccleston, Row-column $\alpha$-designs, Biometrika 73 (1986), 301-306.

[103] Byron Jones, Algorithms to search for optimal row-and-column designs, Journal of the Royal Statistical Society, Series B 41 (1979), 210-216.

[104] Byron Jones, Algorithm AS156: Combining two component designs to form a row-and-column design, Applied Statistics 29 (1980), 334345 .

[105] R. Morley Jones, On a property of incomplete blocks, Journal of the Royal Statistical Society, Series B 21 (1959), 172-179. 
[106] B. Kassanis \& A. Kleczkowski, Inactivation of a strain of tobacco necrosis virus and of the RNA isolated from it, Photochemistry and Photobiology 4 (1965), 209-214.

[107] O. Kempthorne, G. Zyskind, S. Addelman, T. N. Throckmorton \& R. N. White, Analysis of Variance Procedures, Aeronautical Research Laboratory, Ohio, Report No. 149, 1961.

[108] J. Kiefer, On the nonrandomized optimality and randomized nonoptimality of symmetrical designs, Annals of Mathematical Statistics 29 (1958), 675-699.

[109] J. Kiefer, Balanced block designs and generalized Youden designs, I. Construction (patchwork), Annals of Statistics 3 (1975), 109-118.

[110] K. Kishen, Symmetrical unequal block arrangements, Sankhyā 5 (1940-1941), 329-344.

[111] A. Kleczkowski, Interpreting relationships between the concentrations of plant viruses and numbers of local lesions, Journal of General Microbiology 4 (1950), 53-69.

[112] A. Kobilinsky \& H. Monod, Experimental designs generated by group morphisms, Scandinavian Journal of Statistics 18 (1991), 119-134.

[113] A. M. Kshirsagar, On balancing in designs in which heterogeneity is eliminated in two directions, Calcutta Statistical Assocation Bulletin 7 (1957), 161-166.

[114] Heinz Lüneberg, Transitive Erweiterungen endlicher Permutationsgruppen, Lecture Notes in Mathematics, 4, Springer-Verlag, Berlin (1969).

[115] H. B. Mann, The algebra of a linear hypothesis, Annals of Mathematical Statistics 31 (1960), 1-15.

[116] John P. McSorley, Double arrays, triple arrays, and balanced grids with $v=r+c-1$, Designs, Codes and Cryptography 37 (2005), 313-318.

[117] John P. McSorley \& Nicholas C. Phillips, Complete enumeration and properties of binary pseudo-Youden designs $\operatorname{PYD}(9,6,6)$, Journal of Statistical Planning and Inference 137 (2007), 1464-1473. 
[118] John P. McSorley, N. C. K. Phillips, W. D. Wallis \& J. L. Yucas, Double arrays, triple arrays and balanced grids, Designs, Codes and Cryptography 35 (2005), 21-45.

[119] J. P. Morgan, Nested designs, in Design and Analaysis of Experiments (eds. S. Ghosh \& C. R. Rao), Handbook of Statistics, 13, Elsevier Science, Amsterdam (1996), pp. 939-976.

[120] J. P. Morgan, Properties of superimposed BIBDs, Journal of Statistical Planning and Inference 73 (1998), 135-148.

[121] J. P. Morgan, D. A. Preece \& D. H. Rees, Nested balanced incomplete block designs, Discrete Mathematics 231 (2001), 351-389.

[122] John P. Morgan \& Nizam Uddin, Optimality and construction of nested row and column designs, Journal of Statistical Planning and Inference $\mathbf{3 7}$ (1993), 81-93.

[123] J. P. Morgan \& Nizam Uddin, Optimal blocked main effects plans with nested rows and columns and related designs, Annals of Statistics 24 (1996), 1185-1208.

[124] Rahul Mukerjee \& Sudhir Gupta, Geometric construction of balanced block designs with nested rows and columns, Discrete Mathematics 91 (1991), 105-108.

[125] G. Nageswara Rao, Further contributions to balanced generalized two-way elimination of heterogeneity designs, Sankhyā 38 (1976), $72-79$.

[126] J. A. Nelder, The analysis of randomized experiments with orthogonal block structure. I. Block structure and the null analysis of variance, Proceedings of the Royal Society of London, Series A 283 (1965), 147-162.

[127] J. A. Nelder, The analysis of randomized experiments with orthogonal block structure. II. Treatment structure and the general analysis of variance, Proceedings of the Royal Society of London, Series A 283 (1965), 163-178.

[128] J. A. Nelder, The combination of information in generally balanced designs, Journal of the Royal Statistical Society, Series B 30 (1968), 303-311. 
[129] Tomas Nilson, Row-column designs with adjusted orthogonality, Masters thesis, Mid Sweden University, Department of Engineering, Physics and Mathematics, 2007.

[130] Tomas Nilson \& Peter J. Cameron, Triple arrays from difference sets, arXiv 1609.00152.

[131] Tomas Nilson \& Pia Heidtmann, Inner balance of symmetric designs, Designs, Codes and Cryptography 71 (2014), 247-260.

[132] Tomas Nilson \& Lars-Daniel Öhman, Triple arrays and Youden squares, Designs, Codes and Cryptography 75 (2015), 429-451.

[133] H. D. Patterson \& E. R. Williams, Some theoretical results on general block designs, Congressus Numerantium 15 (1976), 489-496.

[134] S. C. Pearce, The use and classification of non-orthogonal designs, Journal of the Royal Statistical Society, Series A 126 (1963), 353377.

[135] S. C. Pearce, The efficiency of block designs in general, Biometrika 57 (1970), 339-346.

[136] S. C. Pearce \& J. Taylor, The changing of treatments in a long-term trial, Journal of Agricultural Science 38 (1948), 402-410.

[137] N. C. K. Phillips \& D. A. Preece, Finding double Youden rectangles, in Designs 2002: Further Computational and Constructive Design Theory (ed. W. D. Wallis), Mathematics and its Applications, 563, Kluwer Academic Publishing, Boston MA (2003), pp. 301-315.

[138] N. C. K. Phillips, D. A. Preece \& D. H. Rees, Double Youden rectangles for the four biplanes with $k=9$, Journal of Combinatorial Mathematics and Combinatorial Computing 44 (2003), 169-176.

[139] N. C. K. Phillips, D. A. Preece \& W. D. Wallis, The seven classes of $5 \times 6$ triple arrays, Discrete Mathematics 293 (2005), 213-218.

[140] N. C. K. Phillips \& W. D. Wallis, An elusive array, Bulletin of the Institute of Combinatorics and its Applications 39 (2003), 39-40.

[141] Giovanni Pistone \& Maria-Piera Rogantin, Indicator function and complex coding for mixed fractional factorial designs, Journal of Statistical Planning and Inference 138 (2008), 787-802.

[142] R. L. Plackett \& J. P. Burman, The design of optimum multifactorial experiments, Biometrika 33 (1946), 305-325. 
[143] Richard F. Potthoff, Three-factor additive designs more general than the Latin square, Technometrics 4 (1962), 187-208.

[144] Richard F. Potthoff, Some illustrations of four-dimensional incomplete block constructions, Calcutta Statistical Association Bulletin 12 (1963), 19-30.

[145] D. A. Preece, Some row and column designs for two sets of treatments, Biometrics 22 (1966), 1-25.

[146] D. A. Preece, Classifying Youden rectangles, Journal of the Royal Statistical Society, Series B 28 (1966), 118-130.

[147] D. A. Preece, Some balanced incomplete block designs for two sets of treatments, Biometrika 53 (1966), 497-506.

[148] D. A. Preece, Nested balanced incomplete block designs, Biometrika 54 (1967), 479-486.

[149] D. A. Preece, Balanced $6 \times 6$ designs for 9 treatments, Sankhy $\bar{a}$, Series B 30 (1968), 443-446.

[150] D. A. Preece, Some new balanced row-and-column designs for two non-interacting sets of treatments, Biometrics 27 (1971), 426-430.

[151] D. A. Preece, Non-orthogonal Graeco-Latin designs, in Combinatorial Mathematics IV (eds. Louis R. A. Casse \& Walter D. Wallis), Lecture Notes in Mathematics, 560, Springer-Verlag, Berlin (1976), pp. 7-26.

[152] D. A. Preece, Some designs based on $11 \times 5$ Youden 'squares', Utilitas Mathematica 9 (1976), 139-146.

[153] D. A. Preece, A second domain of balanced $6 \times 6$ designs for nine equally-replicated treatments, Sankhyā, Series B 38 (1976), 192194.

[154] D. A. Preece, Orthogonality and designs: a terminological muddle, Utilitas Mathematica 12 (1977), 201-223.

[155] D. A. Preece, Balance and designs: another terminological tangle, Utilitas Mathematica 21C (1982), 85-186.

[156] D. A. Preece, Some partly cyclic $13 \times 4$ Youden 'squares' and a balanced arrangement for a pack of cards, Utilitas Mathematica 22 (1982), 255-263. 
[157] D. A. Preece, Genstat analyses for complex balanced designs with non-interacting factors, Genstat Newsletter 21 (March 1988), 33-45.

[158] D. A. Preece, Fifty years of Youden squares: a review, Bulletin of the Institute of Mathematics and its Applications 26 (1990), 65-75.

[159] D. A. Preece, Double Youden rectangles of size $6 \times 11$, Mathematical Scientist 16 (1991), 41-45.

[160] D. A. Preece, Enumeration of some $7 \times 15$ Youden squares and construction of some $7 \times 15$ double Youden rectangles, Utilitas Mathematica 41 (1992), 51-62.

[161] D. A. Preece, A set of double Youden rectangles of size $8 \times 15$, Ars Combinatoria 36 (1993), 215-219.

[162] D. A. Preece, Double Youden rectangles - an update with examples of size $5 \times 11$, Discrete Mathematics 125 (1994), 309-317.

[163] D. A. Preece, Triple Youden rectangles: A new class of fully balanced combinatorial arrangements, Ars Combinatoria 37 (1994), 175-182.

[164] D. A. Preece, Multi-factor balanced block designs with complete adjusted orthogonality for all pairs of treatment factors, Australian Journal of Statistics 38 (1996), 223-230.

[165] D. A. Preece, Some $6 \times 11$ Youden squares and double Youden rectangles, Discrete Mathematics 167/168 (1997), 527-541.

[166] D. A. Preece, P. W. Brading, C. W. H. Lam \& M. Côté, Balanced $6 \times 6$ designs for 4 equally replicated treatments, Discrete Mathematics 125 (1994), 319-327.

[167] D. A. Preece \& P. J. Cameron, Some new fully-balanced GraecoLatin Youden 'squares', Utilitas Mathematica 8 (1975), 193-204.

[168] D. A. Preece \& J. P. Morgan, Multi-layered Youden rectangles, Journal of Combinatorial Designs, 25 (2017), 75-84. doi:10.1002/jcd.21252

[169] D. A. Preece \& N. C. K. Phillips, A new type of Freeman-Youden rectangle, Journal of Combinatorial Mathematics and Combinatorial Computing 25 (1997), 65-78.

[170] D. A. Preece, D. H. Rees \& J. P. Morgan, Doubly nested balanced incomplete block designs, Congressus Numerantium 137 (1999), 518. 
[171] D. A. Preece \& B. J. Vowden, Graeco-Latin squares with embedded balanced superpositions of Youden squares, Discrete Mathematics 138 (1995), 353-363.

[172] D. A. Preece, B. J. Vowden \& N. C. K. Phillips, Double Youden rectangles of sizes $p \times(2 p+1)$ and $(p+1) \times(2 p+1)$, Ars Combnatoria 51 (1999), 161-171.

[173] D. A. Preece, B. J. Vowden \& N. C. K. Phillips, Double Youden rectangles of sizes $(p+1) \times\left(p^{2}+p+1\right)$, Utilitas Mathematica 59 (2001), 139-154.

[174] D. A. Preece, W. D. Wallis \& J. L. Yucas, Paley triple arrays, Australasian Journal of Combinatorics 33 (2005), 237-246.

[175] The R Project for Statistical Computing, https://www.r-project.org.

[176] D. Raghavarao, A note on some balanced generalized two-way elimination of heterogeneity designs, Journal of the Indian Society of Agricultural Statistics 22 (1970), 49-52.

[177] D. Raghavarao, Constructions and Combinatorial Problems in Design of Experiments, John Wiley and Sons, New York (1971).

[178] D. Raghavarao \& G. Nageswararao, A note on a method of construction of designs for two-way elimination of heterogeneity, Communications in Statistics 3 (1974), 197-199.

[179] C. R. Rao, Factorial arrangements derivable from combinatorial arrangements of arrays, Journal of the Royal Statistical Society, Supplement 9 (1947), 128-139.

[180] C. R. Rao, On a class of arrangements, Proceedings of the Edinburgh Mathematical Society 8 (1949), 119-125.

[181] D. H. Rees \& D. A. Preece, Perfect Graeco-Latin balanced incomplete block designs (pergolas), Discrete Mathematics 197/198 (1999), 691-712.

[182] K. B. Reid \& E. Brown, Doubly regular tournaments are equivalent to skew Hadamard matrices, Journal of Combinatorial Theory, Series A 12 (1972), 332-338.

[183] Kenneth Graham Russell, On the theory of row-column designs, $\mathrm{PhD}$ thesis, University of New South Wales, 1977. 
[184] K. G. Russell, A comparison of six methods of analysing row-column designs with inter-block information, Statistics and Computing $\mathbf{9}$ (1999), 239-246.

[185] K. G. Russell, J. A. Eccleston \& G. J. Knudsen, Algorithms for the construction of $(M, S)$ - optimal block designs and row-column designs, Journal of Statistical Computation and Simulation 12 (1981), 93-105.

[186] G. M. Saha \& A. D. Das, A note on construction of mutually balanced Youden designs, Utilitas Mathematica 33 (1988), 5-8.

[187] Jennifer Seberry, A note on orthogonal Graeco-Latin designs, Ars Combinatoria 8 (1979), 85-94.

[188] E. Seiden \& C. Y. Wu, A geometric construction of generalized Youden designs for $v$ a power of a prime, Annals of Statistics 6 (1978), 452-460.

[189] K. R. Shah, On uniformly better combined estimates in row-column designs with adjusted orthogonality, Communications in StatisticsTheory and Methods 17 (1988), 3121-3124.

[190] K. R. Shah \& J. A. Eccleston, On some aspects of row-column designs, Journal of Statistical Planning and Inference 15 (1986), 87-95.

[191] Kirti R. Shah \& Bikas K. Sinha, Theory of Optimal Designs, Lecture Notes in Statistics, 54, Springer-Verlag, New York (1989).

[192] S. S. Shrikande, Designs for two-way elimination of heterogeneity, Annals of Mathematical Statistics 22 (1951), 235-247.

[193] M. Singh \& A. Dey, Two-way elimination of heterogeneity, Journal of the Royal Statistical Society, Series B 40 (1978), 58-63.

[194] M. Singh \& A. Dey, Block designs with nested rows and columns, Biometrika 66 (1979), 321-326.

[195] N. P. Singh \& Gular Singh, Analysis of row-column experiments involving several non-interacting-sets of treatments and multistage Youden square designs, Biometrical Journal 26 (1984), 893-899.

[196] C. A. B. Smith \& H. O. Hartley, The construction of Youden squares, Journal of the Royal Statistical Society, Series B 10 (1948), 262-263. 
[197] T. P. Speed \& R. A. Bailey, On a class of association schemes derived from lattices of equivalence relations, in Algebraic Structures and Applications (eds. P. Schulz, C. E. Praeger \& R. P. Sullivan), Marcel Dekker, New York (1982), pp. 55-74.

[198] T. P. Speed \& R. A. Bailey, Factorial dispersion models, International Statistical Review 55 (1987), 261-277.

[199] Edward Spence, Construction and classification of combinatorial designs, in Surveys in Combinatorics, 1995 (ed. Peter Rowlinson), London Mathematical Society Lecture Notes Series, 218, Cambridge University Press, Cambridge (1995), pp. 191-213.

[200] S. K. Srivastav \& J. P. Morgan, On the class of $2 \times 2$ balanced incomplete block designs with nested rows and columns, Communications in Statistics - Theory and Methods 25 (1996), 1859-1870.

[201] Leon S. Sterling \& Nicholas Wormald, A remark on the construction of designs for two-way elimination of heterogeneity, Bulletin of the Australian Mathematical Society 14 (1976), 383-388.

[202] Anne Penfold Street \& Deborah J. Street, Combinatorics of Experimental Design, Oxford University Press, Oxford (1987).

[203] Deborah J. Street, Graeco-Latin and nested row and column designs, in Combinatorial Mathematics VIII (ed. Kevin L. McAvaney), Lecture Notes in Mathematics, 884, Springer-Verlag, Berlin (1981), pp. 304-313.

[204] W. B. Taylor, Incomplete block designs with row balance and recovery of inter-block information, Biometrics 13 (1957), 1-12.

[205] T. N. Throckmorton, Structures of classification data, PhD thesis, Ames, Iowa, 1961.

[206] Tue Tjur, Analysis of variance models in orthogonal designs, International Statistical Review 52 (1984), 33-65.

[207] Tue Tjur, Analysis of variance and design of experiments, Scandinavian Journal of Statistics 18 (1991), 273-322.

[208] K. D. Tocher, The design and analysis of block experiments, Journal of the Royal Statistical Society, Series B 14 (1952), 45-91.

[209] Nizam Uddin, Constructions for some balanced incomplete block designs with nested rows and columns, Journal of Statistical Planning and Inference 31 (1992), 253-261. 
[210] Nizam Uddin, On recursive construction for balanced incomplete block designs with nested rows and columns, Metrika 42 (1995), 341-345.

[211] Nizam Uddin \& John P. Morgan, Some constructions for balanced incomplete block designs with nested rows and columns, Biometrika 77 (1990), 193-202.

[212] Nizam Uddin \& John P. Morgan, Two constructions for balanced incomplete block designs with nested rows and columns, Statistica Sinica 1 (1991), 229-232.

[213] P. M. van de Ven \& A. Di Bucchianico, On the equivalence of definitions for regular fractions of mixed-level factorial designs, Journal of Statistical Planning and Inference 139 (2009), 2351-2361.

[214] Barry Vowden, Infinite series of double Youden rectangles, Discrete Mathematics 125 (1994), 385-391.

[215] B. J. Vowden, A new infinite series of double Youden rectangles, Ars Combinatoria 56 (2000), 133-145.

[216] B. J. Vowden \& D. A. Preece, Some new infinite series of FreemanYouden rectangles, Ars Combinatoria 51 (1999), 49-63.

[217] W. D. Wallis, Triple arrays and related designs, Discrete Applied Mathematics 163 (2014), 220-236.

[218] W. D. Wallis \& J. L. Yucas, Note on Agrawal's "Designs for Two-way Elimination of Heterogeneity", Journal of Combinatorial Mathematics and Combinatorial Computation 46 (2003), 155-160.

[219] E. R. Williams, Efficiency-balanced designs, Biometrika 62 (1975), 686-689.

[220] E. R. Williams, A note on rectangular lattice designs, Biometrics 33 (1977), 410-414.

[221] E. R. Williams, H. D. Patterson \& J. A. John, Resolvable designs with two replications, Journal of the Royal Statistical Society, Series B 38 (1976), 296-301.

[222] E. R. Williams, H. D. Patterson \& J. A. John, Efficient two-replicate resolvable designs, Biometrics 33 (1977), 713-717. 
[223] R. M. Wilson, An existence theorem for pairwise balanced designs. III. Proof of the existence conjecture, Journal of Combinatorial Theory, Series A 18 (1975), 71-79.

[224] F. Yates, Complex experiments, Journal of the Royal Statistical Society, Supplement 2 (1935), 181-247.

[225] F. Yates, Incomplete randomized blocks, Annals of Eugenics 7 (1936), 121-140.

[226] F. Yates, Incomplete Latin squares, Journal of Agricultural Science 26 (1936), 301-315.

[227] F. Yates, A new method of arranging variety trials involving a large number of varieties, Journal of Agricultural Science 26 (1936), 424455 .

[228] F. Yates, A further note on the arrangement of variety trials: quasiLatin squares, Annals of Eugenics 7 (1937), 319-332.

[229] F. Yates, Lattice squares, Journal of Agricultural Science 30 (1940), 672-787.

[230] W. J. Youden, Use of incomplete block replications in estimating tobacco-mosaic virus, Contributions from Boyce Thompson Institute 9 (1937), 41-48.

[231] Joseph L. Yucas, The structure of a $7 \times 15$ triple array, Congressus Numerantium 154 (2002), 43-47.

[232] G. Zyskind, On structure, relation, sigma, and expectation of mean squares, Sankhyā, Series A 24 (1962), 115-148.

School of Mathematics and Statistics,

University of St Andrews,

North Haugh,

St Andrews,

Fife KY16 9SS, U.K.

rab24@st-andrews.ac.uk 